\title{
Monitoring dynamics of protein nascent chain on the ribosome using PET-FCS
}

\author{
Dissertation \\ for the award of the degree \\ "Doctor of Philosophy" \\ of the Georg-August-Universität Göttingen \\ within the doctoral program IMPRS Molecular Biology \\ of the Georg-August University School of Science (GAUSS)
}

submitted by

Marija Liutkute

from Vilnius, Lithuania 
When we try to pick out anything by itself, we find it hitched to everything else in the universe.

John Muir 
Members of Thesis Committee:

Prof. Dr. Marina V. Rodnina ( $1^{\text {st }}$ reviewer)

Department of Physical Biochemistry

Max Planck Institute for Biophysical Chemistry

Prof. Dr. Joerg Enderlein (2 ${ }^{\text {nd }}$ reviewer)

III. Institute of Physics - Biophysics, Faculty of Physics

Georg-August-University Göttingen

Prof. Dr. Kai Tittmann

Department of Bioanalytics, Faculty of Biology and Physiology

Georg-August-University Göttingen

Prof. Dr. Patrick Cramer

Department of Molecular Biology

Max Planck Institute for Biophysical Chemistry

Prof. Dr. Holger Stark

3D-Cryo Electron Microscopy

Max Planck Institute for Biophysical Chemistry

Dr. Alex Faesen

Department of Biochemistry of Signal Dynamics

Max Planck Institute for Biophysical Chemistry

Date of the oral examination: May $20^{\text {th }}, 2020$ 
Affidavit

I hereby declare that I prepared the dissertation "Monitoring dynamics of protein nascent chain on the ribosome using PET-FCS" on my own and with no other sources and aids than quoted.

Marija Liutkute

Göttingen, March 2020 


\section{Related publications}

Liutkute, M., Samatova, E. \& Rodnina, M.V. Cotranslational Folding of Proteins on the Ribosome. Biomolecules 10 (2020). doi: 10.3390/biom10010097 


\section{Table of Contents}

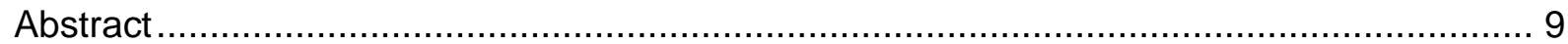

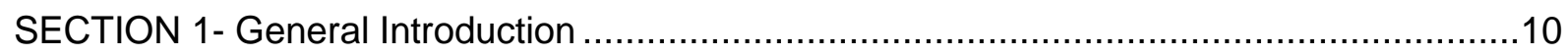

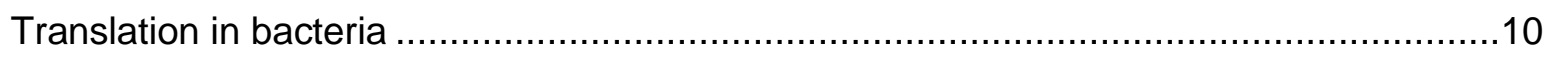

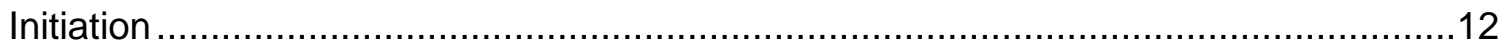

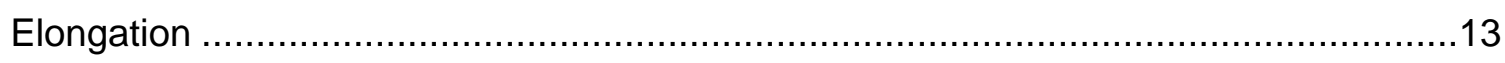

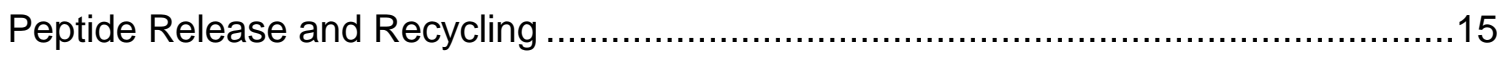

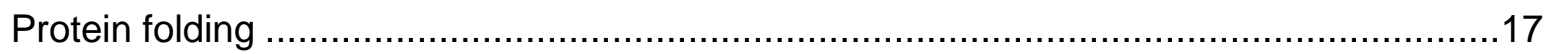

Folding Model: Framework (diffusion collision) ................................................ 18

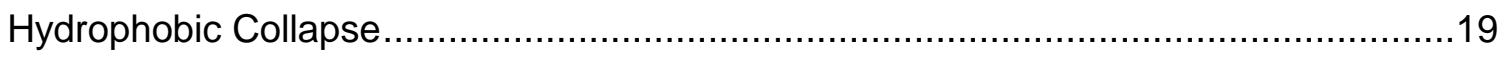

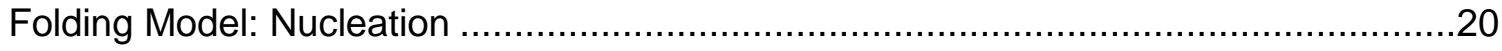

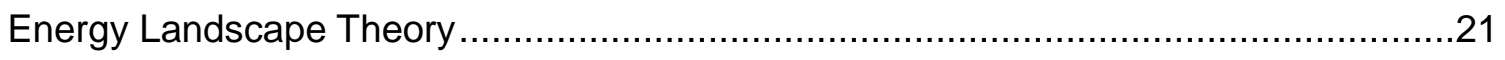

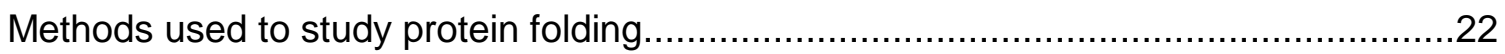

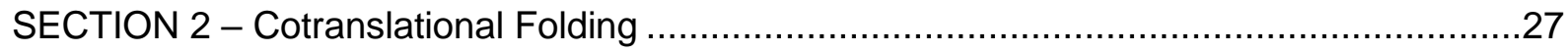

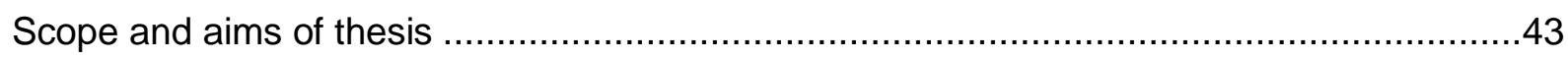

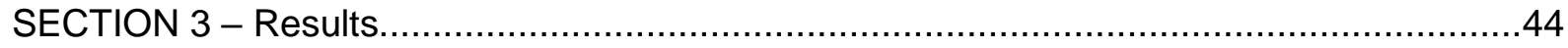

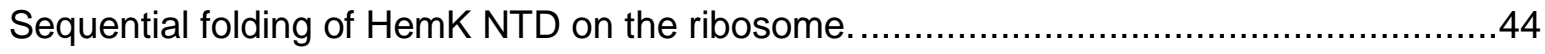

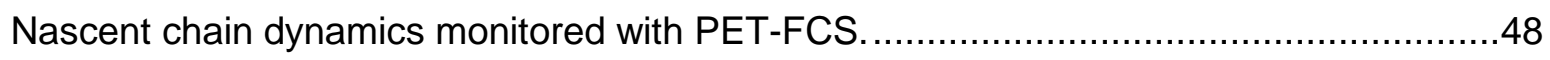

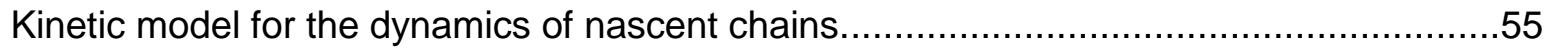

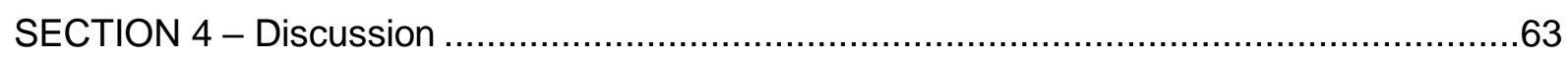

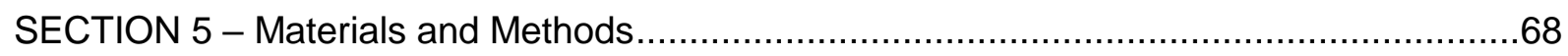

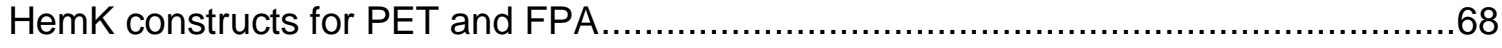

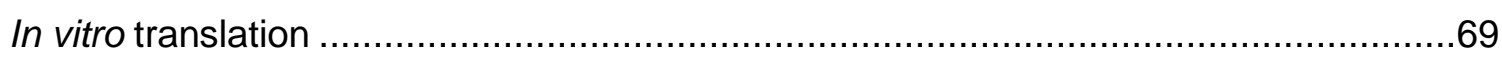

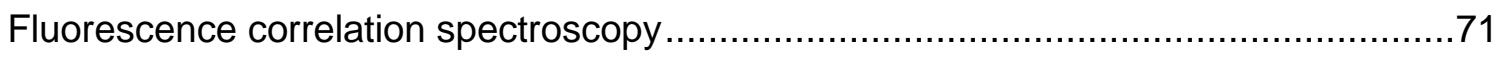

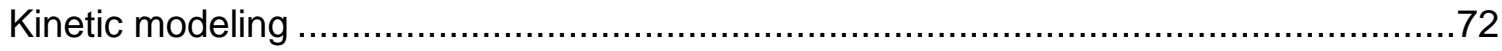

Calculations of the transition state energy barrier .............................................. 73

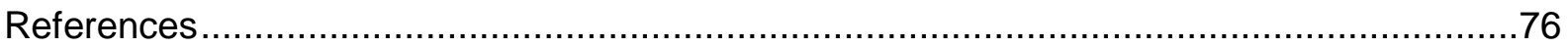

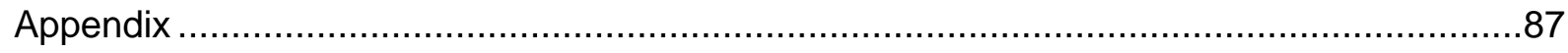

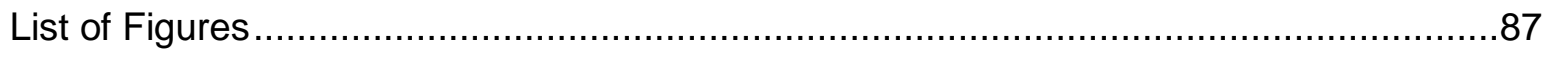

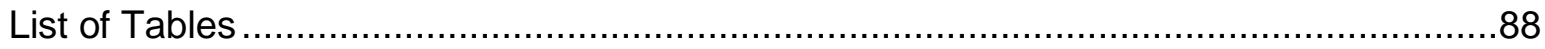


List of Equations.

.88

DNA sequence 5' to 3' of HemK wt FPA constructs...

.89

Acknowledgements

101 


\section{Abstract}

The ribosome synthesizes proteins according to the sequence of the messenger RNA (mRNA) by progressively adding amino acids to the $\mathrm{C}$-terminus of the nascent peptide. For a protein to become fully functional, it has to fold into a specific three-dimensional structure, called the native state. Experimental work on protein folding in solution has shown several possible models of protein folding. However, currently there is no unifying model for protein folding on the ribosome. Recent experimental work has shown that in the cell nascent polypeptides begin to fold during ongoing translation and in the constrained space of the ribosomal peptide exit tunnel. Here we propose to utilize two novel experimental techniques, force profile assay (FPA) and photoinduced electron transfer with fluorescence correlation spectroscopy (PET-FCS) to map the folding trajectory and obtain temporal information on rapid local structural fluctuations of dynamic cotranslational intermediates. We studied how a small $\alpha$-helical domain, the $\mathrm{N}$ terminal domain of HemK, folds cotranslationally using a high-resolution force profile assay. FPA reveals that the protein starts to compact as soon as the $\mathrm{N}$-terminal $\alpha$-helical segments are synthesized. Compaction proceeds vectorially - as nascent chain grows, emerging helical segments dock onto the preceding structures and rearrangements continue after emergence out of the tunnel and near the ribosomal surface. PET-FCS shows that at each stage of translation the nascent peptide undergoes structural fluctuations on the $\mu$ s time scale. As the domain grows in length and the complexity of tertiary interactions and moves away from the ribosome the fluctuations slow down. Native state destabilizing mutations have little effect on the folding pathway inside the ribosome exit tunnel, but disrupt the final domain stabilization. The results presented in this thesis show how FPA and PET-FCS method can be utilized in solving the trajectory of cotranslational protein folding and characterizing the dynamic properties of folding intermediates. 


\section{SECTION 1- General Introduction}

Proteins are a major class of essential biomolecules that carry out many functions in cells. Some perform enzymatic catalysis (kinases, phosphatases, lipases), transport (hemoglobin), storage (casein, ovalbumin), contract muscles (actin and myosin), provide structure (collagen, elastin and keratin), protect/defend the organism (thrombin, antibodies), regulate processes (hormones, transcription factors), and act as receptors (synaptic receptors). All proteins are synthesized within cells by creating a polymer from individual amino acid building blocks, using a messenger RNA (mRNA) as a template in a process called translation.

\section{Translation in bacteria}

Translation is performed by ribosomes, which are macromolecular machines that are conserved throughout the domains or life. A prokaryotic 70S ribosome ( $\mathrm{S}$ - Svedberg unit) is comprised of two subunits consisting of ribosomal RNA (rRNA) and proteins. The large ribosomal subunit (50S) in bacteria is comprised of two rRNA molecules $23 \mathrm{~S}$ and $5 \mathrm{~S}$ as well as 34 different proteins. The small ribosomal subunit (30S) has one rRNA molecule $16 \mathrm{~S}$ and 21 proteins. When not engaged in translation the subunits exist independently, but during the first steps of translation these associate together to become a functional organelle.

The ribosome has three tRNA binding sites - E, P and A. During translation the $A$ site is where the aminocylated tRNAs (aa-tRNA) bind and the $P$ site is where the tRNA with attached nascent chain resides. The $E$ site is where deacylated tRNA is positioned before leaving the ribosome. The decoding center is located on the $30 \mathrm{~S}$ subunit and this is where codon anticodon interactions are formed between the template mRNA and the incoming aa-tRNA. The catalytic center for peptide bond formation between amino acids is located in the $50 \mathrm{~S}$ subunit and is called the peptidyl-transferase center, encompassing the $50 \mathrm{~S}$ parts of the $A$ and $P$ sites (Figure 1). 


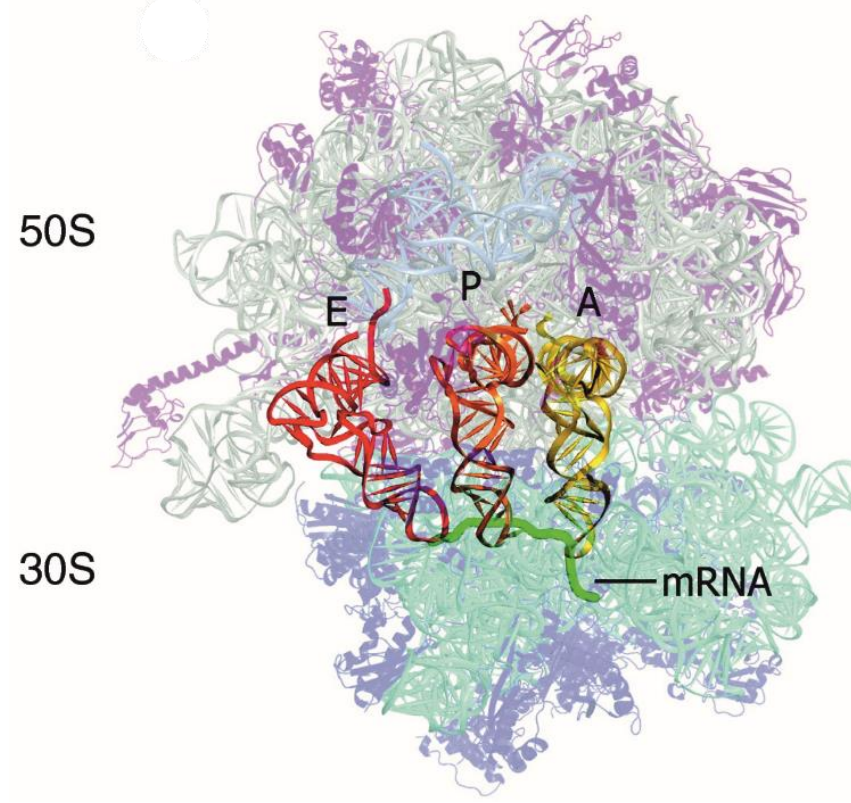

Figure 1 Ribosome structure

Figure adapted with permission from (Zhou et al., 2012). tRNA binding sites are denoted as E, P and A, the tRNA bound to these sites is in red, orange and yellow, respectively; rRNA is shown in shades of green, proteins in purple.

Additional protein translation factors associate with the ribosome at various stages of translation to assist in maintaining fidelity and processivity, while the tRNA is acting as a delivery vehicle for amino acids. Prokaryotic translation occurs in several stages called initiation, elongation, nascent peptide release (termination) and ribosome recycling (Rodnina, 2018). The eukaryotic translation has the same translation stages, however there are some differences. The Eukaryotic ribosomes are larger and contain additional rRNA and proteins (Ben-Shem et al., 2011). The initiation step involves more protein factors and is subject to multiple regulation mechanisms (Merrick and Pavitt, 2018). The eukaryotic mRNAs generally feature a modified guanine cap at the $5^{\prime}$ end, while the initiation is coordinated via specific initiation factors and the ribosome subunits recognizing and binding this modification (Merrick and Pavitt, 2018). The elongation cycle is the most conserved translation step across all kingdoms of life, however unique factors have evolved for specific requirements (Dever et al., 2018). Translation termination and peptide release generally occurs via similar mechanisms in prokaryotes and eukaryotes, but the eukaryotic termination is intertwined with mRNA quality control mechanisms and in many cases post-termination ribosomes can be interrupted at various recycling steps, leading to diverse conditions for rapid translation re-initiation (Hellen, 2018). The following chapters describe each of the prokaryotic translation steps. 


\section{Initiation}

The first step of translation is initiation, which is the selection of the correct starting position for peptide synthesis on a particular mRNA (Figure 2).
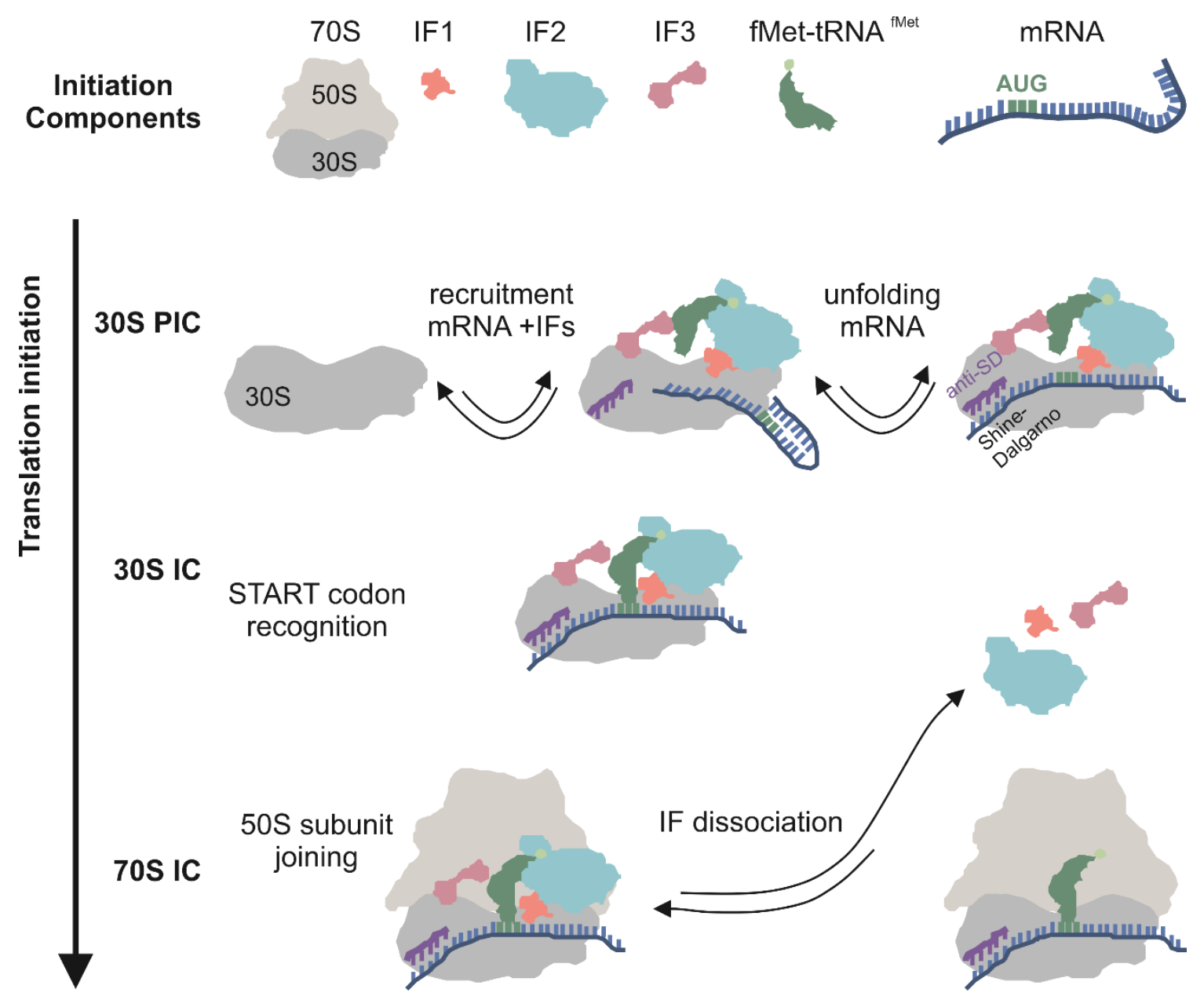

Figure 2 Translation Initiation

Scheme showing translation initiation in bacteria. 
Initiation factors (IF1, IF2 and IF3) associate with the 30 S subunit forming a pre-initiation complex (PIC), while also recruiting the initiator tRNA fMet-tRNA ${ }^{\mathrm{fMet}}$. The initiator tRNA is uniquely modified with a formyl group on the attached methionine. This serves to distinguish it from the Met-tRNA ${ }^{\text {Met }}$ participating in the elongation steps of translation (Milon and Rodnina, 2012). The joining of the mRNA to the PIC is not defined by the initiation factors and in many cases depends upon the interactions between small subunit rRNA and the mRNA itself (Figure 2). The anticodon of the fMet-tRNA ${ }^{\mathrm{fMet}}$ recognizes the starting codon AUG positioned in the decoding site of the $30 \mathrm{~S}$ ribosomal subunit. Most bacterial mRNAs have a Shine-Dalgarno (SD) sequence, which is located 6 to 10 nucleotides upstream of the translation start codon AUG. The SD sequence specifically interacts with the 16S rRNA of the 30S subunit, which helps to position the start codon in the $\mathrm{P}$ site. Establishing the codon-anticodon base pair interactions defines the open reading frame on the mRNA and indicates the formation of $30 \mathrm{~S}$ initiation complex (30S IC) (Milon and Rodnina, 2012; Simonetti et al., 2009) (Figure 2).

The docking of the 50S ribosomal subunit to the $30 \mathrm{~S} \mathrm{IC} \mathrm{depends} \mathrm{on} \mathrm{the} \mathrm{presence} \mathrm{of} \mathrm{initiation}$ factors and the initiator tRNA (Goyal et al., 2015). Once IF2 hydrolyzes GTP and the fMettRNA $^{\text {fMet }}$ goes through an accommodation step in the peptidyl-transferase center of the $50 \mathrm{~S}$ subunit, the initiation factors leave the complex. The subsequent formation of intersubunit bridges between the $30 \mathrm{~S}$ and $50 \mathrm{~S}$ ultimately traps mRNA and establishes the final $70 \mathrm{~S}$ initiation complex (Goyal et al., 2015; Goyal et al., 2017) (Figure 2). At each initiation step, the ribosome subunits and the associating factors undergo several conformational changes (Goyal et al., 2015; Rodnina, 2018) leading to the accurate positioning of the first tRNA on an mRNA, defining the open reading frame encoding a protein product and establishing the foundation for the translation elongation phase.

\section{Elongation}

During the elongation step sequential rounds of mRNA decoding, peptide bond formation and translocation produce a polypeptide (Figure 3). First, in the decoding step a codon exposed in the $A$ site of the ribosome is matched with the anticodon of an incoming amino aa-tRNA (Figure 3, Decoding). The aa-tRNA is delivered to the ribosome in a complex with elongation factor Tu (EF-Tu) which is a GTPase (Rodnina and Wintermeyer, 1995). The aa-tRNA-EF-Tu-GTP complex is called the ternary complex. At this stage the entire cellular pool of aa-tRNAs competes for the interaction with the A site codon. When a cognate ternary complex arrives to the ribosome, the match in codon-anticodon interactions between mRNA and tRNA triggers GTP hydrolysis in the EF-Tu-GTP-aa-tRNA complex (Rodnina, 2012; Rodnina and Wintermeyer, 2001a, b). The result of this is the dissociation of EF-Tu-GDP and the free 
phosphate from the aa-tRNA. Once free, the aa-tRNA undergoes an accommodation step, which positions the 3' CCA tail with the amino acid in the correct orientation at the $50 \mathrm{~S}$ subunit A site. The aa-tRNA is then primed for peptide bond formation (Rodnina et al., 2017). Elongation factor Ts (EF-Ts) is a nucleotide exchange factor for EF-Tu and will exchange the GDP to GTP, preparing EF-Tu for the binding of next aa-tRNA and the next round of decoding (Rodnina and Wintermeyer, 1995).

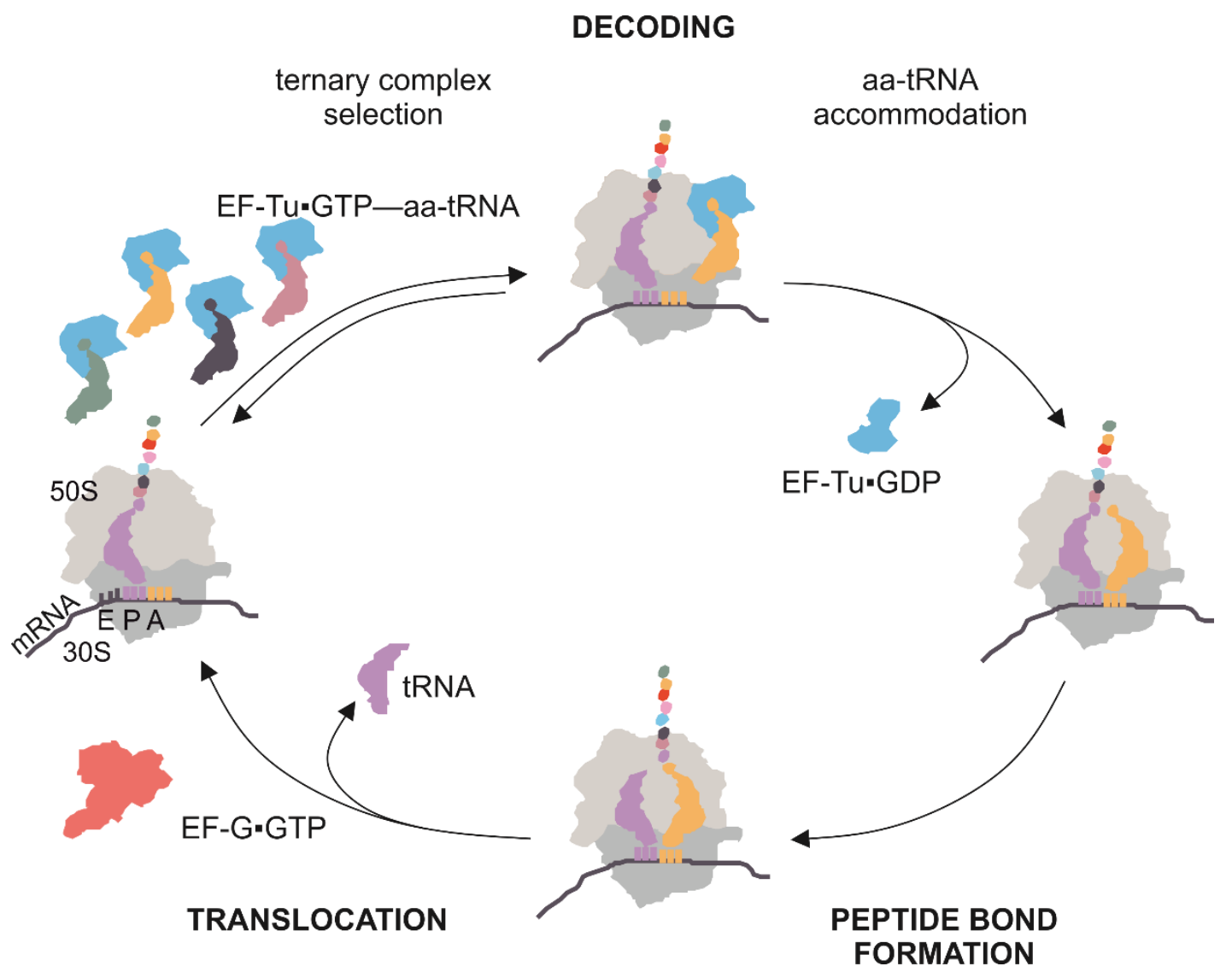

Figure 3 Elongation cycle

Scheme of the bacterial elongation cycle.

Second, during the peptide bond formation step a single amino acid is bound to a nascent peptide chain (Figure 3, peptide bond formation). The favorable conditions for peptide bond formation are created by the ribosome, where specific residues of the rRNA and the tRNAs are positioned in the correct reaction-favoring geometry. This allows a nucleophilic attack from the incoming $\alpha$-amino group of the A site aa-tRNA on the carbonyl group of the P site peptidyltRNA (Rodnina, 2013; Rodnina et al., 2006). Unlike protein enzymes, the ribosome contributes no catalytic groups, but rather provides electrostatic shielding, orders the water molecules in the PTC and orchestrates the intricate proton shuttling in the active site (Rodnina, 2018; 
Sharma et al., 2005; Wallin and Aqvist, 2010). The result is that the nascent peptide is now attached to the A-site tRNA and the P-site tRNA is deacylated.

The final step of the elongation cycle is translocation (Figure 3, Translocation). Translocation proceeds by disrupting interactions between the ribosome and the tRNA-mRNA thus allowing the forward movement of these elements through the ribosome (Rodnina, 2018). This step occurs through highly dynamic rearrangements of ribosomal subunits with respect to one another. The GTPase elongation factor G (EF-G) acts to provide directionality to the process by inserting itself into the $A$ site of the ribosome (Belardinelli et al., 2016). The deacylated $P$ site tRNA is positioned in the $E$ site and free to dissociate, the A site peptidyl-tRNA enters the $P$ site, and the A site is empty with a new codon present in the decoding center (Figure 3 ). The ribosome is ready for a subsequent round of elongation.

The rate of elongation for a particular mRNA can vary with each codon, because the kinetics of decoding depend on the binding kinetics of each tRNA and in the cellular tRNA concentrations are not uniform. Specific features of translation kinetics can be utilized in translational recoding events such as frameshifting (Korniy et al., 2019) and are important in maintaining the quality of synthesis products - stable natively folded proteins (Buhr et al., 2016).

\section{Peptide Release and Recycling}

Translation enters its final stage when the ribosome reaches a stop codon on the mRNA. Three codons signal translation termination in bacteria - UAG, UAA, and UGA. There are two release factors: RF1 and RF2, both of which recognize the UAA stop codon; the UAG is exclusively recognized by RF1 and UGA by RF2 (Klaholz, 2011) (Figure 4). An associated release factor RF3 participates in the RF1 and RF2 turnover after translation termination (Adio et al., 2018; Peske et al., 2014). RF1/RF2 recognize their respective stop codons via conserved residues forming protein-RNA interactions (Noller et al., 2011). Both of these proteins extend into the 50S PTC positioning a universally conserved functional GGQ motif that helps the ribosome carry out the hydrolysis of the ester bond between the tRNA and the nascent peptide chain (Figure 4). The peptide is released from the ribosome and the ribosome itself is recycled for the subsequent round of translation. 


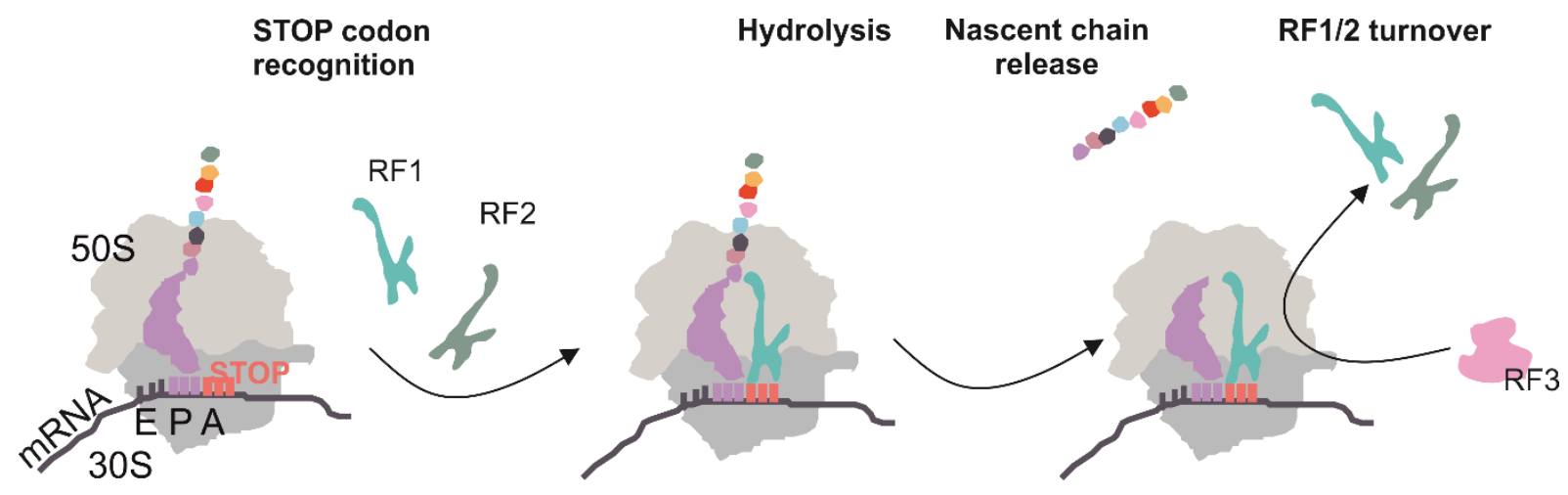

Ribosome recycling

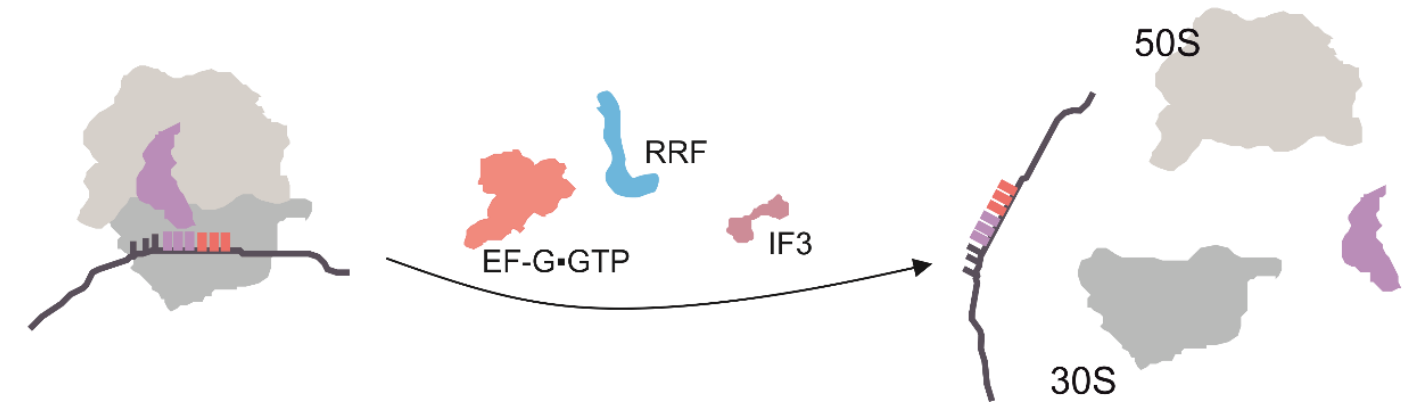

Figure 4 Release and Recycling

A scheme depicting translation termination and ribosome recycling.

During the recycling step, the subunits have to be split and the mRNA and tRNA released from the ribosome to allow a new round of initiation (Figure 4). The subunit splitting is carried out by ribosome recycling factor (RRF) and EF-G working together (Gao et al., 2005; Peske et al., 2005). The $30 \mathrm{~S}$ subunit is then left with the tRNA and mRNA. Initiation factor IF3 displaces the tRNA from the 30S subunit, while the mRNA exchange occurs spontaneously (Fu et al., 2016; Peske et al., 2005; Rodnina, 2018).

The purpose of translation is to express the genetic information encoded in the cellular DNA to active and intact cellular operatives - proteins. The capacity of resulting proteins to execute their functions depends not only on the fidelity of translation into the linear polypeptide, but also on the final three-dimensional structure that the protein can reach in the cellular environment. How the final three-dimensional structure is achieved and the type of phenomena that drive protein folding will be discussed in the following chapters. 


\section{Protein folding}

The protein structure hierarchy encompasses four levels. Primary structure is the linear amino acid polymer (Figure 5). This can be organized into secondary structure elements such as ahelixes and $\beta$-sheets stabilized by hydrogen bonding between peptide backbone atoms. Different amino acid residues have different propensities for specific secondary structures (Griffiths-Jones et al., 1998; Swindells et al., 1995). Interactions between individual secondary structure elements form tertiary structures (Figure 5) which are stabilized by interactions between the amino acid side chains based on electrostatic and hydrophobic properties, van der Waals interactions between atoms, and disulfide bridges formed by cysteine residues. Interactions between tertiary structures are called quaternary structures that emerge when several proteins oligomerize to form a functional unit (Figure 5).

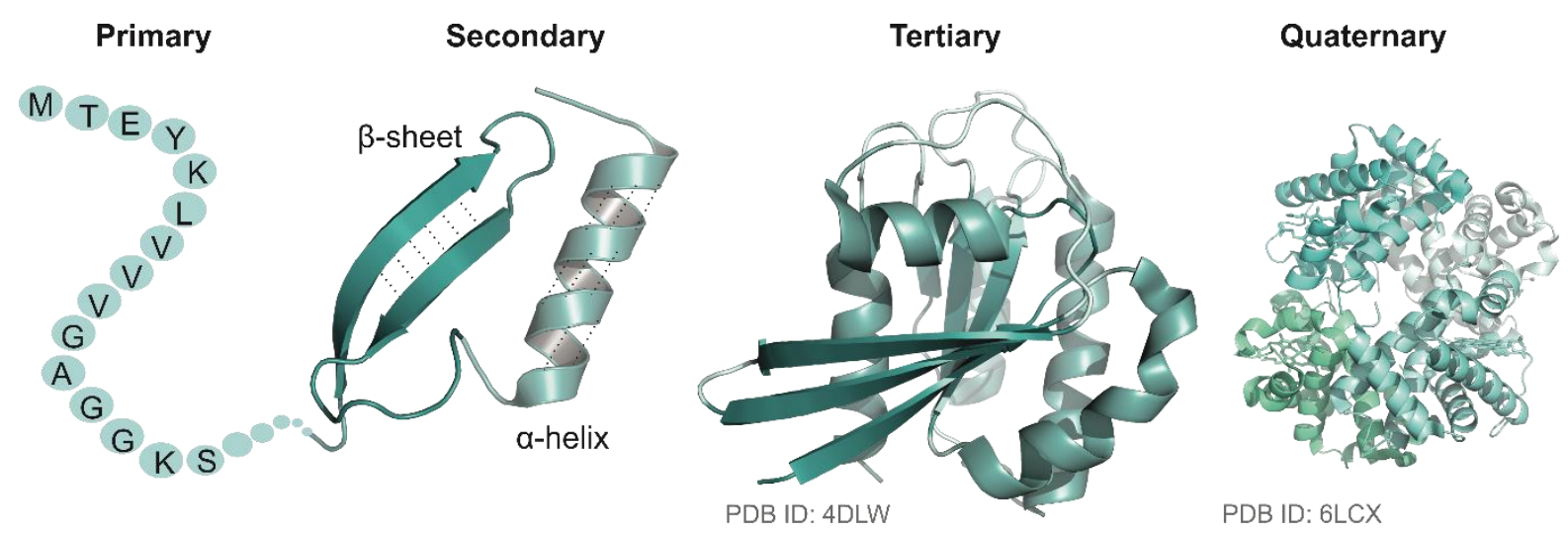

Figure 5 Hierarchy of protein structure

Dashed lines indicate hydrogen bonding between atoms of the polypeptide backbone. Quaternary structure - each shade is a separate polypeptide chain.

If a simple 101 amino acid-long protein had to sample at least three conformations per residue, taking a $10^{-13} \mathrm{~s}$ at each conformation, then it would take $10^{27}$ years for that protein to reach its native conformation (Levinthal, 1969). In this time, a photon could travel the diameter of the known universe $10^{17}$ times (Bars and Terning, 2010). This seemingly infinite number of conformations accessible to a polypeptide is known as Levinthal's paradox. However, experiments with protein folding show that majority of proteins will complete their folding in seconds to minutes (Anfinsen et al., 1961; Garbuzynskiy et al., 2013). This means that there are additional phenomena that drive protein folding instead of a blind infinite search for the correct configuration on the level of individual amino acids.

There are several experimentally verified nearly universal features of protein folding. First, for most proteins folding and unfolding is a reversible process (Anfinsen, 1973; Shakhnovich and 
Gutin, 1990) and both of these can be observed at conditions when the unfolded (U) and folded native $(\mathrm{N})$ states exist at an equilibrium and have equal stabilities (Fersht et al., 1999; Shakhnovich and Gutin, 1990). In cases of complex folding trajectories, during refolding a protein may be sequestered in partially folded stable states for extended periods, under experimental conditions this can lead to aggregation, however given enough time folding to native state remains possible (Finkelstein and Ptitsyn, 2016d). Second, the $U$ state protein can exist in a variety of thermodynamic states with various properties but the transitions between these states are minor compared to any $U$ state transition to the final $N$ state (Ivankov and Finkelstein, 2020). Many complex large proteins and even some small and relatively simple proteins fold via intermediate states (I). The most characterized and studied intermediate state is the molten globule. It is defined by a loosely packed protein core that contains water molecules and has more conformational freedom when compared to the $\mathrm{N}$ state (Finkelstein and Ptitsyn, 2016c). Finally, the transition between the $\mathrm{N}$ and $\mathrm{U} / \mathrm{I}$ states happens in all-ornothing mechanism - from inactive and unfolded to a native biologically functional protein (Privalov, 1979). This means that the energy difference between the ensemble U/I states and the $\mathrm{N}$ state has to be a sizeable barrier to ensure that the $\mathrm{N}$ state will be the most energetically favorable (Galzitskaya and Finkelstein, 1995; Sali et al., 1994; Shakhnovich, 2006; Shakhnovich and Gutin, 1990). Nonetheless, in the cell proteins exist in a generally shallow energy landscape, meaning that the lowest energy native state is more stable than the unfolded state or the nearest intermediate only by tens of kilojoules (Gruebele et al., 2016; Taverna and Goldstein, 2002).

Several conceptual models exist (Figure 6) that describe how proteins can fold with high speeds, accuracy and why a time demanding exploration of each amino acid conformation is not necessary to reach the final structure.

\section{Folding Model: Framework (diffusion collision)}

The framework folding model suggests a more hierarchical folding trajectory (Baldwin and Rose, 1999a, b). First, the fastest folding elements, i.e., the secondary structures $\alpha$-helixes and $\beta$-sheets, would form. These would then be able to find the tertiary interaction partners through randomly diffusing and colliding to form interfaces (Figure 6, Framework). The final step is the expulsion of all water from the hydrophobic core to allow for the tight side-chain packing in native structures of globular proteins (Bashford et al., 1988; Karplus and Weaver, 1976, 1994; Kim and Baldwin, 1982; Udgaonkar and Baldwin, 1988). This model of folding demands that a protein crosses several energy barriers. Additionally, there seems to be no 
necessity for a set sequence of secondary structure docking, suggesting a multitude of pathways and various energy barriers for a protein to reach the native state (Karplus and Weaver, 1994).

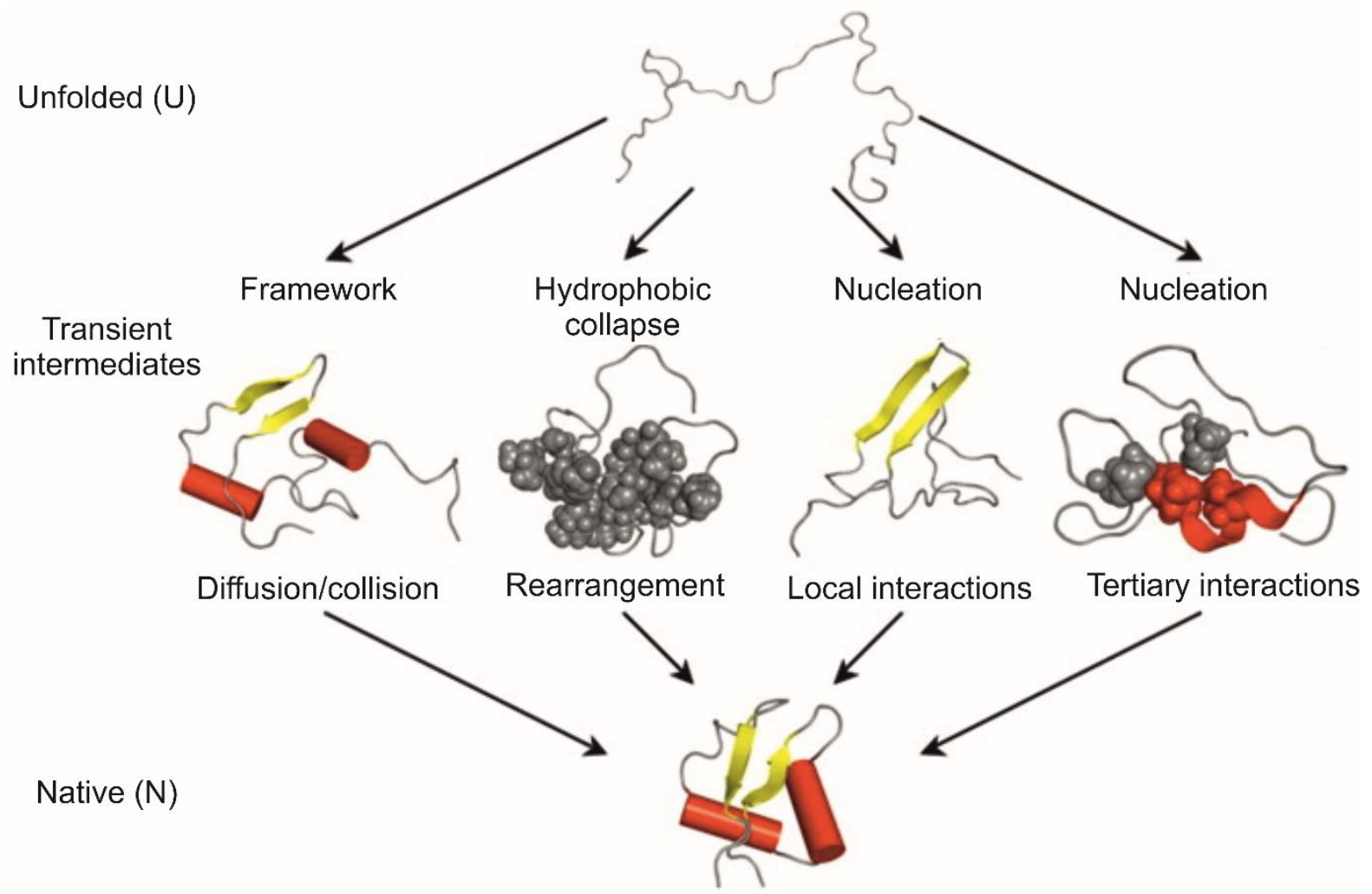

Figure 6 Protein folding models

Adapted with permission from (Nickson and Clarke, 2010).

\section{Hydrophobic Collapse}

This model relies on early forming hydrophobic interactions among hydrophobic amino acids (Figure 6, Hydrophobic collapse). Folding begins with an initial clustering of hydrophobic residues that energetically favor a non-aqueous environment (Dolgikh et al., 1981). This initial step appears to have none or a very small energy barrier for formation (Gruebele, 2005; Sinha and Udgaonkar, 2007). The relative hydrophobicity of different amino acids is different (Figure 7) (Finkelstein and Ptitsyn, 2016b) and so the strength of initial interaction would vary depending on the particular residues in the cluster. Once the initial and non-specific hydrophobic amino acid cluster is established, the conformational space for the remaining polypeptide is significantly reduced (Gutin et al., 1995; Robson and Pain, 1971; Sadqi et al., 2003). This would lead to the formation of secondary structures that could then form tertiary interactions through local motions in this restricted conformational space. Ultimately, the 
protein can undergo a final rearrangement that results in the native state (Agashe et al., 1995; Dill et al., 1995; Sinha and Udgaonkar, 2007).

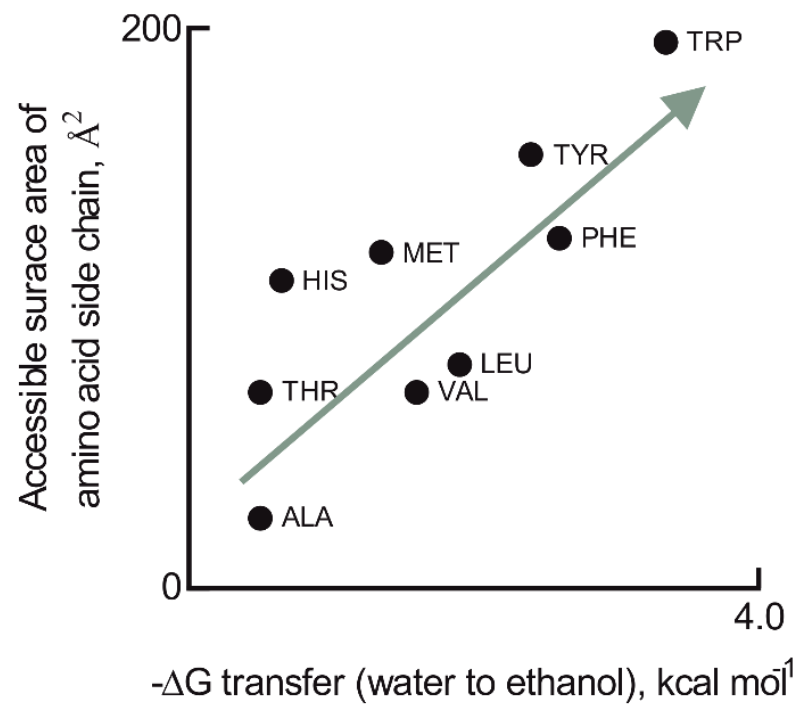

\section{Figure 7 Amino acid hydrophobicity}

Amino acid hydrophobicity shown with the change in free energy plotted as a function of accessible surface area (Finkelstein and Ptitsyn, 2016b). The larger the amino acid surface area the higher the energetic cost of this amino acid being in an aqueous environment - green arrow indicates increasing hydrophobicity.

\section{Folding Model: Nucleation}

The nucleation model suggests that the folding initiates as a few key residues form a secondary structure nucleus (Figure 6, Nucleation, local interactions). This initial nucleation is the ratelimiting step of folding and once it is established, the native structure grows around it (Wetlaufer, 1973). A variation of this model is the nucleation-condensation model (Figure 6, Nucleation, tertiary interactions). In the cases when individual secondary structures alone are unstable and cannot remain folded while the rest of the protein "grows" around it, tertiary interactions contribute to the stability of the folding nucleus. In this case, the folding nucleus is also more diffuse, meaning that the tertiary interactions and secondary structure formation are happening in a concerted manner (Daggett and Fersht, 2003; Fersht, 1995, 1997; Itzhaki et al., 1995). In both of these cases, a high energy transition state has to be traversed to establish a folding nucleus, which is in stark contrast to the energetically barrier-less formation of the first folding unit in the hydrophobic collapse model (Jha and Udgaonkar, 2010). 


\section{Energy Landscape Theory}

Energy landscapes are visualizations of a protein entropy (disorder) and its enthalpy (free energy) (Figure 8). It shows how the conformational space for a given protein changes, during the folding process. At the highest free energy state (the opening of the funnel), the entropy is very high, but as soon as secondary and tertiary structures start to form, the free energy decreases and the conformational space for a particular protein also decreases (Figure 8). However, the overall system disorder will grow, because fewer water molecules will be engaged in hydrogen bonding with the polypeptide (Finkelstein and Ptitsyn, 2016a). The movement down the folding funnel is the trajectory of protein folding, but for each protein, this landscape will be different due to different numbers and combination of amino acids in the polypeptide and different entropic properties. The energy landscape, however, does not account for the chemical properties of amino acids like hydrophobicity or charges, rather encompassing these effects under the "entropy" of the entire polypeptide. The energy landscape theory is often used to explain experimental observations, but cannot predict folding trajectories or rates (Jha and Udgaonkar, 2010). It also generally views folding as a downhill process, devoid of significant free energy barriers (Gruebele, 2005). It is able to visualize and distinguish two-state folding models, where no intermediate states can be detected, and threestate folding models, where a distinguishable intermediate state is populated and can be detected (Morris and Searle, 2012) (Figure 8).

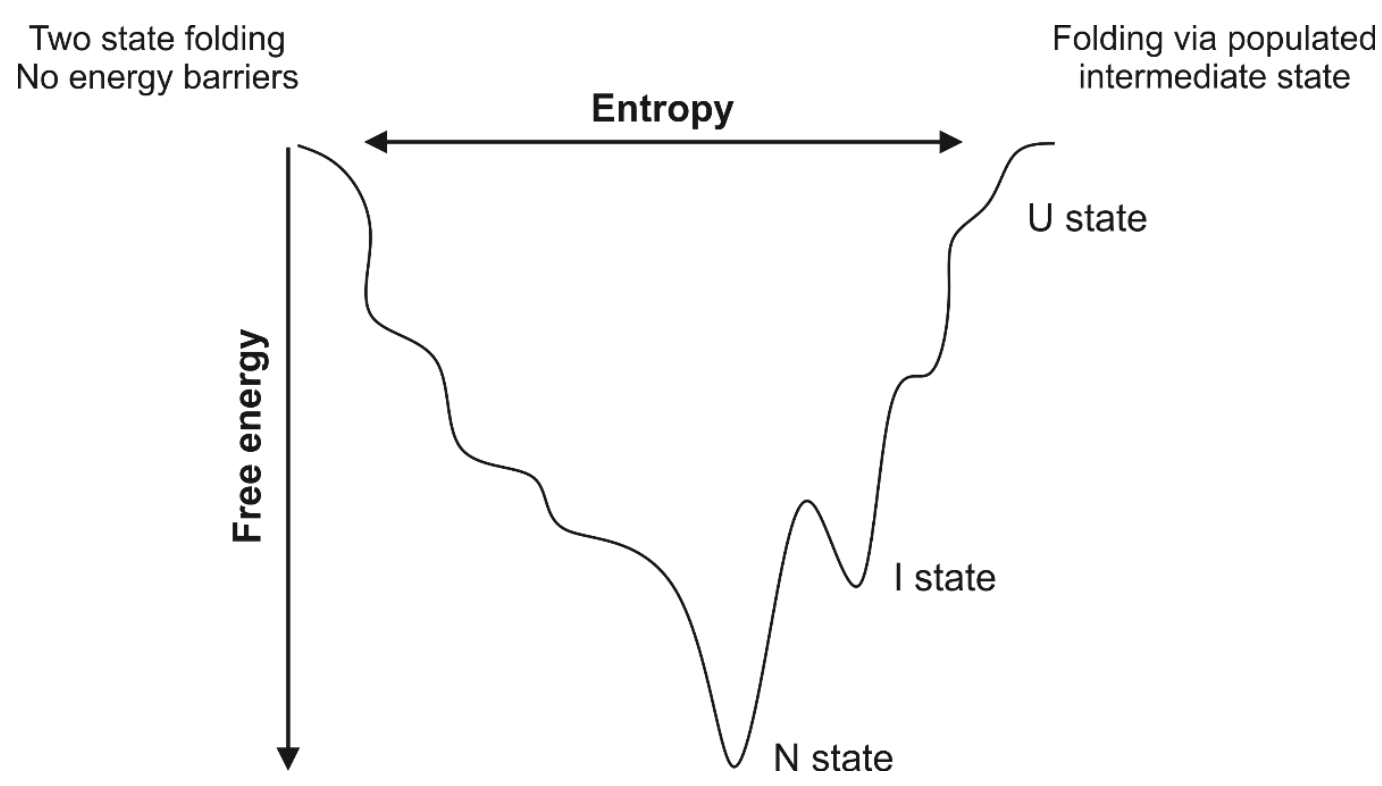

\section{Figure 8 Folding energy landscape}

A two-dimensional representation of a folding funnel energy landscape. On the left, protein folding in a two-state mechanism $U$ to $\mathrm{N}$ without significant energy barriers is visualized. On the right, folding is via a three-state mechanism $U$ to I to $N$, where I is a populated on-pathway intermediate. The diagram shows the changes in the free energy and entropy as a protein folds traversing the energy landscape. 
In all of the discussed protein folding models, the polypeptide chain undergoes several structural rearrangements. These structural rearrangements involve breakage and formation of various intra- and inter-molecular interactions e.g. hydrogen bonding between amino acids and with water molecules, additionally hydrophobic packing of side chains, as well as electrostatic interactions between side chains and the van der Waals contacts might have to be disrupted and reestablished. Folding is driven by the thermodynamic requirement that the entire protein and solvent system must reach a minimum energy and maximum entropy state - any folding barriers will arise from the incomplete compensation of one or the other (Jha and Udgaonkar, 2010).

Protein's failure to fold, leaving large exposed hydrophobic patches, leads to aggregation. The shallow energy landscape in which proteins exist (Taverna and Goldstein, 2002) means that changes in temperature, $\mathrm{pH}$, or amino acid incorporation mistakes during translation can result in changes of folding equilibrium, creating unfolded or aberrantly folded proteins (Gregersen et al., 2006). These proteins can form oligomers, amyloids or amorphous aggregates taxing the cellular machinery, triggering apoptotic cascades, causing disruptions in cellular functions and resulting in diseases (Powers et al., 2009; Santra et al., 2019). Understanding the fundamental aspects of protein folding helps to understand the key cellular events like aging, death, and pathologies of diseases.

\section{Methods used to study protein folding}

Many biophysical methods have been developed to probe and understand the thermodynamic and kinetic aspects of protein folding trajectories and how the folding barriers are traversed by different proteins. The most detailed structural information on the native state of a protein can be obtained using structural methods like X-ray crystallography, nuclear magnetic resonance (NMR) and more recently cryogenic electron microscopy (cryo-EM) ((Dobson, 2019) and references therein). Both cryo-EM and X-ray crystallography can provide atomic resolutions of static protein structures, whereas NMR is uniquely used to visualize macromolecular structures in their native environments and investigate the dynamic fluctuations of proteins (Dyson and Wright, 2004; Marion, 2013). These methods are mainly used to study the highly populated and stable native states of proteins.

Spectroscopic techniques can be applied to investigate the transitions of proteins from unfolded to folded states. Typically, chemical, temperature, or pressure denaturation experiments are performed monitoring the protein in its native, fully denatured and 
intermediate states at equilibrium to determine the differences in free energies and transition state barriers (Morris and Searle, 2012). Rapid denaturing perturbations followed by timeresolved monitoring of the recovery of protein to its native state can be used to determine the kinetic folding parameters (Morris and Searle, 2012).

Monitoring the intrinsic tryptophan fluorescence can report on the local tertiary structure around the residue (Royer, 2006). Far-UV and near-UV circular dichroism spectroscopy is often used to study the total secondary structure content and to what degree the tertiary structures form around the aromatic residues (phenylalanine, tyrosine ant tryptophan) (Greenfield, 2006a, b, c). Measuring the UV absorbance of aromatic residues in the polypeptide also allows investigating the local tertiary structure formations around a particular residue (Lange and Balny, 2002). Time-resolved and steady-state fluorescence anisotropy measurements can be used to understand the degree of immobilization or freedom for particular tryptophan residues in the polypeptide (Canet et al., 2001). This freedom changes at different states of protein folding: in largely unfolded structures, the tryptophan would have high anisotropy values, while in compact native states the tryptophan will have low anisotropy values (Beechem et al., 1995; Canet et al., 2001; Fa et al., 1995). The specific amount of rotational freedom and the changes in that freedom depends on the location of the tryptophan residue (Sridevi et al., 2000). Finally, infrared spectroscopy is used to investigate the amide groups in the backbone of the protein, thus mostly reporting on the secondary structure changed during global unfolding (Fabian and Naumann, 2004; Kong and Yu, 2007).

Mutagenesis studies in combination with the outlined techniques can identify which particular amino acids contribute to the transition state structure during protein folding. The kinetic and thermodynamic parameters of the wt protein are compared to a protein with a point mutation. This comparison yields phi values, which indicate the energetic contribution of that particular residue to the folding transition state (Matouschek et al., 1989).

It is now possible to map extremely short-lived intermediate structures that arise during protein folding. Hydrogen exchange pulse labeling targets the amides in the protein backbone, exchanging the hydrogen with a deuterium under various conditions (Krishna et al., 2004). Under specific destabilizing solvent conditions or at a specific folding time point different parts of the protein backbone are exposed and deuterium-labelled. To analyze which parts of the protein gain protection from the exchange at different points along the folding pathway NMR and/or mass-spectrometry techniques can be used (Englander and Mayne, 2014). This method provides information on protein folding trajectory allowing the identification of various 
short-lived intermediates at nearly amino acid resolution (Englander and Mayne, 2014; Hu et al., 2013; Walters et al., 2013).

Force spectroscopic methods, such as atomic force microscopy and optical tweezers, are utilized to study unfolding of single protein molecules at a time (Neuman and Nagy, 2008). In these experiments, the usual chemical, $\mathrm{pH}$ or temperature denaturants are replaced with mechanical forces and the unfolding forces are applied to the termini ( $\mathrm{N}$ or $\mathrm{C}$ terminus) of a protein (Lenne et al., 2000; Rief et al., 1997; Yang et al., 2000) . These experiments provide information on how different folds of proteins respond to mechanical tensions; how much force is necessary to unfold entire proteins or protein subdomains; how robust are unfolding and refolding pathways for the same protein (Best and Clarke, 2002).

To understand the heterogeneity of protein folding intermediates further, single-molecule fluorescence spectroscopy techniques can also be used. Fluorescent probes are extremely sensitive to the chemical environment and provide information that is detectable at a single molecule level (Eftink, 1994; Michalet et al., 2006). During different stage of folding the environment of the protein changes, e.g., hydrophobic residues are buried in the protein core, charged residues form electrostatic interactions, cysteines form disulfide bridges, etc., thereby creating changes in the fluorescence signals. Specific fluorophores can provide time information on the difficult to capture sparsely populated transition states (Chung et al., 2012), as well as information ranging from the very fast $\mu$ s dynamics at the unfolded intermediate states to slow oligomerization of multiple folded proteins that takes hours (Schuler and Hofmann, 2013). Fluorescence resonance energy transfer (FRET) (Deniz et al., 2000; Zhuang et al., 2000) and photo-induced electron transfer (PET) reporters (Doose et al., 2009; Noe et al., 2011) can cover a range of distances on the nascent protein from hundreds to single Ångstroms (Schuler and Hofmann, 2013).

Our understanding of the fundamental processes of protein folding has been forged from biophysical experiments on isolated proteins in vitro or in silico computer simulations (reviewed in detail (Gershenson et al., 2020; Huang et al., 2016; Kuhlman and Bradley, 2019; Scheraga et al., 2007; Snow et al., 2005)). The main reason for this is that in vivo folding begins during protein synthesis and the analysis of this process would require techniques that could specifically target the emerging nascent polypeptide chain and deconvolute the signal from the RNA and proteins that comprise the translation machinery. Only a subset of methods can achieve this (Komar, 2018), such as single molecule fluorescence methods with specifically labelled nascent proteins, force spectroscopy methods, and structural methods like cryo-EM and NMR. All of these require robust sample preparation of the nascent chains still associated with the ribosome, and in some cases the quantity of sample required can pose significant 
challenges (NMR) (Komar, 2018). The recent work within the protein folding field during translation is discussed in the following section.

A particularly promising tool to study the dynamic motions of cotranslationally arising intermediates is the fluorescence correlation spectroscopy (FCS) coupled with photoinduced electron transfer reporters (PET-FCS) (Figure 9). The resolution of a confocal FCS setup ranges from nanoseconds to milliseconds, and fluorescence fluctuations are monitored as a fluorophore or a fluorophore-labelled protein diffuses through the illuminated volume (Figure 9) (Krichevsky and Bonnet, 2002).
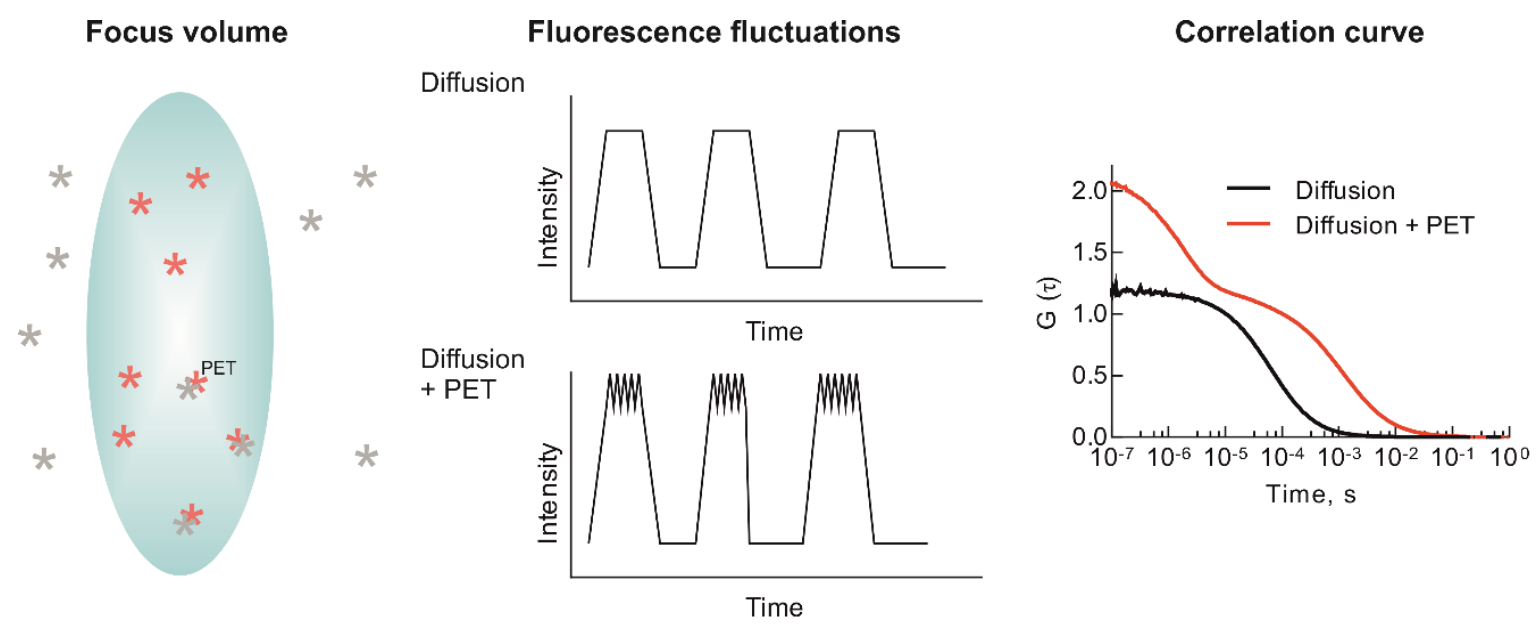

\section{Figure 9 Fluorescence correlation spectroscopy}

A schematic of FCS workflow. The labelled molecules diffuse through the focus volume of a confocal microscope; fluorescence intensity fluctuations are recorded and transformed to autocorrelation curves for analysis (Sauer and Neuweiler, 2014).

A PET reporter (Doose et al., 2009) can be utilized to monitor short-range structural fluctuations that occur during the molecule's residence in the illuminated volume (Neuweiler et al., 2003b; Sauer and Neuweiler, 2014) . When fast structural fluctuations bring a fluorophore and a native tryptophan residue into van der Waals contacts, fluorescence quenching occurs. In contrast, a fluorescence signal appears if these residues are separated (Doose et al., 2009) (Figure 9). These fast fluorescence fluctuations report on structural fluctuations of a particular protein and once calculated using an autocorrelation function can provide information on the timescales of these motions (Neuweiler et al., 2003b; Sauer and Neuweiler, 2014). PET-FCS has been used to study the dynamic qualities of proteins in solution (Doose et al., 2007; Luitz et al., 2017; Lum et al., 2012b; Neuweiler et al., 2010a; Neuweiler et al., 2009). In principle, it 
should be feasible to apply this technique to study the dynamics of nascent proteins associated with the ribosome. This would require the labelling of nascent chain with a fluorophore and the presence of a tryptophan quencher the accessibility of which would change during different stages of protein folding. 
SECTION 2 - Cotranslational Folding 


\title{
Cotranslational Folding of Proteins on the Ribosome
}

\author{
Marija Liutkute, Ekaterina Samatova * and Marina V. Rodnina *(1) \\ Department of Physical Biochemistry, Max Planck Institute for Biophysical Chemistry, \\ 37077 Göttingen, Germany; mliutku@mpibpc.mpg.de \\ * Correspondence: esamato@mpibpc.mpg.de (E.S.); rodnina@mpibpc.mpg.de (M.V.R.); \\ Tel.: +49-551-201-2900 (E.S. \& M.V.R.); Fax: +49-551-201-2905 (E.S. \& M.V.R.)
}

Received: 5 December 2019; Accepted: 25 December 2019; Published: 7 January 2020

\begin{abstract}
Many proteins in the cell fold cotranslationally within the restricted space of the polypeptide exit tunnel or at the surface of the ribosome. A growing body of evidence suggests that the ribosome can alter the folding trajectory in many different ways. In this review, we summarize the recent examples of how translation affects folding of single-domain, multiple-domain and oligomeric proteins. The vectorial nature of translation, the spatial constraints of the exit tunnel, and the electrostatic properties of the ribosome-nascent peptide complex define the onset of early folding events. The ribosome can facilitate protein compaction, induce the formation of intermediates that are not observed in solution, or delay the onset of folding. Examples of single-domain proteins suggest that early compaction events can define the folding pathway for some types of domain structures. Folding of multi-domain proteins proceeds in a domain-wise fashion, with each domain having its role in stabilizing or destabilizing neighboring domains. Finally, the assembly of protein complexes can also begin cotranslationally. In all these cases, the ribosome helps the nascent protein to attain a native fold and avoid the kinetic traps of misfolding.
\end{abstract}

Keywords: cotranslational protein folding; ribosome; polypeptide exit tunnel; nascent polypeptides; translation; protein synthesis

\section{Introduction}

Proteins are a key class of biological macromolecules that are essential in all cellular processes. To execute their functions and maintain the cell viability, proteins have to fold into their specific native three-dimensional structures. Misfolding disturbs the cellular proteostasis, which can result in debilitating diseases [1-3]. Single amino-acid substitutions can disrupt a protein's structure in the cell to cause, for instance, cystic fibrosis [4], sickle cell anemia [5], cataract [6], Huntington's disease [7], or retinitis pigmentosa [8]. The molecular pathology of these diseases is a perturbation of the native three-dimensional structure leading to a misfolded protein that can no longer execute its function and is prone to aggregation and rapid degradation. Furthermore, mutations in natively disordered proteins, such as $\alpha$-synuclein, tau protein or amyloid $\beta$-peptide, can cause aggregopathies, such as Parkinson's and Alzheimer's [2].

Many proteins start to fold cotranslationally as they move through the peptide exit tunnel and emerge from the ribosome (Figure 1). About one third of the E. coli proteome is estimated to fold cotranslationally [9]. The average rate of protein synthesis is $\sim 20$ amino acids/s in E. coli [10] and $\sim 6$ amino acids/s in eukaryotic cells [11,12]. In comparison, experimentally measured rates of spontaneous folding of single-domain globular proteins range from microseconds to hours [13]. In cases where translation is slower than folding, cotranslational protein folding takes place at quasi-equilibrium conditions [14]. The ribosome can destabilize nascent folds and delay folding until the entire domain is exposed $[15,16]$. The vectorial nature of protein synthesis, as well as the restricted space and the 
physicochemical properties of the exit tunnel [17] can determine the onset of folding and define the folding landscape, thereby guiding the folding trajectory away from kinetic traps and towards stable productive conformations. The N-terminus of the emerging nascent peptide can interact with ribosome-bound chaperones, protein biogenesis factors, cofactors or partners in multi-subunit complexes, thereby ensuring correct protein localization, activity and preventing erroneous associations with proteins in the crowded cellular environment [18] (Figure 1).

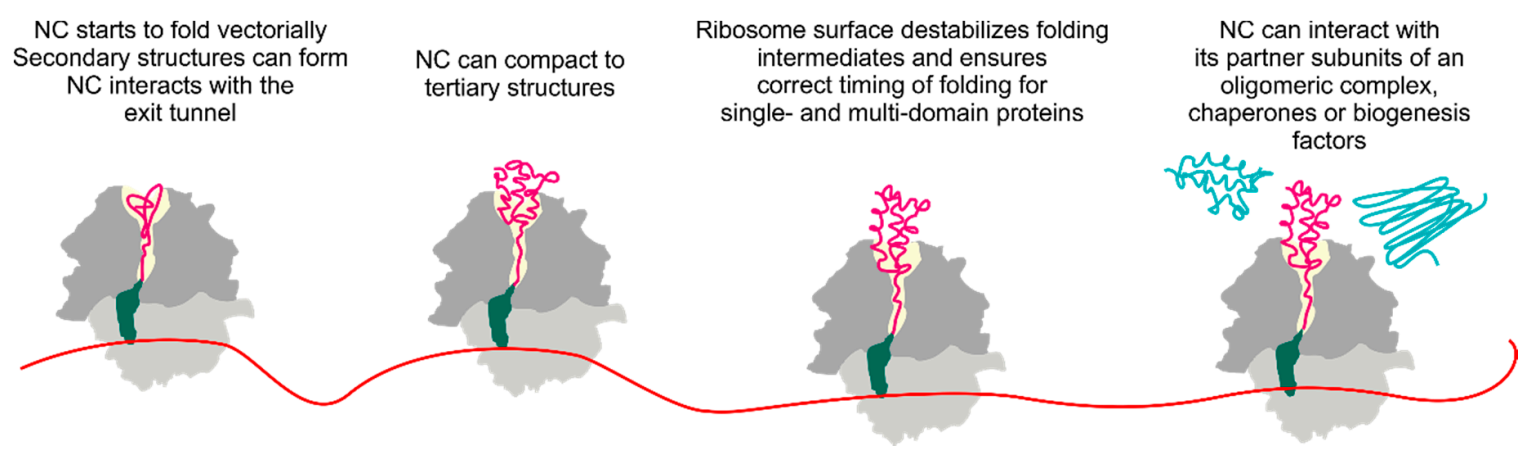

Figure 1. Schematic of cotranslational protein folding. Folding begins early inside the polypeptide exit tunnel. The nascent chain (NC) emerging from the ribosome can interact with chaperones, biogenesis factors, or other proteins. Small and large ribosomal subunits are shown in light and dark gray, respectively; the tRNA (green) with the nascent peptide (magenta) is shown as the ribosome moves along the mRNA (red) and the growing nascent chain moves through the polypeptide exit tunnel (light yellow). Protein partners interacting with the nascent peptide are depicted in blue.

Early in vitro protein refolding experiments have shown that the amino acid sequence carries all information required for small globular proteins to fold into their correct native states [19]. However, cotranslational protein folding can begin when only an N-terminal segment of the protein is available, before the C-terminal part is synthesized [20] (Figure 1). This raises the question whether the folding pathway is the same on and off the ribosome. Furthermore, large multi-domain proteins often fail to refold correctly in solution, resulting in misfolded structures and aggregation. For such proteins, domain-wise cotranslational folding may reduce the probability for off-pathway and aggregation-prone conformations [21,22], accelerate folding into the native state or even alleviate the need for chaperone assistance [20,23-25]. Many proteins are a part of multi-subunit complexes. These proteins not only have to adopt their individual native structures, but also to find their interaction partners in the crowded cellular environment. Cotranslational folding also plays an important role in coordinating the biogenesis of oligomeric proteins [26] (Figure 1), underscoring the importance of cotranslational events for biogenesis of different types of protein structures. A peptide emerging from the exit tunnel is monitored by ribosome-associated chaperones and protein biogenesis factors, which control folding and ensure the correct processing and cellular localization of proteins.

In this review, we summarize current concepts of cotranslational protein folding, focusing on how the ribosome affects folding and how single-domain, multiple-domain, and oligomeric proteins fold. Other aspects of co- and post-translational folding, such as the role of chaperones and protein biogenesis factors, folding of membrane proteins, as well as the link between the rate of translation and folding, are covered by recent comprehensive reviews [27-30].

\section{The Environment of the Peptide Exit Tunnel}

The peptide exit tunnel of the ribosome provides a confined space where the nascent chain begins to fold. The tunnel starts at the peptidyl transferase center (PTC) and extends for $\sim 100 \AA$ through the large ribosomal subunit before opening into the cytosol [31-34] (Figure 2). The tunnel is composed mainly of the ribosomal RNA (23S rRNA in bacteria and 28S rRNA in eukaryotes). Two ribosomal proteins, uL4 and uL22, of the large ribosomal subunit form a constriction of the tunnel $\sim 30 \AA$ away 
from the PTC, which is found in ribosomes from all domains of life. In addition, eukaryotic ribosomes have a second constriction formed by the extended arm of $\mathrm{uL} 4$ protein in the lower part of the exit tunnel [34] (Figure 2). The tunnel width varies between 10 and $20 \AA$ and becomes wider $\sim 50 \AA$ away from the PTC. The last $20 \AA$ of the tunnel form the so-called vestibule, which is generally wider than the rest of the tunnel and is shaped by proteins uL23 and uL24 in bacteria and additionally eL39 in eukaryotes (Figure 2). Residues lining the exit tunnel are highly conserved in the zone proximal to the PTC, whereas those in the vestibule have the most variation, with the tunnel in bacteria overall being wider than in eukaryotes [34]. The tunnel shields about 30-40 amino acids of the nascent peptide in the upper $80 \AA$ of the tunnel from proteolytic digestion [35,36], although the length of the protected nascent chain may depend on the extent of cotranslational folding inside the tunnel [37].

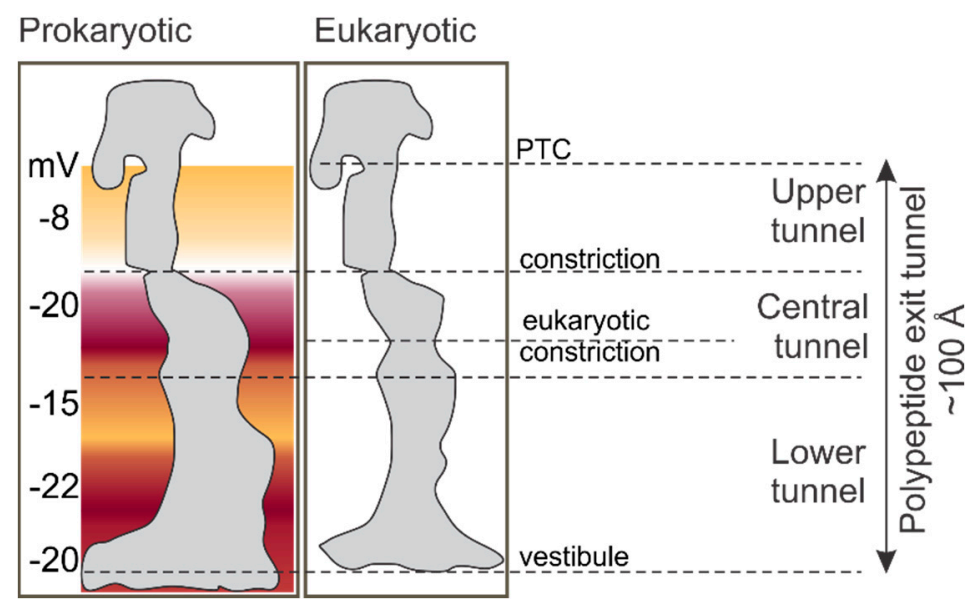

Figure 2. Dimensions of the peptide exit tunnel in ribosomes from prokaryotic and eukaryotic origin $[34,38]$. Color visualizes the electrostatic potential within the tunnel.

Molecular dynamics simulations suggest that inside the exit tunnel the water is in a slowly-diffusing and semi-structured state different from the bulk or tightly bound water [39]. The water properties inside the exit tunnel may slow down diffusion and favor specific conformations of the nascent chain [39]. For hydrophobic nascent chains, the layer of water molecules between the nascent chain and the hydrophilic tunnel walls may drive nascent-chain compaction. By contrast, a polar nascent chain in this same situation would experience a smaller drive to form helical structures, but would rather displace the ordered solvent molecules on the surface of the tunnel resulting in close contact between the nascent chain and the tunnel walls [39]. Biochemical and structural studies suggest that nascent chains may form helical structures in the upper regions of the tunnel, even though a peptide with the same amino acid sequence in solution does not form a stable helix $[37,40,41]$.

Aside from restricting the folding space, the peptide exit tunnel provides a characteristic electrostatic environment. Ribosomal proteins and rRNA that line the tunnel walls contribute to the global electrostatic potential of the tunnel. On average, the tunnel is more negatively charged than the cellular matrix [38]. The charge is unevenly distributed and varies from $-8 \mathrm{mV}$ to $-22 \mathrm{mV}$ along the length of the tunnel [38] (Figure 2). The lowest potential, $-20 \mathrm{mV}$, is found at the constriction near the uL4 and uL22 proteins. The high degree of conservation of the rRNA sequence and of the charged amino acids lining the tunnel walls suggests that the electrostatic properties of the tunnel are functionally important [34]. In fact, experiments with ribosomal protein S6 as a model nascent chain suggest that changing the charge distribution along the nascent peptide sequence by introducing mutations affects the rate of cotranslational folding, and the more positive the net charge of the protein, the deeper in the exit tunnel it is folded [42]. The combination of the tunnel geometry and electrostatic potential imposes restrictions that define the size, the complexity, and the timing of folding intermediates. Perturbations in the shape of the tunnel caused by deleting tunnel-exposed loops of 
uL23 and uL24 shift the onset of cotranslational protein folding, for example of proteins ADR1, R16, and I27 [43].

Nascent chains can interact with the peptide exit tunnel in specific ways that affect the rate of translation. Stretches of positively charged residues can slow down [44,45] or even stall [46] translation. Changes in translation rates can affect the rate of folding and the conformation of the resulting proteins $[47,48]$. Some peptides, such as those found in SecM, MifM, VemP, ErmCL, cause programmed translation arrest, thereby regulating the expression of the respective downstream genes [49]. These arrest peptides (AP) are usually $\sim 20$ amino acids long; they interact with the exit tunnel and distort the optimal geometry of the PTC [49,50]. In some cases, stalling brings into the PTC a pair of slowly reacting amino acids, such as proline and glycine that do not react with one another unless the active conformation of the PTC is induced. The AP of SecM is of particular interest [51-53]. When fully translated, the 17 amino acid SecM AP inhibits peptidyl transfer until an external force exerted on the nascent peptide alleviates stalling, allowing the ribosome to resume translation [53]. Cotranslational folding events can exert mechanical force of up to $8 \mathrm{pN}$ ) [54,55] and relieve AP stalling thereby allowing translation to continue. This is utilized in force-profile assays (FPA) to identify cotranslational folding events [51-53].

\section{Folding Inside the Exit Tunnel}

Early experiments using fluorescence resonance energy transfer (FRET) between labels attached at different positions in the nascent peptide suggested that transmembrane segments can form $\alpha$-helices within the exit tunnel in the proximity of the PTC [40]. Biochemical assays based on site-specific cysteine tagging (pegylation) of the nascent chain helped to establish that the secondary structure formation can happen in a tunnel zone proximal to the PTC or at the distal end of the tunnel [56-59]. Visualization of nascent chains by cryo-electron microscopy (cryo-EM) shows that $\alpha$-helices can form in the upper and lower regions of the tunnel [51,60-62], whereas the space at the constriction is too narrow to accommodate an $\alpha$-helix (Figure 3). However, not every polypeptide chain that ultimately adopts helical conformation starts folding inside the peptide exit tunnel. The overall hydrophobicity, propensity to form an $\alpha$-helix, and the element length are the major determinants of $\alpha$-helix formation within the tunnel [63]. Indeed, accessibility assays, FRET, and molecular dynamics simulations provide evidence that transmembrane helices favor early compaction during translation to a much larger extent than their soluble counterparts $[40,63,64]$.

Nascent chains can also form tertiary interactions within the exit tunnel of the ribosome $[59,65]$ and molecular dynamics simulations predicted a number of domain structures that could fold in the tunnel vestibule [66]. FPA reveals that small protein domains with a molecular weight $<10 \mathrm{kDa}$ (or $\leq 70$ amino acid residues) of various topologies encompassing $\alpha$-helices or $\beta$-sheets may fold within the first $80 \AA$ of the exit tunnel $[42,67]$. FPA and cryo-EM show that an entire Zn-finger domain of ADR1 folds into a native structure deep inside the exit tunnel of the ribosome [51] (Figure 4a). Also, the $\alpha$-helical $\mathrm{N}$-terminal domain of HemK forms a compact intermediate deep within the exit tunnel, although the native fold is attained only upon leaving the ribosome [37]. These examples also show that in some cases the tertiary interactions formed inside the tunnel can be very similar to the native structure of the isolated fully folded protein [51], whereas others are strictly cotranslational and not observed during protein refolding in solution [37]. The onset and trajectory of folding may be determined by the relative stability of the first accessible folding intermediate, rather than by the specific biophysical properties of the isolated native protein [16] (Figure $4 \mathrm{~b}$ ). 


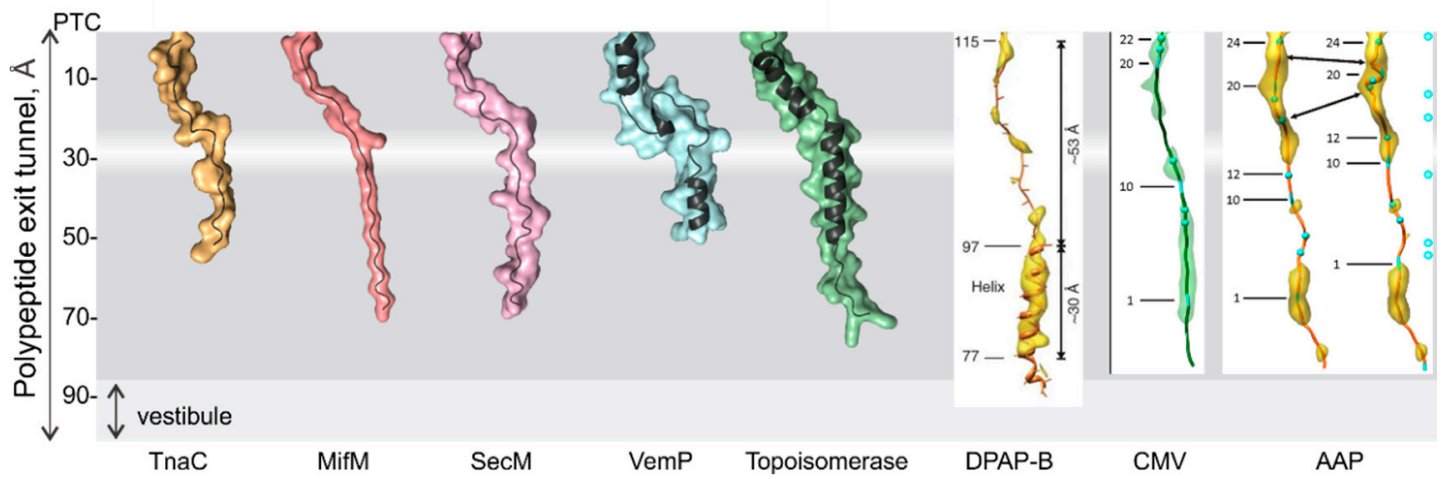

Figure 3. Examples of structures of nascent peptides in the polypeptide exit tunnel. Nascent peptides can interact with the tunnel walls as shown for TnaC [68], MifM [69], SecM [70], and CMV [71], or form $\alpha$-helices in the upper and lower regions of the tunnel, as illustrated for VemP [62] and DNA topoisomerase peptides [61]. An $\alpha$-helical structure of dipeptidylaminopeptidase B (DPAP-B) and the AAP peptide in the tunnel are also shown $[60,71]$. Structures shown on gray background are visualized using the PDB coordinates (PDB ID left to right: 4UY8; 3J9W; 3JBU; 5NWY; 5NP6). The coordinates of structures shown on white background are not available as PDB entries and are reproduced from the respective journals, with permission.

\section{Cotranslational Folding of Single Domain Proteins}

The ribosome can define a unique folding trajectory of single-domain proteins by inducing formation of simple folding units/intermediates early during translation. For example, a small globular $\mathrm{N}$-terminal domain of protein HemK that is a rapid two-state folder in solution undergoes gradual compaction on the ribosome $[37,72,73]$. Likewise, spectrin domain, which is a two-state folder in solution, begins to fold cotranslationally before the $\mathrm{C}$-terminus becomes available and proceeds via an ensemble of partially structured states $[16,74]$ (Figure $4 \mathrm{~b}$ ). Fluorescent proteins GFP and RFP cannot fold into the native state while the C-terminus is occluded by the ribosome, but the proteins remain in a compact folding-competent non-native conformation [75]. The nucleotide binding domain of cystic fibrosis transmembrane conductance regulator (CFTR) folds through a series of precisely timed and controlled nascent chain compaction events that are different from its folding trajectory in solution, which is facilitated by the ribosome through optimized translation kinetics [76]. The binding of its ligand, ATP, to the N-terminal domain stabilizes an energetically favorable local conformation, thereby contributing to the folding trajectory [77].

Somewhat surprisingly, also $\beta$-stranded domains can initiate folding on the ribosome via pathways that differ from those in solution. Upon cotranslational folding of the FLN5 filamin domain, the first intermediate is formed deep inside the exit tunnel, as found by FPA [73], although nuclear magnetic resonance (NMR) experiments suggest that this compaction is not identical to the final native fold and the protein appears unfolded. The protein then undergoes a transition to the native state after emerging from the exit tunnel $[78,79]$ (Figure 4c,d). Proteins containing repeat motifs can also fold sequentially. FPA reveals that a $\beta$-helix pentarepeat protein folds through at least four cotranslational intermediates, which are attributed to the stepwise compaction of the first several coils, followed by a compaction when the entire domain emerges from the exit tunnel [80]. In the cases where the ribosome induces early cotranslational folding, rapid initial compaction of the $\mathrm{N}$-terminal elements of the nascent chain can form the nucleus for further cotranslational folding. For complex domain topologies, the establishment of a stable folding nucleus on the ribosome ensures that a protein packs into conformations that do not lead to misfolding or aggregation [81]. Destabilization of the native domain in these cases does not change the onset of folding [16,81]. The folding trajectory is defined by the stability of folded or partially folded states formed on the ribosome, whereas the stability and folding rates of isolated native proteins are insufficient to predict the cotranslational folding pathway [16]. 
a

Cryo-EM: ADR1
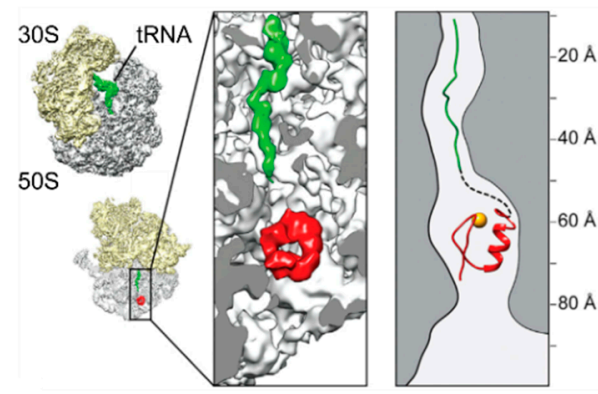

C

NMR: FLN5 and FLN6

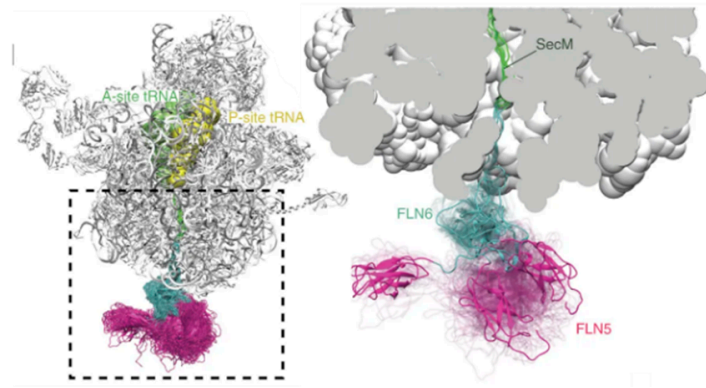

b

Cryo-EM: Spectrin

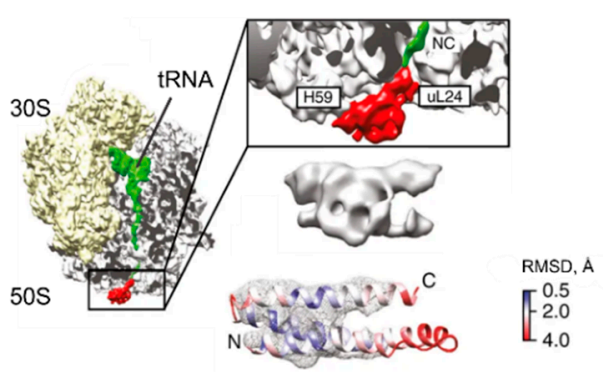

d NMR: FLN5
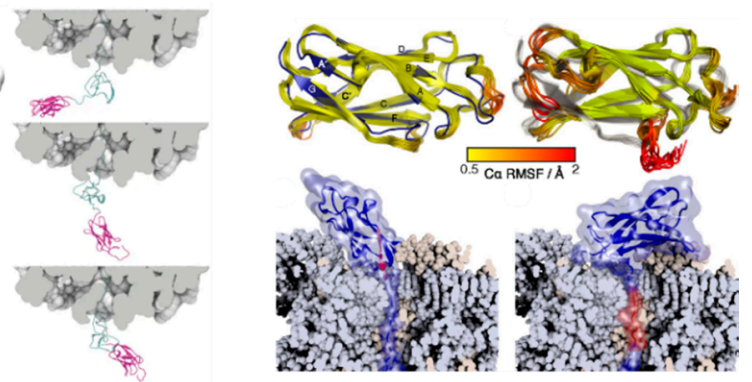

Figure 4. Tertiary structures of nascent peptides on the ribosome (adapted with permissions). (a) Cryo-electron microscopy (cryoEM) structure of the Zn-finger domain of ARD1 deep inside the exit tunnel of the ribosome. Figure adapted from [51]. (b) Cryo-EM structure of partially folded states of the spectrin domain at the exit tunnel vestibule, adapted from [16]. Root-mean-square deviation (RMSD) indicates the deviation of the native spectrin domain structure (PDB: 1AJ3) from the cryo-EM density map (EMD-3451) of the domain conformation at the ribosome surface. (c) Nuclear magnetic resonance (NMR) structures of the disordered FLN6 domain (cyan) and natively folded FLN5 (magenta), with representative conformations of FLN5 on the ribosome; figure adapted from [82]. (d) NMR structures of the native state (left) and an ensemble of intermediate states (right) for FLN5 on the ribosome [78].

There are also examples where the ribosome prevents folding until a large part of the domain emerges in the cytoplasm. Some small globular proteins that can rapidly refold from unfolded to native state in solution remain unfolded during translation and adopt their native-like assembly only when most of the peptide has emerged from the exit tunnel [83]. NMR studies of truncated SH3 peptides of various length show that on the ribosome they remain flexible and unstructured, but once the entire domain sequence emerges out of the tunnel, it folds into a compact, native-like $\beta$-sheet assembly [83]. Phi-value analysis [84], which allows one to estimate the contribution of each amino acid residue to the rate-limiting transition state on the protein folding pathway, suggests that the ribosome does not change the key contacts required for the transition towards the native structure of all $\beta$-sheet Ig domains of titin I27 [85] or SH3 domain [86]. In some cases the ribosome has no effect on folding. For example, the intrinsically disordered protein $\alpha$-synuclein is not perturbed on the ribosome, despite the interactions established between nascent protein and ribosome [82].

Cotranslational folding intermediates may have biological roles on their own. A structurally unique cotranslational intermediate of FtsY determines its targeting to the membrane [87]. During translation, a specific FtsY domain forms an extended helix that reorganizes into the final three-helix bundle only after the fully translated nascent chain is released from the ribosome. The extended conformation does not exist in the fully folded native protein, but is thought to facilitate the cotranslational localization of FtsY [87]. 
Coarse grained molecular dynamics simulations of co-translational folding for 5 different globular proteins suggest that the cooperativity of folding decreases on the ribosome due to the appearance of partially folded N-terminal intermediates that are not populated in solution. The ribosome decreases the diversity of the folding routes and increases the probability of folding beginning from the N-terminus [88]. Monte Carlo simulations of cotranslational folding processes suggest that during elongation the ribosome may support the progressive establishment of structures that are dominated by local interactions, whereas protein structures that are governed by more distal interactions do not fold until the nascent chain is released into solution [89]. Interestingly, there is some structural evidence that shows that truncated forms of a $\beta$-sheet protein in isolation may adopt an $\alpha$-helical conformation and undergo a conformational transition to the antiparallel $\beta$-sheet topology only when a sufficient length of the peptide chain is synthesized [90]. Although the latter work was carried out with protein fragments in solution, rather than with translating ribosomes, it points towards the idea that short-range interaction may be favored in early cotranslational intermediates, but as the peptide grows, the structure rearranges to establish the final long-range contacts. Interestingly, interaction with the translating ribosome may even coordinate the formation of the knot in the so-called knotted proteins [91,92]. Course grain simulations suggest that the nascent twisted loop sticks to the ribosome surface and is threated by the C-terminal part of the chain being pushed out of the ribosome, with the creation of the knot [91].

\section{Multidomain Protein Folding}

About $30-40 \%$ of proteins in prokaryotic and up to $75 \%$ in eukaryotic cells are multidomain proteins [93]. During refolding experiments in solution, multidomain proteins tend to misfold and form insoluble aggregates [94-96]. In the cell, the ribosome and the chaperones ensure the correct folding trajectory [97]. Systematic studies of protein coding sequences show that slowly translating codon clusters frequently occur at domain boundaries [30,48] suggesting that individual domains might be folding at least partly independently of one another starting from the N-terminal domain and proceeding in a vectorial fashion as each subsequent domain is synthesized. For example, the N-terminal domains of HemK and CFTR fold largely independently of the C-terminal part of the protein $[37,98]$. Similarly, in mammalian cells folding of multidomain fusion protein rapamycin binding protein (FRB)-GFP occurs co-translationally and strongly favors a domain-wise folding pathway [99].

There are only a few examples of multidomain proteins for which the cotranslational folding pathway is known. The only rigorously studied case is EF-G, a five domain translation factor that binds GTP. In isolation, EF-G refolds very inefficiently, both on the level of individual domains and of a complete protein, suggesting that the domains can form non-native off-pathway intermediates that preclude refolding to the native structure [100]. In contrast, in vivo EF-G folds all five of its domains into a functional conformation. On the ribosome, the N-terminal G domain of EF-G folds autonomously, but the nascent domain structure remains unstable [100], delaying folding until sufficient sequence information is available, or the subsequent domain/interaction partner becomes available for interaction. The folding of the G domain must occur before the folding of the next domain (domain II of EF-G). The ribosome can either accelerate or decelerate the folding of the G domain, compared to the rate of folding in solution, depending on how much amino acid sequence has been synthesized. The maximum folding rate of the nascent $G$ domain is achieved when the nascent chain comprises 386 amino acids; at shorter peptide lengths the ribosome decelerates $G$ domain folding, while for longer peptides the rates of folding on the ribosome are higher than in solution. The timing of the individual domain folding is crucial, because the interaction between the folded and unfolded domains in EF-G can result in unfolding of parts of the structure on the ribosome, thereby further complicating the cotranslational folding landscape [100,101]. In this case, the ribosome, together with trigger factor (TF), a cotranslationally acting chaperone, aids early folding steps to establish the correct path for folding [101]. Notably, in vivo folding of the eukaryotic homolog of EF-G, eEF2, requires the help of chaperones [102,103]. Interestingly, recent work on the cotranslational folding of domain III of EF-G 
shows that this domain is not stabilized by its N-terminal neighbors (domain G and domain II) and requires interactions with the C-terminal domains (domains IV and V) to adopt a stable structure [104]. This is probably related to the fact that domains $\mathrm{G}+\mathrm{II}$ and III+IV+V form the two superdomains of EF-G that move relative to each other during its function in translocation. These data also imply that about halfway through synthesizing EF-G, the folding pathway shifts from cotranslational to post-translational. The high degree of flexibility in domain III is required for EF-G to execute its function, but this feature leads to an increase in the number of unfolded domains during synthesis on the ribosome. This illustrates how different biological requirements have to be reconciled during protein biogenesis [104].

\section{The Ribosome Has a Destabilizing Effect on the Nascent Chain}

In addition to promoting correct protein folding, the ribosome prevents premature or incorrect folding. The surface of the ribosome destabilizes the nascent protein packing even after the proteins have fully emerged from the exit tunnel (Figure 1). The examples of the ribosome acting as a holdase are during the synthesis of FLN5 [82], RnaseH [105], T4 lysozyme [15], GFP and RFP [75]. Optical-tweezer experiments on the cotranslational folding of T4 lysozyme show that the nascent protein interacting with the ribosome surface has a different rate of folding than in solution. Changing the ionic strength of the buffer affects the protein folding rate on the ribosome, suggesting that electrostatic interactions between the peptide and the negatively charged ribosome surface are responsible for this effect [15]. In other cases, the ribosome can delay the formation of cotranslational intermediates at the emerging N-terminus, disfavor the formation of misfolded intermediates and increase the rate of their unfolding in order to maintain a folding-competent nascent polypeptide [106]. Delaying the compaction of nascent chains could be advantageous in ensuring that folding into stable conformations does not occur before the entire sequence is fully accessible. The fine-tuning of the folding window could be of particular importance for cotranslational folding of multidomain proteins, where interactions between unstable folding intermediates can derail folding of the entire protein [100,101]. The highly negative electrostatic charge of the ribosome surface may help in achieving these destabilizing effects. Modulating the net charge of an intrinsically disordered protein alters the population distribution of the dynamic nascent chain species on the ribosome: the higher the net negative charge of the nascent chain, the larger the fraction of the more dynamic population of the nascent chain on the ribosome [107]. Coarse-grained molecular dynamics simulations of several globular proteins attached to the ribosome by a linker of different length suggest that at the ribosome surface the entropy of the unfolded state increases and that of the native state decreases, causing destabilization of the nascent protein structure. The unfolding rates decrease and the folding rates increase linearly with the increasing linker length, which explains why native folds are stabilized as the protein moves away from the ribosome [88].

\section{Cotranslational Subunits Assembly}

Prokaryotic genomes are organized in operons where a single mRNA encodes multiple protein products. The individual subunits of protein complexes tend to be encoded within the same operon, and the order of genes in an operon is non-random and under selective evolutionary pressure [108]. Recent studies suggest that this may be maintained in part because the assembly of multisubunit proteins can begin cotranslationally. An elegant study employing the bacterial luciferase LuxAB shows that when both subunits are synthesized from a single bicistronic mRNA, LuxA binds to the nascent LuxB before the latter is released from the ribosome [109]. The extensive heterodimer interface between the two subunits is established as soon as the entire dimerization surface of LuxB emerges from the exit tunnel. Cotranslational assembly of multisubunit complexes is one of the most effective ways to ensure rapid and efficient recruitment of partner proteins in the crowded environment of the cell.

A significant fraction of eukaryotic proteins form large protein complexes [110]. mRNAs encoding the protein subunits of an oligomeric complex in eukaryotic cells are often colocalized [111], possibly to bring together interacting protein partners. However, even high local protein concentration cannot 
explain the high efficiency of protein complex assembly. Indeed some proteins are by themselves toxic to the cell [112] or unstable, intrinsically disordered and prone to aggregation [2,113]. Recent studies suggest that several mammalian nuclear transcription complexes assemble cotranslationally [114]. A systematic study of eukaryotic subunit assembly during translation by selective ribosome profiling shows that out of 12 hetero-oligomeric complexes studied, nine assembled cotranslationally and the remainder assembled with chaperone assistance [26]. In most cases, the uni-directionality of cotranslational assembly is evolutionarily preserved from prokaryotes to eukaryotes and the onset of subunit interaction coincides with the emergence of the interaction domain of the nascent peptide. Cotranslational association is favored in those cases where subunits are especially aggregation-prone [26]. Also yeast protein complexes, such as histone-modifying complexes methyl-transferase (SET1C) [115] and acetyltransferase (SAGA) assemble cotranslationally [116], as do cyclin protein complexes [117]. The ribosome may modulate the assembly of protein complexes by stabilizing individual protein domains or subunits [100] or adjusting the speed of translation [47] downstream of interaction domain boundaries [30]. This may help to find the optimal time window for interactions between the protein subunits. The electrostatic charge of the ribosomal surface can also act in regulating cotranslational subunit assembly. For example, intrinsically disordered proteins of opposite charge, ACTR and NCBD, form a complex on the ribosome cotranslationally, but only with ACTR as the nascent chain and NCBD free in solution, and not vice versa. The negatively charged nascent ACTR is repelled from the negatively charged ribosome surface and thus remains available for productive binding of its positively charged partner [118]. The repulsion of negatively-charged nascent chains is consistent with previous observation of increased dynamics of negatively charged nascent chains on the ribosome [107]. A picture emerges where cotranslational assembly of subunits depends on multiple features of proteins, ribosome and the cellular environment; it could thus be subject to regulation on multiple levels to maintain the proteostasis in the cell.

\section{Future Perspectives}

Recent work has shed new light on the mechanism and relevance of the cotranslational folding of nascent proteins. The major conclusion is that folding of many proteins is governed by the ribosome depending on the intrinsic properties of the nascent peptide, such as its type of fold, size, thermodynamic stability, surface charge, and function. Understanding the physico-chemical rules that govern cotranslational folding is one of the future challenges towards solving the folding problem. Because protein synthesis is an energetically costly process, the optimal interplay between translation kinetics and cotranslational folding can ensure efficient protein production. This makes the ribosome a key player in maintaining protein homeostasis in the cell, but also raises questions concerning the links between translation and folding. For example, folding has been suggested to affect the rate of translation, but experimental evidence for this is scarce. It is known that translation is a non-uniform process, but the reasons for ribosome pauses, the interaction between adjacent ribosomes in polysomes, and the understanding of the role of these translational events for protein folding has just started to emerge. To be able to make generalizations, we need more examples of how multidomain and oligomeric proteins fold. One puzzling question is how the ribosomes synthesizing the subunits of a multidomain complex colocalize to start the cotranslational assembly. Growing evidence suggests that the ribosome acts as a holdase for nascent proteins. However, it is unclear how the interactions of the downstream effectors, such as chaperones and protein biogenesis factors, shape the nascent protein-folding trajectory. One can expect that this will be among the major future questions for the years to come. 
Author Contributions: All authors contributed to the writing of the manuscript. All authors have read and agreed to the published version of the manuscript.

Funding: This research was funded by the Max Planck Society and by the European Research Council (ERC) Advanced Investigator Grant RIBOFOLD (proposal number $n^{\circ} 787926$ ).

Acknowledgments: We thank Wolfgang Wintermeyer for critical reading the manuscript.

Conflicts of Interest: The authors declare no conflict of interest.

\section{References}

1. Hartl, F.U. Protein misfolding diseases. Annu. Rev. Biochem. 2017, 86, 21-26. [CrossRef]

2. Sweeney, P.; Park, H.; Baumann, M.; Dunlop, J.; Frydman, J.; Kopito, R.; McCampbell, A.; Leblanc, G.; Venkateswaran, A.; Nurmi, A.; et al. Protein misfolding in neurodegenerative diseases: Implications and strategies. Transl. Neurodegener. 2017, 6, 6. [CrossRef]

3. Miller, S.B.; Mogk, A.; Bukau, B. Spatially organized aggregation of misfolded proteins as cellular stress defense strategy. J. Mol. Biol. 2015, 427, 1564-1574. [CrossRef] [PubMed]

4. Cutting, G.R. Cystic fibrosis genetics: From molecular understanding to clinical application. Nat. Rev. Genet 2015, 16, 45-56. [CrossRef] [PubMed]

5. Eaton, W.A.; Hofrichter, J. Sickle cell hemoglobin polymerization. Adv. Protein Chem. 1990, 40, 63-279. [CrossRef] [PubMed]

6. Santana, A.; Waiswo, M. The genetic and molecular basis of congenital cataract. Arq. Bras. Oftalmol. 2011, 74, 136-142. [CrossRef]

7. Labbadia, J.; Morimoto, R.I. Huntington's disease: Underlying molecular mechanisms and emerging concepts. Trends Biochem. Sci. 2013, 38, 378-385. [CrossRef]

8. Athanasiou, D.; Aguila, M.; Bellingham, J.; Li, W.; McCulley, C.; Reeves, P.J.; Cheetham, M.E. The molecular and cellular basis of rhodopsin retinitis pigmentosa reveals potential strategies for therapy. Prog. Retin. Eye Res. 2018, 62, 1-23. [CrossRef]

9. Ciryam, P.; Morimoto, R.I.; Vendruscolo, M.; Dobson, C.M.; O’Brien, E.P. In vivo translation rates can substantially delay the cotranslational folding of the Escherichia coli cytosolic proteome. Proc. Natl. Acad. Sci. USA 2013, 110, E132-E140. [CrossRef]

10. Young, R.; Bremer, H. Polypeptide-chain-elongation rate in Escherichia coli B/r as a function of growth rate. Biochem. J. 1976, 160, 185-194. [CrossRef]

11. Bostrom, K.; Wettesten, M.; Boren, J.; Bondjers, G.; Wiklund, O.; Olofsson, S.O. Pulse-chase studies of the synthesis and intracellular transport of apolipoprotein B-100 in Hep G2 cells. J. Biol. Chem. 1986, 261, 13800-13806. [PubMed]

12. Ingolia, N.T.; Lareau, L.F.; Weissman, J.S. Ribosome profiling of mouse embryonic stem cells reveals the complexity and dynamics of mammalian proteomes. Cell 2011, 147, 789-802. [CrossRef]

13. Garbuzynskiy, S.O.; Ivankov, D.N.; Bogatyreva, N.S.; Finkelstein, A.V. Golden triangle for folding rates of globular proteins. Proc. Natl. Acad. Sci. USA 2013, 110, 147-150. [CrossRef] [PubMed]

14. O’Brien, E.P.; Ciryam, P.; Vendruscolo, M.; Dobson, C.M. Understanding the influence of codon translation rates on cotranslational protein folding. Acc. Chem. Res. 2014, 47, 1536-1544. [CrossRef] [PubMed]

15. Kaiser, C.M.; Goldman, D.H.; Chodera, J.D.; Tinoco, I., Jr.; Bustamante, C. The ribosome modulates nascent protein folding. Science 2011, 334, 1723-1727. [CrossRef]

16. Nilsson, O.B.; Nickson, A.A.; Hollins, J.J.; Wickles, S.; Steward, A.; Beckmann, R.; von Heijne, G.; Clarke, J. Cotranslational folding of spectrin domains via partially structured states. Nat. Struct. Mol. Biol. 2017, 24, 221-225. [CrossRef]

17. Nissen, P.; Hansen, J.; Ban, N.; Moore, P.B.; Steitz, T.A. The structural basis of ribosome activity in peptide bond synthesis. Science 2000, 289, 920-930. [CrossRef]

18. Schwarz, A.; Beck, M. The benefits of cotranslational assembly: A structural perspective. Trends Cell Biol. 2019, 29, 791-803. [CrossRef] [PubMed]

19. Anfinsen, C.B. Principles that govern folding of protein chains. Science 1973, 181, 223-230. [CrossRef] [PubMed] 
20. Frydman, J.; Erdjument-Bromage, H.; Tempst, P.; Hartl, F.U. Co-translational domain folding as the structural basis for the rapid de novo folding of firefly luciferase. Nat. Struct. Biol. 1999, 6, 697-705. [CrossRef] [PubMed]

21. Samelson, A.J.; Bolin, E.; Costello, S.M.; Sharma, A.K.; O’Brien, E.P.; Marqusee, S. Kinetic and structural comparison of a protein's cotranslational folding and refolding pathways. Sci. Adv. 2018, 4, eaas9098. [CrossRef] [PubMed]

22. Evans, M.S.; Sander, I.M.; Clark, P.L. Cotranslational folding promotes beta-helix formation and avoids aggregation in vivo. J. Mol. Biol. 2008, 383, 683-692. [CrossRef]

23. Svetlov, M.S.; Kommer, A.; Kolb, V.A.; Spirin, A.S. Effective cotranslational folding of firefly luciferase without chaperones of the Hsp70 family. Protein Sci. 2006, 15, 242-247. [CrossRef]

24. Kolb, V.A.; Makeyev, E.V.; Spirin, A.S. Folding of firefly luciferase during translation in a cell-free System. EMBO J. 1994, 13, 3631-3637. [CrossRef] [PubMed]

25. Kolb, V.A.; Makeyev, E.V.; Spirin, A.S. Co-translational folding of an eukaryotic multidomain protein in a prokaryotic translation system. J. Biol. Chem. 2000, 275, 16597-16601. [CrossRef] [PubMed]

26. Shiber, A.; Doring, K.; Friedrich, U.; Klann, K.; Merker, D.; Zedan, M.; Tippmann, F.; Kramer, G.; Bukau, B. Cotranslational assembly of protein complexes in eukaryotes revealed by ribosome profiling. Nature 2018, 561, 268-272. [CrossRef]

27. Stein, K.C.; Frydman, J. The stop-and-go traffic regulating protein biogenesis: How translation kinetics controls proteostasis. J. Biol. Chem. 2019, 294, 2076-2084. [CrossRef]

28. Collart, M.A.; Weiss, B. Ribosome pausing, a dangerous necessity for co-translational events. Nucleic Acids Res. 2019. [CrossRef]

29. Kramer, G.; Shiber, A.; Bukau, B. Mechanisms of cotranslational maturation of newly synthesized proteins. Annu Rev. Biochem. 2019, 88, 337-364. [CrossRef]

30. Komar, A.A. A pause for thought along the co-translational folding pathway. Trends Biochem. Sci. 2009, 34, 16-24. [CrossRef]

31. Voss, N.R.; Gerstein, M.; Steitz, T.A.; Moore, P.B. The geometry of the ribosomal polypeptide exit tunnel. J. Mol. Biol. 2006, 360, 893-906. [CrossRef]

32. Selmer, M.; Dunham, C.M.; Murphy, F.V.T.; Weixlbaumer, A.; Petry, S.; Kelley, A.C.; Weir, J.R.; Ramakrishnan, V. Structure of the $70 S$ ribosome complexed with mRNA and tRNA. Science 2006, 313, 1935-1942. [CrossRef] [PubMed]

33. Schuwirth, B.S.; Borovinskaya, M.A.; Hau, C.W.; Zhang, W.; Vila-Sanjurjo, A.; Holton, J.M.; Cate, J.H. Structures of the bacterial ribosome at $3.5 \AA$ resolution. Science 2005, 310, 827-834. [CrossRef] [PubMed]

34. Dao Duc, K.; Batra, S.S.; Bhattacharya, N.; Cate, J.H.D.; Song, Y.S. Differences in the path to exit the ribosome across the three domains of life. Nucleic Acids Res. 2019, 47, 4198-4210. [CrossRef] [PubMed]

35. Malkin, L.I.; Rich, A. Partial resistance of nascent polypeptide chains to proteolytic digestion due to ribosomal shielding. J. Mol. Biol. 1967, 26, 329-346. [CrossRef]

36. Blobel, G.; Sabatini, D.D. Controlled proteolysis of nascent polypeptides in rat liver cell fractions. I. Location of the polypeptides within ribosomes. J. Cell Biol. 1970, 45, 130-145. [CrossRef]

37. Holtkamp, W.; Kokic, G.; Jager, M.; Mittelstaet, J.; Komar, A.A.; Rodnina, M.V. Cotranslational protein folding on the ribosome monitored in real time. Science 2015, 350, 1104-1107. [CrossRef]

38. Lu, J.; Kobertz, W.R.; Deutsch, C. Mapping the electrostatic potential within the ribosomal exit tunnel. J. Mol. Biol. 2007, 371, 1378-1391. [CrossRef]

39. Lucent, D.; Snow, C.D.; Aitken, C.E.; Pande, V.S. Non-bulk-like solvent behavior in the ribosome exit tunnel. PLoS Comput. Biol. 2010, 6, e1000963. [CrossRef]

40. Woolhead, C.A.; McCormick, P.J.; Johnson, A.E. Nascent membrane and secretory proteins differ in FRET-detected folding far inside the ribosome and in their exposure to ribosomal proteins. Cell 2004, 116, 725-736. [CrossRef]

41. Nissley, D.A.; O'Brien, E.P. Structural origins of FRET-observed nascent chain compaction on the ribosome. J. Phys. Chem. B 2018, 122, 9927-9937. [CrossRef] [PubMed]

42. Farias-Rico, J.A.; Ruud Selin, F.; Myronidi, I.; Fruhauf, M.; von Heijne, G. Effects of protein size, thermodynamic stability, and net charge on cotranslational folding on the ribosome. Proc. Natl. Acad. Sci. USA 2018, 115, E9280-E9287. [CrossRef] [PubMed] 
43. Kudva, R.; Tian, P.; Pardo-Avila, F.; Carroni, M.; Best, R.; Bernstein, H.D.; von Heijne, G. The shape of the ribosome exit tunnel affects cotranslational protein folding. eLife 2018, 7, e36326. [CrossRef] [PubMed]

44. Charneski, C.A.; Hurst, L.D. Positively charged residues are the major determinants of ribosomal velocity. PLoS Biol. 2013, 11, e1001508. [CrossRef]

45. Requiao, R.D.; de Souza, H.J.; Rossetto, S.; Domitrovic, T.; Palhano, F.L. Increased ribosome density associated to positively charged residues is evident in ribosome profiling experiments performed in the absence of translation inhibitors. RNA Biol. 2016, 13, 561-568. [CrossRef]

46. Lu, J.; Deutsch, C. Electrostatics in the ribosomal tunnel modulate chain elongation rates. J. Mol. Biol. 2008, 384, 73-86. [CrossRef]

47. Zhang, G.; Hubalewska, M.; Ignatova, Z. Transient ribosomal attenuation coordinates protein synthesis and co-translational folding. Nat. Struct. Mol. Biol. 2009, 16, 274-280. [CrossRef]

48. Buhr, F.; Jha, S.; Thommen, M.; Mittelstaet, J.; Kutz, F.; Schwalbe, H.; Rodnina, M.V.; Komar, A.A. Synonymous codons direct cotranslational folding toward different protein conformations. Mol. Cell 2016, 61, 341-351. [CrossRef]

49. Ito, K.; Chiba, S. Arrest peptides: Cis-acting modulators of translation. Annu. Rev. Biochem. 2013, 82, 171-202. [CrossRef]

50. Wilson, D.N.; Arenz, S.; Beckmann, R. Translation regulation via nascent polypeptide-mediated ribosome stalling. Curr. Opin. Struct. Biol. 2016, 37, 123-133. [CrossRef]

51. Nilsson, O.B.; Hedman, R.; Marino, J.; Wickles, S.; Bischoff, L.; Johansson, M.; Muller-Lucks, A.; Trovato, F.; Puglisi, J.D.; O'Brien, E.P.; et al. Cotranslational protein folding inside the ribosome exit tunnel. Cell Rep. 2015, 12, 1533-1540. [CrossRef]

52. Goldman, D.H.; Kaiser, C.M.; Milin, A.; Righini, M.; Tinoco, I.; Bustamante, C. Mechanical force releases nascent chain-mediated ribosome arrest in vitro and in vivo. Science 2015, 348, 457-460. [CrossRef] [PubMed]

53. Ismail, N.; Hedman, R.; Schiller, N.; von Heijne, G. A biphasic pulling force acts on transmembrane helices during translocon-mediated membrane integration. Nat. Struct. Mol. Biol. 2012, 19, 1018-1022. [CrossRef] [PubMed]

54. Wruck, F.; Katranidis, A.; Nierhaus, K.H.; Buldt, G.; Hegner, M. Translation and folding of single proteins in real time. Proc. Natl. Acad. Sci. USA 2017, 114, E4399-E4407. [CrossRef] [PubMed]

55. Leininger, S.E.; Trovato, F.; Nissley, D.A.; O’Brien, E.P. Domain topology, stability, and translation speed determine mechanical force generation on the ribosome. Proc. Natl. Acad. Sci. USA 2019, 116, 5523-5532. [CrossRef] [PubMed]

56. Lu, J.; Deutsch, C. Folding zones inside the ribosomal exit tunnel. Nat. Struct. Mol. Biol. 2005, 12, 1123-1129. [CrossRef] [PubMed]

57. Lu, J.; Deutsch, C. Secondary structure formation of a transmembrane segment in Kv channels. Biochemistry 2005, 44, 8230-8243. [CrossRef] [PubMed]

58. Kosolapov, A.; Tu, L.; Wang, J.; Deutsch, C. Structure acquisition of the T1 domain of Kv1.3 during biogenesis. Neuron 2004, 44, 295-307. [CrossRef]

59. Tu, L.; Khanna, P.; Deutsch, C. Transmembrane segments form tertiary hairpins in the folding vestibule of the ribosome. J. Mol. Biol. 2014, 426, 185-198. [CrossRef]

60. Bhushan, S.; Gartmann, M.; Halic, M.; Armache, J.P.; Jarasch, A.; Mielke, T.; Berninghausen, O.; Wilson, D.N.; Beckmann, R. alpha-Helical nascent polypeptide chains visualized within distinct regions of the ribosomal exit tunnel. Nat. Struct. Mol. Biol. 2010, 17, 313-317. [CrossRef]

61. Agirrezabala, X.; Samatova, E.; Klimova, M.; Zamora, M.; Gil-Carton, D.; Rodnina, M.V.; Valle, M. Ribosome rearrangements at the onset of translational bypassing. Sci. Adv. 2017, 3, e1700147. [CrossRef] [PubMed]

62. Su, T.; Cheng, J.; Sohmen, D.; Hedman, R.; Berninghausen, O.; von Heijne, G.; Wilson, D.N.; Beckmann, R. The force-sensing peptide VemP employs extreme compaction and secondary structure formation to induce ribosomal stalling. Elife 2017, 6. [CrossRef] [PubMed]

63. Bano-Polo, M.; Baeza-Delgado, C.; Tamborero, S.; Hazel, A.; Grau, B.; Nilsson, I.; Whitley, P.; Gumbart, J.C.; von Heijne, G.; Mingarro, I. Transmembrane but not soluble helices fold inside the ribosome tunnel. Nat. Commun. 2018, 9, 5246. [CrossRef]

64. Lange, S.; Franks, W.T.; Rajagopalan, N.; Doring, K.; Geiger, M.A.; Linden, A.; van Rossum, B.J.; Kramer, G.; Bukau, B.; Oschkinat, H. Structural analysis of a signal peptide inside the ribosome tunnel by DNP MAS NMR. Sci. Adv. 2016, 2, e1600379. [CrossRef] [PubMed] 
65. Kosolapov, A.; Deutsch, C. Tertiary interactions within the ribosomal exit tunnel. Nat. Struct. Mol. Biol. 2009, 16, 405-411. [CrossRef] [PubMed]

66. O’Brien, E.P.; Hsu, S.T.; Christodoulou, J.; Vendruscolo, M.; Dobson, C.M. Transient tertiary structure formation within the ribosome exit port. J. Am. Chem. Soc. 2010, 132, 16928-16937. [CrossRef]

67. Marino, J.; von Heijne, G.; Beckmann, R. Small protein domains fold inside the ribosome exit tunnel. FEBS Lett. 2016, 590, 655-660. [CrossRef]

68. Bischoff, L.; Berninghausen, O.; Beckmann, R. Molecular basis for the ribosome functioning as an L-tryptophan sensor. Cell Rep. 2014, 9, 469-475. [CrossRef]

69. Sohmen, D.; Chiba, S.; Shimokawa-Chiba, N.; Innis, C.A.; Berninghausen, O.; Beckmann, R.; Ito, K.; Wilson, D.N. Structure of the Bacillus subtilis $70 \mathrm{~S}$ ribosome reveals the basis for species-specific stalling. Nat. Commun. 2015, 6, 6941. [CrossRef]

70. Zhang, J.; Pan, X.; Yan, K.; Sun, S.; Gao, N.; Sui, S.F. Mechanisms of ribosome stalling by SecM at multiple elongation steps. Elife 2015, 4. [CrossRef]

71. Bhushan, S.; Meyer, H.; Starosta, A.L.; Becker, T.; Mielke, T.; Berninghausen, O.; Sattler, M.; Wilson, D.N.; Beckmann, R. Structural basis for translational stalling by human cytomegalovirus and fungal arginine attenuator peptide. Mol. Cell 2010, 40, 138-146. [CrossRef] [PubMed]

72. Mercier, E.; Rodnina, M.V. Co-translational folding trajectory of the HemK helical domain. Biochemistry 2018, 57, 3460-3464. [CrossRef] [PubMed]

73. Kemp, G.; Kudva, R.; de la Rosa, A.; von Heijne, G. Force-profile analysis of the cotranslational folding of HemK and Filamin domains: Comparison of biochemical and biophysical folding assays. J. Mol. Biol. 2019, 431, 1308-1314. [CrossRef] [PubMed]

74. Scott, K.A.; Batey, S.; Hooton, K.A.; Clarke, J. The folding of spectrin domains I: Wild-type domains have the same stability but very different kinetic properties. J. Mol. Biol. 2004, 344, 195-205. [CrossRef] [PubMed]

75. Kelkar, D.A.; Khushoo, A.; Yang, Z.; Skach, W.R. Kinetic analysis of ribosome-bound fluorescent proteins reveals an early, stable, cotranslational folding intermediate. J. Biol. Chem. 2012, 287, 2568-2578. [CrossRef]

76. Kim, S.J.; Yoon, J.S.; Shishido, H.; Yang, Z.Y.; Rooney, L.A.; Barral, J.M.; Skach, W.R. Translational tuning optimizes nascent protein folding in cells. Science 2015, 348, 444-448. [CrossRef]

77. Khushoo, A.; Yang, Z.; Johnson, A.E.; Skach, W.R. Ligand-driven vectorial folding of ribosome-bound human CFTR NBD1. Mol. Cell 2011, 41, 682-692. [CrossRef]

78. Waudby, C.A.; Wlodarski, T.; Karyadi, M.E.; Cassaignau, A.M.E.; Chan, S.H.S.; Wentink, A.S.; Schmidt-Engler, J.M.; Camilloni, C.; Vendruscolo, M.; Cabrita, L.D.; et al. Systematic mapping of free energy landscapes of a growing filamin domain during biosynthesis. Proc. Natl. Acad. Sci. USA 2018, 115, 9744-9749. [CrossRef]

79. Cabrita, L.D.; Cassaignau, A.M.E.; Launay, H.M.M.; Waudby, C.A.; Wlodarski, T.; Camilloni, C.; Karyadi, M.E.; Robertson, A.L.; Wang, X.; Wentink, A.S.; et al. A structural ensemble of a ribosome-nascent chain complex during cotranslational protein folding. Nat. Struct. Mol. Biol. 2016, 23, 278-285. [CrossRef]

80. Notari, L.; Martinez-Carranza, M.; Farias-Rico, J.A.; Stenmark, P.; von Heijne, G. Cotranslational folding of a pentarepeat beta-helix protein. J. Mol. Biol. 2018, 430, 5196-5206. [CrossRef]

81. Marsden, A.P.; Hollins, J.J.; O’Neill, C.; Ryzhov, P.; Higson, S.; Mendonca, C.; Kwan, T.O.; Kwa, L.G.; Steward, A.; Clarke, J. Investigating the effect of chain connectivity on the folding of a beta-sheet protein on and off the ribosome. J. Mol. Biol. 2018, 430, 5207-5216. [CrossRef]

82. Deckert, A.; Waudby, C.A.; Wlodarski, T.; Wentink, A.S.; Wang, X.; Kirkpatrick, J.P.; Paton, J.F.; Camilloni, C.; Kukic, P.; Dobson, C.M.; et al. Structural characterization of the interaction of alpha-synuclein nascent chains with the ribosomal surface and trigger factor. Proc. Natl. Acad. Sci. USA 2016, 113, 5012-5017. [CrossRef]

83. Eichmann, C.; Preissler, S.; Riek, R.; Deuerling, E. Cotranslational structure acquisition of nascent polypeptides monitored by NMR spectroscopy. Proc. Natl. Acad. Sci. USA 2010, 107, 9111-9116. [CrossRef] [PubMed]

84. Fersht, A.R.; Sato, S. Phi-value analysis and the nature of protein-folding transition states. Proc. Natl. Acad. Sci. USA 2004, 101, 7976-7981. [CrossRef] [PubMed]

85. Tian, P.; Steward, A.; Kudva, R.; Su, T.; Shilling, P.J.; Nickson, A.A.; Hollins, J.J.; Beckmann, R.; von Heijne, G.; Clarke, J.; et al. Folding pathway of an Ig domain is conserved on and off the ribosome. Proc. Natl. Acad. Sci. USA 2018, 115, E11284-E11293. [CrossRef] [PubMed]

86. Guinn, E.J.; Tian, P.; Shin, M.; Best, R.B.; Marqusee, S. A small single-domain protein folds through the same pathway on and off the ribosome. Proc. Natl. Acad. Sci. USA 2018, 115, 12206-12211. [CrossRef] [PubMed] 
87. Karniel, A.; Mrusek, D.; Steinchen, W.; Dym, O.; Bange, G.; Bibi, E. Co-translational folding intermediate dictates membrane targeting of the signal recognition particle receptor. J. Mol. Biol. 2018, 430, 1607-1620. [CrossRef] [PubMed]

88. O'Brien, E.P.; Christodoulou, J.; Vendruscolo, M.; Dobson, C.M. New scenarios of protein folding can occur on the ribosome. J. Am. Chem. Soc. 2011, 133, 513-526. [CrossRef]

89. Krobath, H.; Shakhnovich, E.I.; Faisca, P.F. Structural and energetic determinants of co-translational folding. J. Chem. Phys. 2013, 138, 215101. [CrossRef]

90. Hanazono, Y.; Takeda, K.; Miki, K. Structural studies of the N-terminal fragments of the WW domain: Insights into co-translational folding of a beta-sheet protein. Sci. Rep. 2016, 6, 34654. [CrossRef]

91. Dabrowski-Tumanski, P.; Piejko, M.; Niewieczerzal, S.; Stasiak, A.; Sulkowska, J.I. Protein knotting by active threading of nascent polypeptide chain exiting from the ribosome exit channel. J. Phys. Chem. B 2018, 122, 11616-11625. [CrossRef] [PubMed]

92. Baiesi, M.; Orlandini, E.; Seno, F.; Trovato, A. Sequence and structural patterns detected in entangled proteins reveal the importance of co-translational folding. Sci. Rep. 2019, 9, 8426. [CrossRef] [PubMed]

93. Ekman, D.; Bjorklund, A.K.; Frey-Skott, J.; Elofsson, A. Multi-domain proteins in the three kingdoms of life: Orphan domains and other unassigned regions. J. Mol. Biol. 2005, 348, 231-243. [CrossRef] [PubMed]

94. Brockwell, D.J.; Radford, S.E. Intermediates: Ubiquitous species on folding energy landscapes? Curr. Opin. Struct. Biol. 2007, 17, 30-37. [CrossRef]

95. Chen, Y.; Ding, F.; Nie, H.; Serohijos, A.W.; Sharma, S.; Wilcox, K.C.; Yin, S.; Dokholyan, N.V. Protein folding: Then and now. Arch. Biochem. Biophys. 2008, 469, 4-19. [CrossRef]

96. Jahn, M.; Buchner, J.; Hugel, T.; Rief, M. Folding and assembly of the large molecular machine Hsp90 studied in single-molecule experiments. Proc. Natl. Acad. Sci. USA 2016, 113, 1232-1237. [CrossRef]

97. Balchin, D.; Hayer-Hartl, M.; Hartl, F.U. In vivo aspects of protein folding and quality control. Science 2016, 353, aac4354. [CrossRef]

98. Kleizen, B.; van Vlijmen, T.; de Jonge, H.R.; Braakman, I. Folding of CFTR is predominantly cotranslational. Mol. Cell 2005, 20, 277-287. [CrossRef]

99. Han, Y.; David, A.; Liu, B.; Magadan, J.G.; Bennink, J.R.; Yewdell, J.W.; Qian, S.B. Monitoring cotranslational protein folding in mammalian cells at codon resolution. Proc. Natl. Acad. Sci. USA 2012, 109, 12467-12472. [CrossRef]

100. Liu, K.; Rehfus, J.E.; Mattson, E.; Kaiser, C.M. The ribosome destabilizes native and non-native structures in a nascent multidomain protein. Protein Sci. 2017, 26, 1439-1451. [CrossRef]

101. Liu, K.; Maciuba, K.; Kaiser, C.M. The ribosome cooperates with a chaperone to guide multi-domain protein folding. Mol. Cell 2019, 74, 310-319. [CrossRef] [PubMed]

102. Schopf, F.H.; Huber, E.M.; Dodt, C.; Lopez, A.; Biebl, M.M.; Rutz, D.A.; Muhlhofer, M.; Richter, G.; Madl, T.; Sattler, M.; et al. The co-chaperone Cns1 and the recruiter protein Hgh1 link Hsp90 to translation elongation via chaperoning Elongation Factor 2. Mol. Cell 2019, 74, 73-87.e8. [CrossRef] [PubMed]

103. Monkemeyer, L.; Klaips, C.L.; Balchin, D.; Korner, R.; Hartl, F.U.; Bracher, A. Chaperone function of Hgh1 in the biogenesis of eukaryotic Elongation Factor 2. Mol. Cell 2019, 74, 88-100.e9. [CrossRef] [PubMed]

104. Liu, K.; Chen, X.; Kaiser, C.M. Energetic dependencies dictate folding mechanism in a complex protein. Proc. Natl. Acad. Sci. USA 2019. [CrossRef]

105. Samelson, A.J.; Jensen, M.K.; Soto, R.A.; Cate, J.H.; Marqusee, S. Quantitative determination of ribosome nascent chain stability. Proc. Natl. Acad. Sci. USA 2016, 113, 13402-13407. [CrossRef]

106. Alexander, L.M.; Goldman, D.H.; Wee, L.M.; Bustamante, C. Non-equilibrium dynamics of a nascent polypeptide during translation suppress its misfolding. Nat. Commun. 2019, 10, 2709. [CrossRef]

107. Knight, A.M.; Culviner, P.H.; Kurt-Yilmaz, N.; Zou, T.; Ozkan, S.B.; Cavagnero, S. Electrostatic effect of the ribosomal surface on nascent polypeptide dynamics. ACS Chem. Biol. 2013, 8, 1195-1204. [CrossRef]

108. Wells, J.N.; Bergendahl, L.T.; Marsh, J.A. Operon gene order is optimized for ordered protein complex assembly. Cell Rep. 2016, 14, 679-685. [CrossRef]

109. Shieh, Y.W.; Minguez, P.; Bork, P.; Auburger, J.J.; Guilbride, D.L.; Kramer, G.; Bukau, B. Operon structure and cotranslational subunit association direct protein assembly in bacteria. Science 2015, 350, 678-680. [CrossRef]

110. Benschop, J.J.; Brabers, N.; van Leenen, D.; Bakker, L.V.; van Deutekom, H.W.; van Berkum, N.L.; Apweiler, E.; Lijnzaad, P.; Holstege, F.C.; Kemmeren, P. A consensus of core protein complex compositions for Saccharomyces cerevisiae. Mol. Cell 2010, 38, 916-928. [CrossRef] 
111. Williams, N.K.; Dichtl, B. Co-translational control of protein complex formation: A fundamental pathway of cellular organization? Biochem. Soc. Trans. 2018, 46, 197-206. [CrossRef] [PubMed]

112. Unterholzner, S.J.; Poppenberger, B.; Rozhon, W. Toxin-antitoxin systems: Biology, identification, and application. Mob. Genet. Elements 2013, 3, e26219. [CrossRef] [PubMed]

113. Oldfield, C.J.; Dunker, A.K. Intrinsically disordered proteins and intrinsically disordered protein regions. Annu. Rev. Biochem. 2014, 83, 553-584. [CrossRef]

114. Kamenova, I.; Mukherjee, P.; Conic, S.; Mueller, F.; El-Saafin, F.; Bardot, P.; Garnier, J.M.; Dembele, D.; Capponi, S.; Timmers, H.T.M.; et al. Co-translational assembly of mammalian nuclear multisubunit complexes. Nat. Commun. 2019, 10, 1740. [CrossRef] [PubMed]

115. Halbach, A.; Zhang, H.; Wengi, A.; Jablonska, Z.; Gruber, I.M.; Halbeisen, R.E.; Dehe, P.M.; Kemmeren, P.; Holstege, F.; Geli, V.; et al. Cotranslational assembly of the yeast SET1C histone methyltransferase complex. EMBO J. 2009, 28, 2959-2970. [CrossRef] [PubMed]

116. Kassem, S.; Villanyi, Z.; Collart, M.A. Not5-dependent co-translational assembly of Ada2 and Spt20 is essential for functional integrity of SAGA. Nucleic Acids Res. 2017, 45, 1186-1199. [CrossRef]

117. Duncan, C.D.; Mata, J. Widespread cotranslational formation of protein complexes. PLoS Genet 2011, 7, e1002398. [CrossRef]

118. Marino, J.; Buholzer, K.J.; Zosel, F.; Nettels, D.; Schuler, B. Charge interactions can dominate coupled folding and binding on the ribosome. Biophys. J. 2018. [CrossRef]

(C) 2020 by the authors. Licensee MDPI, Basel, Switzerland. This article is an open access article distributed under the terms and conditions of the Creative Commons Attribution (CC BY) license (http://creativecommons.org/licenses/by/4.0/). 


\section{Scope and aims of thesis}

The aim of this thesis was to investigate the folding trajectory and the conformational dynamics of nascent chains as they emerge from the ribosome during translation.

Our model system was the $\alpha$-helical N-terminal domain (NTD) of an E. coli (N5)-glutamine methyltransferase protein HemK on the ribosome. We have chosen the NTD as a model protein because in solution it folds independently of the CTD and its folding on the ribosome differs dramatically from the two-state concerted folding of the free protein in solution (Holtkamp et al., 2015; Kemp et al., 2019; Mercier and Rodnina, 2018). The cotranslational folding pathway of HemK NTD is not known in detail.

- We applied the force profile assay to investigate the full cotranslational folding pathway of HemK NTD - from the formation of the first a-helix deep inside the peptide exit tunnel, until the entire domain leaves the ribosomal exit tunnel. We performed this using the wild-type (wt) NTD and a variant of destabilized hydrophobic core (4xA) (Holtkamp et al., 2015).

- We generated a series of ribosome nascent chain complexes with HemK NTD at various folding steps along its cotranslational folding trajectory, as well as nascent chain variants of diminishing stability. We employed PET-FCS and kinetic modelling to uncover the rates of conformational fluctuations of cotranslational folding intermediates, and were able to define the contribution of the ribosome in maintaining the stability of these compact structures.

The results presented here show the first detailed cotranslational folding pathway for a protein beginning from the $\mathrm{N}$-terminus until the formation of the complete domain. We identify a number of cotranslational intermediates and provide a tool to investigate the dynamic fluctuations of these cotranslational intermediates in the confined peptide exit tunnel as well as at the surface of the ribosome. This is the first insight into the dynamics of cotranslational intermediates. The methods outlined here can be employed to study folding pathways and dynamics of any protein on the ribosome. 


\section{SECTION 3 - Results}

\section{Sequential folding of HemK NTD on the ribosome.}

Native folded HemK NTD (72 amino acids (aa)) consists of five a-helices ( $\mathrm{H} 1$ to $\mathrm{H} 5$ ) arranged into a globular domain, identical to the NTD conformation in the full HemK protein (Yang et al., 2004) (Figure 10a). To map the cotranslational folding pathway, we performed high-resolution FPA covering aa 22 to 101 of HemK. We generated a construct that encodes aa 1 to 101 of HemK, which includes the NTD (aa 1-73) and part of the interdomain linker connecting the NTD to the C-terminal domain, followed by 17 codons for the SecM arrest peptide, and an additional sequence encoding 20 aa of protein CspA; the latter served as a reporter for high-tension events in the nascent chain. With the HemK101 construct, the resulting nascent chain (including the SecM peptide; 118 aa in total) is long enough for the entire NTD to emerge from the ribosome. At low force, the ribosome is stalled by the SecM arrest peptide, generating an arrested translation product (AR) (Nilsson et al., 2015). If folding of HemK exerts force, translation arrest is alleviated, resulting in a longer peptide, which we denote as full-length (FL) (Figure 10b). We then constructed a series of mRNAs where the HemK sequence is trimmed in steps of one or two codons from the 3' end of the HemK mRNA. We translated these mRNAs in a fully reconstituted in vitro translation system from $E$. coli. In this system, the ribosomes are synchronized at the translation initiation step and start translation simultaneously after mixing with elongation factors and aa-tRNAs. The experimental setup allows for a single round of translation on a given mRNA, thereby avoiding the potential desynchronization due to re-initiation. The translation products, AR and FL, are separated by SDS-PAGE. By analyzing the fraction of FL product formed, we identify hightension folding events (Figure 10c). 
a
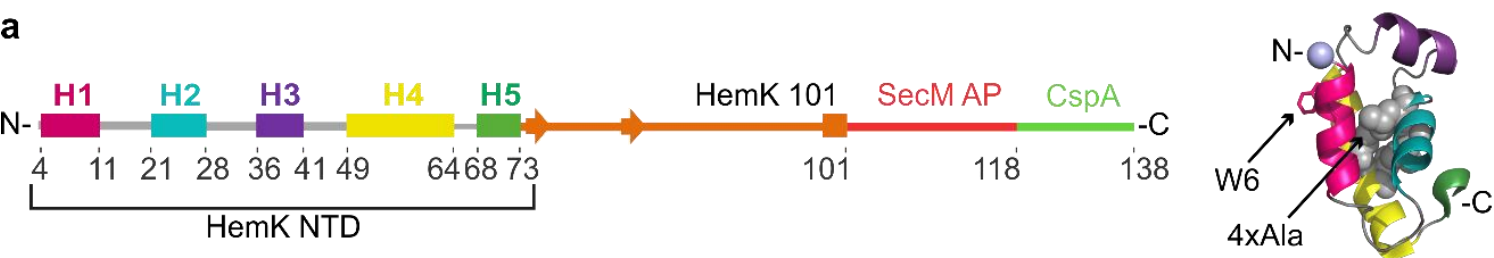

b

$\begin{array}{llllllllllllllllllllllll}\text { \#aa } & 22 & 24 & 26 & 28 & 30 & 32 & 34 & 36 & 38 & 40 & 42 & 44 & 46 & 48 & 50 & 52 & 54 & 56 & 58 & 62 & 64 & 66 & 68\end{array}$
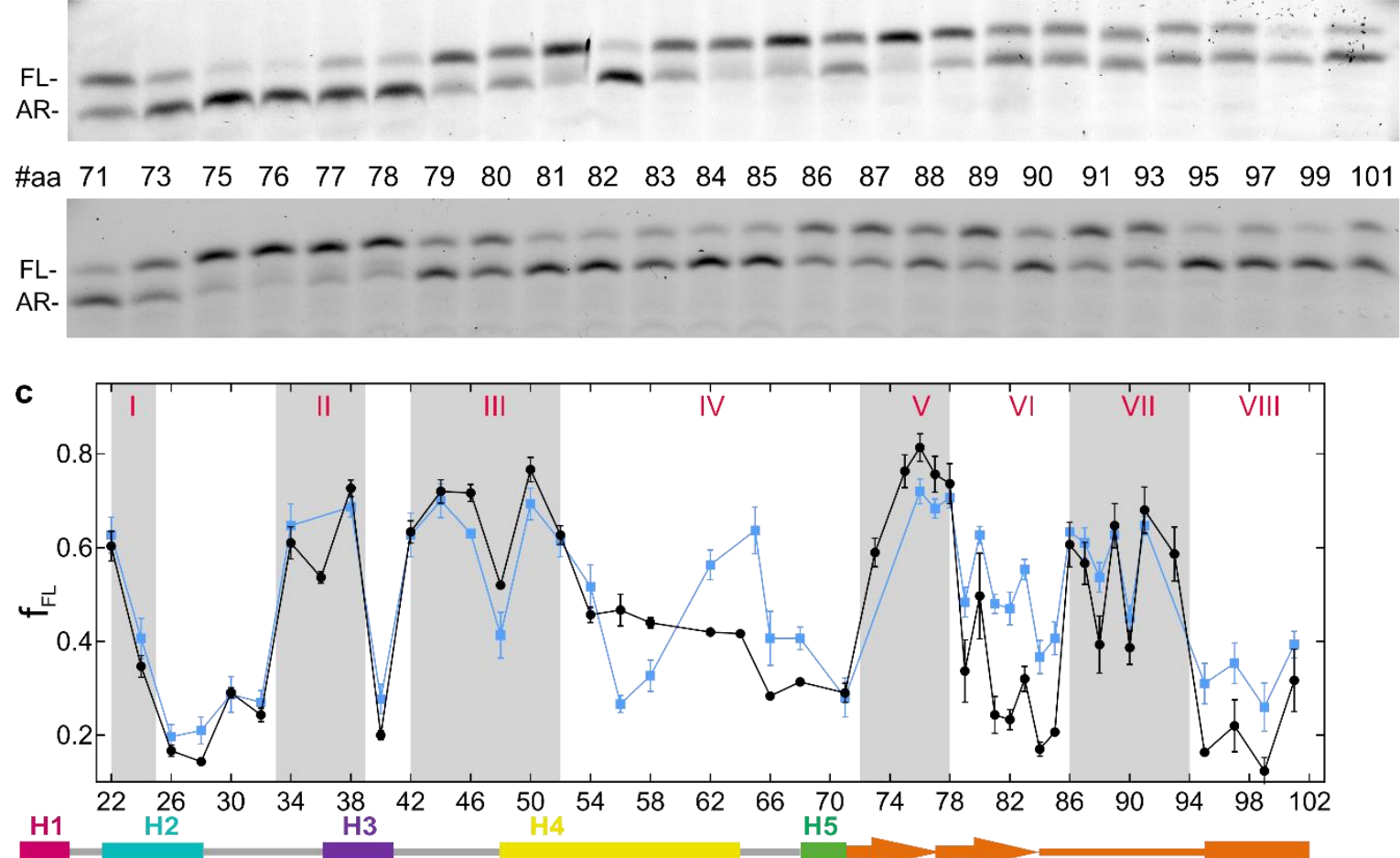

d

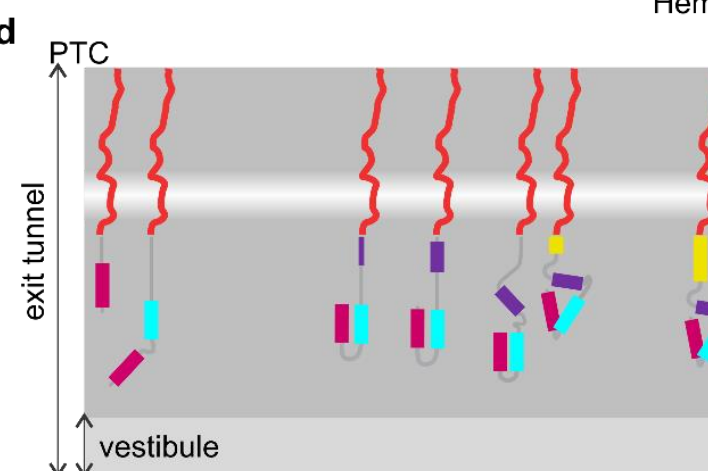

HemK length, aa

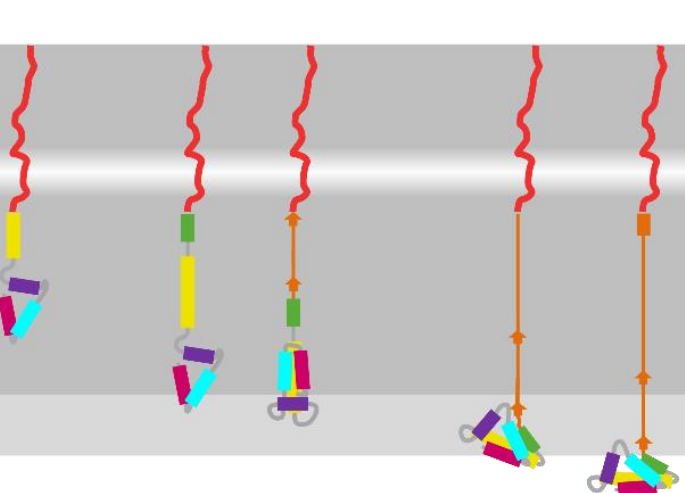

$\AA^{\mathbf{e}}$

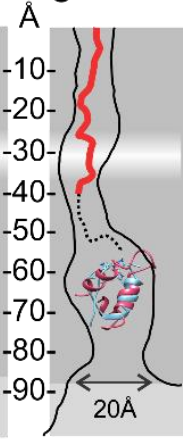

Figure 10 Co-translational folding of HemK NTD revealed by high-resolution FPA

a. Schematic representation of the FPA sensor. HemK NTD helices (H) 1-5, the C-terminal linker (orange), SecM arrest peptide (red), and CspA (green) (left) and crystal structure of HemK NTD (PDB ID: 1T43) (right). The mutations introduced in the 4xA variant (L27A, L28A, L55A, L58A) are shown in gray and the $\mathrm{N}$-terminal fluorophore position is shown in lilac.

b. SDS-PAGE of in vitro translation products for the FPA construct of wt HemK. The length of the nascent chain from the N-terminus to SecM AP is indicated (\#aa). FL, full-length product; AR, arrested peptide.

c. Force profile of HemK NTD folding. $f_{\mathrm{FL}}$ is the fraction of the full-length product formed during in vitro translation. Black, HemK wt; blue, HemK 4xA mutant; error bars indicate standard error of 
mean calculated from three independent biological replicates $(N=3)$. The schematic underneath shows the potential secondary structure of HemK at the indicated aa chain length.

d. Schematic diagram of HemK NTD compaction events during translation; color code as in (a). The constriction site is indicated by a white band.

e. Schematic overlay of the HemK H1-H3 crystal structure (pink) (PDB ID: 1T43) and ADR1 Zn finger domain crystal structure (green) inside the peptide exit tunnel, 60-80 $\AA$ from the PTC (Nilsson et al., 2015) (PDB ID: 2ADR; EM map: EMD-3079).

The detailed force profile of the HemK NTD reveals several distinct force-generating folding events starting early inside the exit tunnel until the entire NTD emerges from the ribosome (Figure 10c,d). The early high-tension regions are observed at nascent chain lengths of aa 2224 (I), 33-39 (II) and 42-52 (III) with a transient force relief at aa 48. At these chain lengths, the nascent peptide is likely to reside in the exit tunnel, as it is protected from protease digestion (Holtkamp et al., 2015). At 22-24 aa, the nascent peptide entails $\mathrm{H} 1$ and the subsequent loop region that moved past the constriction; hence, folding of $\mathrm{H} 1$ on its own or together with the first helical turn of $\mathrm{H} 2$ produce enough force to alleviate SecM stalling. This very early cotranslational intermediate of HemK folding deep inside the exit tunnel was not observed so far. The force level decreases as more of $\mathrm{H} 2$ emerges and $\mathrm{H} 1$ moves further down the exit tunnel, and then increases again before the complete helix $\mathrm{H} 3$ is synthesized. It is likely that once $\mathrm{H} 1$ and $\mathrm{H} 2$ move towards the more open space of the vestibule, they begin to form tertiary interactions, thereby generating tension.

Region III at aa 42-52 (Figure 10c,d) broadly coincides with the compacted intermediate identified by FRET and FPA studies (Holtkamp et al., 2015; Kemp et al., 2019; Mercier and Rodnina, 2018), except for the tension relief at 48 aa, which has not been observed before. The tension increases as the entire $\mathrm{H} 3$ emerges below the constriction and most likely corresponds to the formation of $\mathrm{H} 3$ and its docking onto the preceding two-helix structure. The transient tension relief at 48 amino acids may separate the helix formation and docking events. The $\mathrm{H} 1-\mathrm{H} 3$ intermediate structure is expected to reside approximately $50-60 \AA$ away from the PTC in the region within the exit tunnel that is known to accommodate a folded small zincfinger domain protein (ADR1) (Nilsson et al., 2015). To validate the feasibility of the HemK intermediate forming in this region of the tunnel we have superimposed the HemK $\mathrm{H} 1-\mathrm{H} 3$ onto the structure of the ADR1 domain in the tunnel (Nilsson et al., 2015). The two domains are of similar size (Figure 10e), and even if the HemK H1-H3 occupies a slightly larger volume than ADR1, it is very likely that the folding intermediate of $\mathrm{H} 1-\mathrm{H} 3$ can be accommodated in this region of the tunnel. The analysis of the folding regions I-III provides the first example of multiple cotranslational folding intermediates being resolved at such high precision inside the exit tunnel. 
As HemK becomes longer than 53 aa, the tension decreases to intermediate values, but still remains above the baseline tension level ( 0.2) (Figure 10c, region IV). Protease protection experiments suggest that when the nascent chain reaches the total length of 84 aa, position 34 (fluorescently labeled in those experiments) is no longer protected by the ribosome (Holtkamp et al., 2015). In the FPA experiments, this total chain length corresponds to 67 aa of HemK, of which at least 34 aa must be exposed to the solvent. At the HemK NTD length of 72 aa (99 aa total), part or all of $\mathrm{H} 4$ may emerge from the vestibule and be ready to initiate the formation of further hydrophobic interactions in the HemK NTD (Figure 10d). The next hightension intermediate at aa 72-78 (region V) forms when $\mathrm{H} 5$ and part of the following interdomain linker move past the constriction and $\mathrm{H} 4$ is further displaced towards the vestibule. A stable folding of $\mathrm{H} 4$ within the exit tunnel may form hydrophobic interactions with the $\mathrm{H} 1-\mathrm{H} 3$ structure, which generates the high-tension peak because the structure is closer to a narrow part of the vestibule. As the nascent chain grows, the $\mathrm{H} 1-\mathrm{H} 4$ intermediate again moves away from the exit port, which results in further structural rearrangements reflected in the decrease of tension interrupted by short force spikes at aa 80 and 83 (region $\mathrm{VI}$ ).

The final high-tension compaction (step VII) occurs when the length of the HemK NTD exceeds 86 aa, placing the entire NTD, $\mathrm{H} 1$ through $\mathrm{H} 5$, outside of the confines of the exit tunnel (Figure $10 \mathrm{c}, \mathrm{d}$ ). When NTD length is $>95$ aa (112 aa total peptide length), we observe only basal tension levels, indicating that the final cotranslational folding to a near-native conformation of the NTD occurred at step VII, consistent with the final steps of NTD cotranslational folding suggested previously (Holtkamp et al., 2015; Mercier and Rodnina, 2018).

In addition to the wt HemK NTD, we examined cotranslational folding of the destabilized HemK NTD variant $(4 \times A)$, where four conserved Leu residues of the hydrophobic core (Leu 27 and 28 in H2, and 55 and 58 in H4) were mutated to Ala (Figure 10a) (Holtkamp et al., 2015). The force profile inside the exit tunnel is identical for the wt and the 4XA variant (folding steps I-III), but as the peptide begins to emerge at the vestibule, the force profiles start to deviate (Figure 10c, Figure 11). As more of $\mathrm{H} 4$ becomes accessible for docking to the $\mathrm{H} 1-\mathrm{H} 3$ structure, Leuspecific interactions within the hydrophobic core start to matter. For the $4 \times A$ variant, we observe a drop in tension at a total length of 73 aa (56 aa of HemK NTD), and the appearance of a high-force intermediate at a chain length where the tension in the wt HemK NTD gradually decreases (aa 62-64) (Figure 10c, region IV). The change in folding at region IV could result from a delay (of $4 \mathrm{aa}$ ) in folding for the $4 \times \mathrm{A}$ variant compared to the wt. This could be because in the absence of the key Leu residues in the hydrophobic core a longer peptide is required to stabilize the packing, whereas the wt variant folds continuously as more of the nascent chain 
becomes available. Alternatively, it is possible that this peak indicates an intermediate state unique to $4 \times A$.

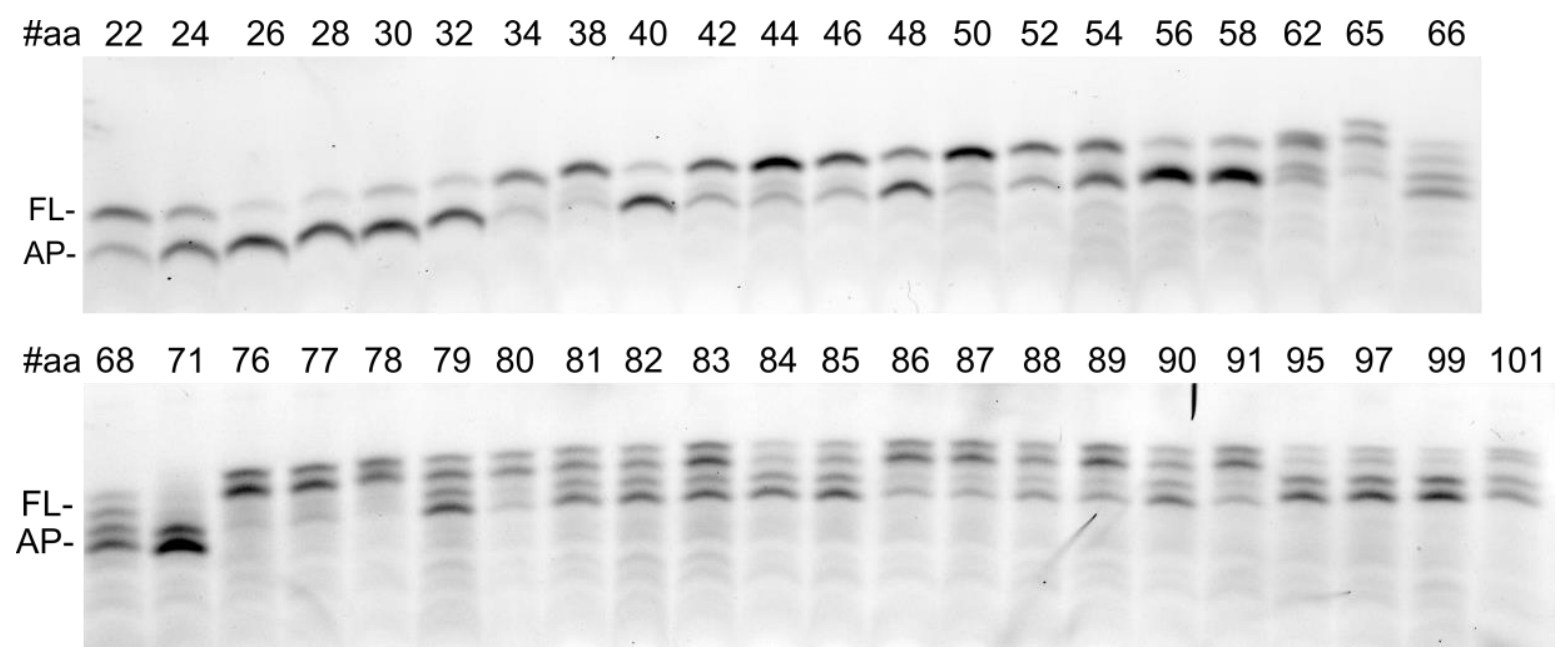

Figure 11 Representative SDS PAGE of FPA for HemK 4xA variant.

FL - full-length peptide, AP - arrested peptide, \#aa length of HemK 4xA construct in amino acids.

The two following cotranslational folding steps V and VII are similar in duration and amplitude for $4 \times A$ and wt, suggesting that these rearrangements are independent of the hydrophobic core packing. In contrast, in regions VI and VIII, the 4XA variant generates consistently higher tension than the wt NTD. This agrees with the notion that the 4xA variant adopts an expanded conformation; the timing of the discrete rearrangements that occur at step IV appears similar for 4xA and wt NTD, but each time when a more bulky 4xA peptide moves towards the ribosome surface, the tension increases. This difference in tension may indicate that the nonnative 4xA fold occupies a larger volume has a more dynamic structure, or there are changes in domain stability (Leininger et al., 2019).

\section{Nascent chain dynamics monitored with PET-FCS.}

Next, we monitored the dynamics or ribosome-bound nascent chains by PET-FCS. We utilized the native Trp6 residue (W6) in HemK that could quench an N-terminal ATTO655 fluorophore (Figure 10a) upon coming into van der Waals distance (Doose et al., 2009). The dynamic motions of the nascent chain define the frequency of these quenching interactions, and the resulting fluorescence intensity fluctuations can be used to track the internal dynamics of the nascent chain (Neuweiler et al., 2003a). 
First, we studied the dynamics of the HemK NTD in solution. We prepared a 70 aa-long NTD wt or 4XA by in vitro translation using an ATT0655-labeled initiator Met-tRNA ${ }^{\mathrm{fMet}}$, purified the complexes, and then chemically released the nascent chains from the ribosome (Methods). As a control for the PET signal, we also prepared NTD variants where Trp6 is replaced with nonquenching Phe (W6F). FCS measurements of the isolated NTD showed a stable and highly reproducible diffusion time of the peptide $\left(t_{d 1} \approx 6 \times 10^{-5} s\right)$ (Table 1$)$. However, no PET signal was observed, as the autocorrelation functions (ACF) with W6 and W6F proteins are identical for wt and 4xA constructs (Figure 12a). This result indicates that there are no dynamic fluctuations at the N-terminus of the HemK NTD in solution. To test this notion, we performed control measurements with a short HemK peptide of 14 aa, which should be unstructured in solution (Holtkamp et al., 2015) (Figure 12b). After testing different conditions, we were able to observe fast dynamics due to PET with a quenching relaxation time of $\sim 5 \times 10^{-7} \mathrm{~s}$ in the presence of glycerol (Figure 12b, Table 1). However, when the experiments with HemK NTD wt or 4xA are repeated at the same conditions, we observe only an increase in the diffusion time $\left(\mathrm{t}_{\mathrm{d} 1}=\sim 30 \times 10^{-5} \mathrm{~s}\right.$ ), but no PET signal corresponding to structural fluctuations (Figure 12c, Table 1). Thus, in solution, HemK NTD, as well as its destabilized $4 \times A$ variant, folds into a domain where $\mathrm{W} 6$ is not accessible for interaction with the $\mathrm{N}$-terminal dye.

a

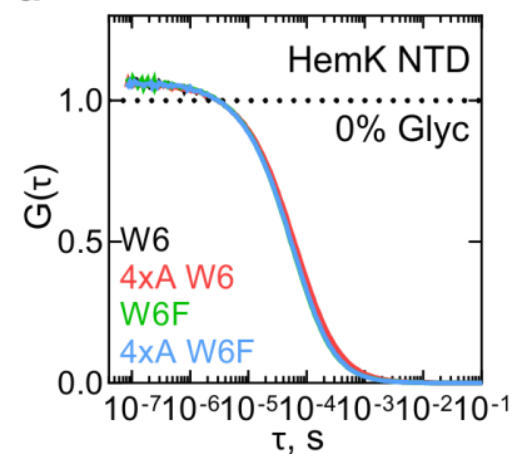

b

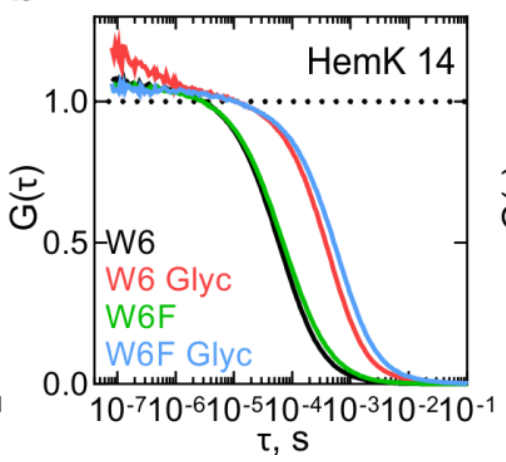

C

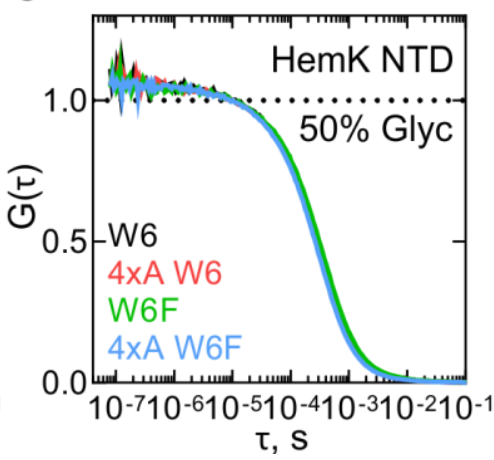

Figure 12 Monitoring dynamics of HemK constructs in solution by PET-FCS

a. ACF of the HemK NTD free in solution. Black, wt variant with Trp at position 6 (W6); red, destabilized 4XA HemK W6 variant; green, HemK wt with no Trp in the nascent chain (W6F); blue, 4xA HemK W6F variant; each curve is derived from two separate release experiments and each experiment consists of at least 4 technical replicates $(N \geq 8)$.

b. Autocorrelation curves of HemK14 peptide in solution. Black, W6 variant at low solvent viscosity; red, W6 at high viscosity in the presence of $50 \%$ glycerol (Glyc); green, W6F variant at low viscosity; blue, $\mathrm{W} 6 \mathrm{~F}$ variant at high viscosity. Shown are representative curves of at least two experimental repeats, each consisting of at least 4 technical replicates $(N \geq 8)$.

c. Autocorrelation curves of HemK NTD released from the ribosome at high viscosity conditions (50\% glycerol). Black, wt W6; red, 4xA W6; green, wt W6F; blue, 4xA W6F. Each curve is derived from two separate release experiments and each experiment consists of at least 4 technical replicates $(N \geq 8)$. 
Table 1 ACF fits of HemK constructs in solution.

Each ACF comprised of average of $\mathrm{N} \geq 8$. All fit errors are calculated as standard error of the mean and are $<10 \%$. Rates $k_{1}$ and $k_{d}$ are in $s^{-1} . \tau_{1}$ and $\tau_{d 1}$ are relaxation times of the respective exponents, in $\mathrm{s}, \tau=1 / \mathrm{k}$.

\begin{tabular}{|c|c|c|c|c|c|c|}
\hline HemK & $\mathrm{c}_{1}$ & $\tau_{1} \times 10^{-7}$ & $\mathrm{k}_{1} \times 10^{6}$ & $\mathrm{~N}$ & $\tau_{\mathrm{d} 1} \times 10^{-5}$ & $\mathrm{k}_{\mathrm{d}} \times 10^{3}$ \\
\hline 0\% glycerol & & & & & & \\
\hline 70 W6 & & & & 0.83 & 5.16 & 19.38 \\
\hline 70 W6F & & & & 0.83 & 5.42 & 18.43 \\
\hline 70 4xA W6 & & & & 0.85 & 5.78 & 17.30 \\
\hline 70 4xA W6F & & & & 0.83 & 5.59 & 17.89 \\
\hline 14 W6 & & & & 0.84 & 5.88 & 17.00 \\
\hline 14 W6F & & & & 0.86 & 6.61 & 15.13 \\
\hline 50\% glycerol & & & & & & \\
\hline 70 W6 & & & & 0.94 & 30.78 & 3.25 \\
\hline 70 W6F & & & & 0.95 & 33.87 & 2.95 \\
\hline $704 \times A ~ W 6$ & & & & 0.95 & 30.40 & 3.29 \\
\hline $704 \times A ~ W 6 F$ & & & & 0.95 & 29.10 & 3.44 \\
\hline 14 W6 & 0.15 & 5.13 & 1.95 & 0.97 & 45.09 & 2.22 \\
\hline 14 W6F & & & & 0.97 & 60.31 & 1.66 \\
\hline
\end{tabular}

We next applied PET-FCS to study dynamics of nascent peptides. We generated RNCs at three stages of translation based on the FRET and PET measurements (Mercier and Rodnina, 2018) (Figure 13a). To monitor the nascent chain dynamics during the compaction of $\mathrm{H} 1-\mathrm{H} 3$ within the exit tunnel, we used RNC with a 70 aa-long nascent chain (HemK 70), which shows a high FRET signal and maps roughly between FPA regions III and IV, given slight uncertainty in the positioning of the C-terminal 17 aa of native HemK compared to SecM (Figure 10c,d). An RNC with the 102 aa-long nascent chain (HemK 102) should expose $\mathrm{H} 1-\mathrm{H} 4$ at the ribosome surface and maps onto the end of FPA region VI; earlier PET measurements indicate a folding transition around this chain length (Mercier and Rodnina, 2018). To monitor the fully emerged domain, we used an RNC with a 112 aa-long nascent chain (FPA region VIII; Figure 10c,d).

To understand how destabilization of the hydrophobic core of the NTD affects the nascentchain dynamics, we also used the respective 4xA variants. One complication of the PET-FCS experiments with ribosome complexes is that the Trp residues in ribosomal proteins and guanines of the rRNA also quench the N-terminal fluorophore (Doose et al., 2009). To account for these interactions of the nascent peptide with the ribosome surface, we compare the W6 and the respective W6F control constructs (Figure 13b-d). 
a

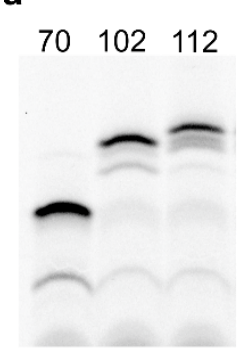

b

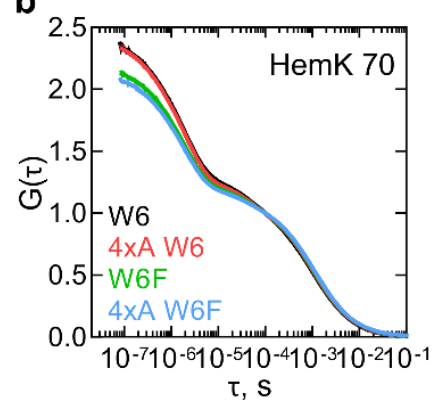

C

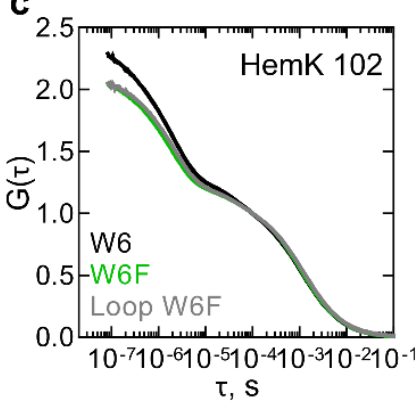

d

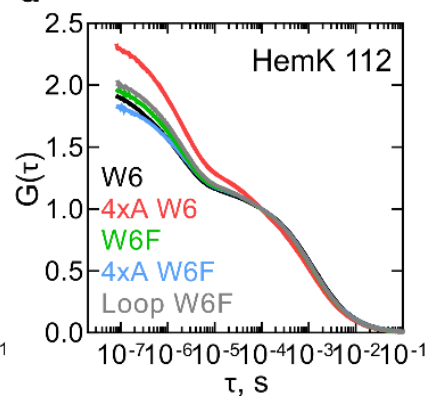

Figure 13 Dynamics of HemK on the ribosome monitored by PET-FCS

a. SDS PAGE of nascent chains produced by in vitro translation visualized after RNC purification using the fluorescence of ATTO655. The aa length of HemK wt constructs is indicated.

b. Autocorrelation curves for HemK 70-RNC. Black, wt W6; red, 4xA W6; green, wt W6F; blue, $4 \times A$ W6F. Each curve is derived from at least two separate RNC preparations and each experiment consists of at least 4 technical replicates $(N \geq 8)$.

c. Autocorrelation curves for HemK 102-RNC. Black, wt W6, green, wt W6F; grey, W6F variant with loop extension between helix 3 and helix 4 . Each curve is derived from at least two separate RNC preparations and each experiment consists of at least 4 technical replicates $(N \geq 8)$.

d. Autocorrelation curves for HemK 112-RNC. Black, wt W6; red, 4xA W6; green, wt W6F; blue, 4xA W6F; grey, W6F variant with a loop extension between helix 3 and helix 4. Each curve is derived from a minimum of two separate RNC preparations and each experiment consists of at least 4 technical replicates $(N \geq 8)$.

Previous published work showed that HemK W6F variants are translated with the same average translation rate and undergo similar compaction events on the ribosome when comparing to HemK wt (Holtkamp et al., 2015). Even though the purified W6F has a lower melting temperature compared to the wt, at $37^{\circ} \mathrm{C}$ this variant is still fully folded. Using FPA assay we additionally confirmed that the cotranslational folding trajectory of HemK W6F is identical compared to the wt (Figure 14). 


\section{a}

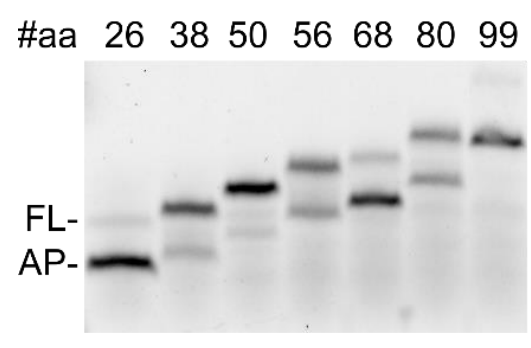

b

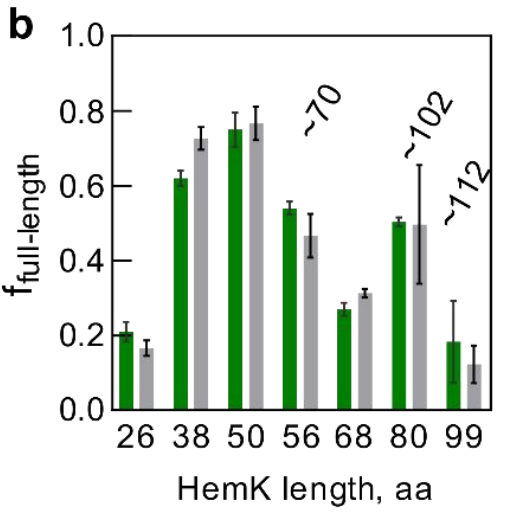

\section{Figure 14 FPA of HemK W6F}

a. A representative gel with selected force profile constructs \#aa - length of HemK nascent chain in number of amino acids;

b. Graph shows the fraction of full-length product generated at each construct length in green W6F, in grey wt. Approximate RNC 70, 102 and 112 positions are indicated on the profile.

For all RNCs tested, PET-FCS experiments yield multiphasic ACFs spanning the timescale from ms to ns. The diffusion time of the RNC is in the ms time range (Samelson et al., 2016). Initial exponential fitting reveals at least three fast dynamic components in the ns to $\mu$ s time range (Figure 13, Table 2). One of the components could reflect the triplet state pumping and relaxation of the ATTO655 dye in the complex with the ribosome. We measured the same RNC with increasing laser power to determine whether any of the exponents originate from the triplet relaxation (Figure 15a). We found that the third exponent amplitude changed depending on the laser power (Figure 15b), indicating that the ATTO655 triplet state relaxation time on the ribosome is $40 \mu \mathrm{s}$. This is somewhat larger than that measured with a model peptide, which is in the range of $2 \mu$ s (Luitz et al., 2017). The increase of the triplet state lifetime on the ribosome is most likely due to a more restricted conformational space on the ribosome rather than for a peptide diffusing in solution. The ribosome can lock the dye in a particular orientation and chemical environment extending the triplet lifetime (Saviotti and Galley, 1974). 
a

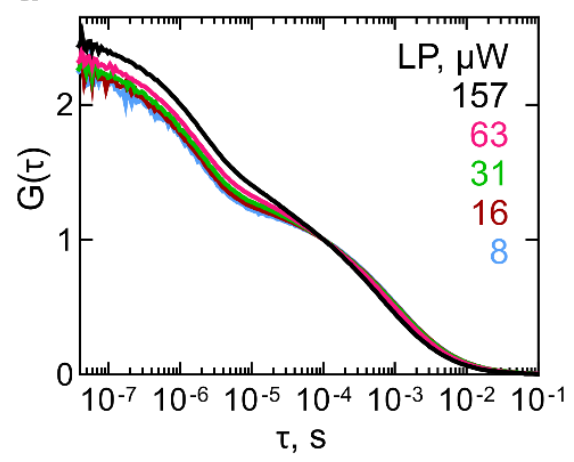

b

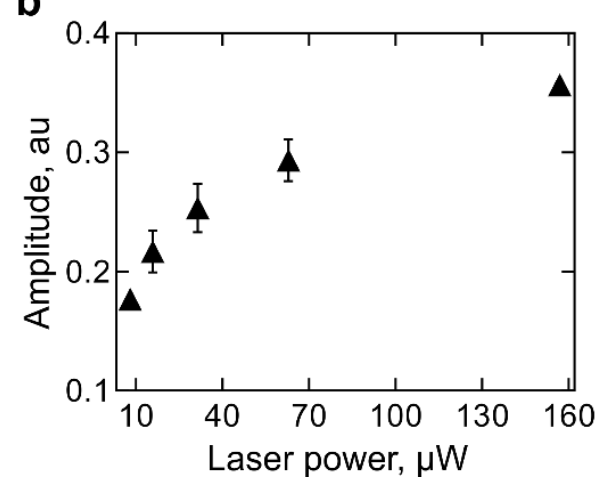

Figure 15 ATTO 655 triplet state in RNC

a. Autocorrelation curves of HemK 102 wt RNC measurements with increasing laser power (LP); each curve is an average $\mathrm{N} \geq 8$.

b. Amplitude of the triplet state relaxation time.

We then fitted the ACF curves (Figure 13b-d) using a combination of two exponential decays, the triplet state correction, and the diffusion term (Methods). The two lifetimes $(\tau=1 / \mathrm{k})$ as estimated by fitting, one in the tens of $\mu$ s and the other in the $\mu$ s time range, were observed in all tested complexes, regardless of the presence of the W6 residue that causes intra-chain PET (Table 2). However, the $\tau_{1}$ and $\tau_{2}$ values are different for the respective W6 and W6F constructs, indicating that part of the effect is due to intra-chain PET. Taking into account the known timescales of peptide dynamics in solution (Luitz et al., 2017; Lum et al., 2012a; Neuweiler et al., 2010b), the shorter relaxation time $\tau_{1}$ most likely reflects the quenching interactions of the fluorophore with the internal Trp or the quencher at the ribosome surface. The slower relaxation time $\tau_{2}$ is usually attributed to the dynamics of conformational intermediates. To challenge this assignment, we designed two additional RNCs where we changed the dynamics of peptide chain rearrangements. Because long and unstructured loops are known to enhance conformational fluctuations through increased entropy of the folded state (Dagan et al., 2013), we extended a loop between H3 and H4 by five additional Gly residues and generated two RNCs with different lengths of this construct, called 102 loop and 112 loop, respectively. Both $\tau_{1}$ and $\tau_{2}$ values are affected by the loop mutations (Table 2 ). 
Table 2 Results of analytical fits of PET-FCS ACF for RNCs

Each ACF was an average of $\mathrm{N} \geq 8$. All errors are calculated as standard error of the mean and are indicated in the table. Rates $\left(k_{x}\right)$ are in $\mathrm{s}^{-1} . \tau_{1}, \tau_{2}, \tau_{f}$ and $\tau_{d}$ are relaxation time constants of the respective exponents, in $\mathrm{s}, \tau=1 / \mathrm{k}$.

\begin{tabular}{|c|c|c|c|c|c|c|c|c|c|c|c|c|}
\hline Construct & $\mathrm{C}_{1}$ & $\begin{array}{c}\mathrm{k}_{1} \\
\times 10^{6}\end{array}$ & $\begin{array}{c}\tau_{1} \\
\times 10^{-7}\end{array}$ & $\mathrm{C}_{2}$ & $\begin{array}{c}\mathrm{k}_{2} \\
\mathrm{x} 10^{5}\end{array}$ & $\begin{array}{c}\tau_{2} \\
\times 10 \\
6\end{array}$ & $\mathrm{~F}$ & $\begin{array}{c}\mathrm{k}_{\mathrm{f}} \\
\times 10^{4}\end{array}$ & $\begin{array}{c}\tau_{f} \\
x 10^{-5}\end{array}$ & $\mathrm{~N}$ & $\begin{array}{c}\mathrm{k}_{\mathrm{d}} \\
\mathrm{x} 10^{3}\end{array}$ & $\tau_{d}$ \\
\hline 70 W6 & $\begin{array}{c}0.17 \\
\pm \\
0.01\end{array}$ & $\begin{array}{c}2.58 \\
\pm \\
0.21\end{array}$ & 3.87 & $\begin{array}{c}0.68 \\
\pm \\
0.01\end{array}$ & $\begin{array}{c}4.55 \\
\pm \\
0.09\end{array}$ & 2.20 & $\begin{array}{c}0.18 \\
\pm \\
0.003\end{array}$ & $\begin{array}{c}2.29 \\
\pm \\
0.09\end{array}$ & 4.38 & $\begin{array}{c}0.93 \\
\pm \\
0.003\end{array}$ & $\begin{array}{c}1.05 \\
\pm \\
0.008\end{array}$ & 0.001 \\
\hline $70 \mathrm{~W} 6 \mathrm{~F}$ & $\begin{array}{c}0.14 \\
\pm \\
0.01\end{array}$ & $\begin{array}{c}2.29 \\
\pm \\
0.19\end{array}$ & 4.36 & $\begin{array}{c}0.62 \\
\pm \\
0.01\end{array}$ & $\begin{array}{c}4.59 \\
\pm \\
0.09\end{array}$ & 2.18 & $\begin{array}{c}0.13 \\
\pm \\
0.002\end{array}$ & $\begin{array}{c}2.45 \\
\pm \\
0.10\end{array}$ & 4.09 & $\begin{array}{c}0.93 \\
\pm \\
0.002\end{array}$ & $\begin{array}{c}0.95 \\
\pm \\
0.005\end{array}$ & 0.001 \\
\hline 70 4xA W6 & $\begin{array}{c}0.18 \\
\pm \\
0.01\end{array}$ & $\begin{array}{c}2.67 \\
\pm \\
0.16\end{array}$ & 3.74 & $\begin{array}{c}0.69 \\
\pm \\
0.01\end{array}$ & $\begin{array}{c}4.51 \\
\pm \\
0.07\end{array}$ & 2.22 & $\begin{array}{c}0.16 \\
\pm \\
0.002\end{array}$ & $\begin{array}{c}2.45 \\
\pm \\
0.09\end{array}$ & 4.08 & $\begin{array}{c}0.93 \\
\pm \\
0.002\end{array}$ & $\begin{array}{c}0.99 \\
\pm \\
0.006\end{array}$ & 0.001 \\
\hline 70 4xA W6F & $\begin{array}{c}0.13 \\
\pm \\
0.01\end{array}$ & $\begin{array}{c}2.61 \\
\pm \\
0.23\end{array}$ & 3.84 & $\begin{array}{c}0.61 \\
\pm \\
0.01\end{array}$ & $\begin{array}{c}4.68 \\
\pm \\
0.09\end{array}$ & 2.13 & $\begin{array}{c}0.12 \\
\pm \\
0.002\end{array}$ & $\begin{array}{c}2.86 \\
\pm \\
0.14\end{array}$ & 3.49 & $\begin{array}{c}0.92 \\
\pm \\
0.002\end{array}$ & $\begin{array}{c}0.93 \\
\pm \\
0.005\end{array}$ & 0.001 \\
\hline 102 W6 & $\begin{array}{c}0.20 \\
\pm \\
0.01\end{array}$ & $\begin{array}{c}3.30 \\
\pm \\
0.19\end{array}$ & 3.03 & $\begin{array}{c}0.61 \\
\pm \\
0.01\end{array}$ & $\begin{array}{c}4.29 \\
\pm \\
0.07\end{array}$ & 2.33 & $\begin{array}{c}0.18 \\
\pm \\
0.002\end{array}$ & $\begin{array}{c}2.36 \\
\pm \\
0.09\end{array}$ & 4.23 & $\begin{array}{c}0.94 \\
\pm \\
0.002\end{array}$ & $\begin{array}{c}0.95 \\
\pm \\
0.007\end{array}$ & 0.001 \\
\hline 102 W6F & $\begin{array}{c}0.14 \\
\pm \\
0.01\end{array}$ & $\begin{array}{c}2.91 \\
\pm \\
0.23\end{array}$ & 3.43 & $\begin{array}{c}0.56 \\
\pm \\
0.01\end{array}$ & $\begin{array}{c}4.66 \\
\pm \\
0.09\end{array}$ & 2.15 & $\begin{array}{c}0.13 \\
\pm \\
0.002\end{array}$ & $\begin{array}{c}2.54 \\
\pm \\
0.11\end{array}$ & 3.94 & $\begin{array}{c}0.93 \\
\pm \\
0.002\end{array}$ & $\begin{array}{c}0.93 \\
\pm \\
0.005\end{array}$ & 0.001 \\
\hline 102 loop & $\begin{array}{c}0.13 \\
\pm \\
0.01\end{array}$ & $\begin{array}{c}2.89 \\
\pm \\
0.24\end{array}$ & 3.46 & $\begin{array}{c}0.57 \\
\pm \\
0.01\end{array}$ & $\begin{array}{c}4.33 \\
\pm \\
0.08\end{array}$ & 2.31 & $\begin{array}{c}0.14 \\
\pm \\
0.002\end{array}$ & $\begin{array}{c}2.54 \\
\pm \\
0.12\end{array}$ & 3.93 & $\begin{array}{c}0.93 \\
\pm \\
0.002\end{array}$ & $\begin{array}{c}0.86 \\
\pm \\
0.005\end{array}$ & 0.001 \\
\hline 112 loop & $\begin{array}{c}0.14 \\
\pm \\
0.01\end{array}$ & $\begin{array}{c}2.72 \\
\pm \\
0.21\end{array}$ & 3.68 & $\begin{array}{c}0.54 \\
\pm \\
0.01\end{array}$ & $\begin{array}{c}4.24 \\
\pm \\
0.08\end{array}$ & 2.36 & $\begin{array}{c}0.13 \\
\pm \\
0.002\end{array}$ & $\begin{array}{c}2.47 \\
\pm \\
0.11\end{array}$ & 4.05 & $\begin{array}{c}0.93 \\
\pm \\
0.002\end{array}$ & $\begin{array}{c}0.88 \\
\pm \\
0.005\end{array}$ & 0.001 \\
\hline $112 \mathrm{~W} 6$ & $\begin{array}{c}0.19 \\
\pm \\
0.01\end{array}$ & $\begin{array}{c}3.61 \\
\pm \\
0.19\end{array}$ & 2.77 & $\begin{array}{c}0.48 \\
\pm \\
0.01\end{array}$ & $\begin{array}{c}4.41 \\
\pm \\
0.08\end{array}$ & 2.27 & $\begin{array}{c}0.10 \\
\pm \\
0.002\end{array}$ & $\begin{array}{c}2.79 \\
\pm \\
0.16\end{array}$ & 3.59 & $\begin{array}{c}0.93 \\
\pm \\
0.002\end{array}$ & $\begin{array}{c}0.84 \\
\pm \\
0.004\end{array}$ & 0.001 \\
\hline 112 W6F & $\begin{array}{c}0.14 \\
\pm \\
0.01\end{array}$ & $\begin{array}{c}2.94 \\
\pm \\
0.22\end{array}$ & 3.40 & $\begin{array}{c}0.52 \\
\pm \\
0.01\end{array}$ & $\begin{array}{c}4.45 \\
\pm \\
0.09\end{array}$ & 2.25 & $\begin{array}{c}0.11 \\
\pm \\
0.002\end{array}$ & $\begin{array}{c}2.51 \\
\pm \\
0.13\end{array}$ & 3.98 & $\begin{array}{c}0.93 \\
\pm \\
0.002\end{array}$ & $\begin{array}{c}0.91 \\
\pm \\
0.005\end{array}$ & 0.001 \\
\hline 112 4xA W6 & $\begin{array}{c}0.20 \\
\pm \\
0.01\end{array}$ & $\begin{array}{c}2.16 \\
\pm \\
0.13\end{array}$ & 4.63 & $\begin{array}{c}0.59 \\
\pm \\
0.01\end{array}$ & $\begin{array}{c}3.73 \\
\pm \\
0.08\end{array}$ & 2.68 & $\begin{array}{c}0.21 \\
\pm \\
0.003\end{array}$ & $\begin{array}{c}1.76 \\
\pm \\
0.06\end{array}$ & 5.68 & $\begin{array}{c}0.95 \\
\pm \\
0.003\end{array}$ & $\begin{array}{c}1.00 \\
\pm \\
0.008\end{array}$ & 0.001 \\
\hline $1124 \times A$ W6F & $\begin{array}{c}0.12 \\
\pm \\
0.01\end{array}$ & $\begin{array}{c}2.52 \\
\pm \\
0.23\end{array}$ & 3.97 & $\begin{array}{c}0.43 \\
\pm \\
0.01\end{array}$ & $\begin{array}{c}3.94 \\
\pm \\
0.97\end{array}$ & 2.54 & $\begin{array}{c}0.11 \\
\pm \\
0.002\end{array}$ & $\begin{array}{c}2.30 \\
\pm \\
0.13\end{array}$ & 4.35 & $\begin{array}{c}0.93 \\
\pm \\
0.002\end{array}$ & $\begin{array}{c}0.86 \\
\pm \\
0.005\end{array}$ & 0.001 \\
\hline
\end{tabular}


Because $\tau_{1}$ and $\tau_{2}$ are apparent values that have no biological meaning as such, in the following we develop a kinetic modeling approach to determine the elemental rates of nascent chain dynamics. To be able to use commercial software for global fitting, we extract the dynamic component from the raw ACF curves by subtracting from each experimental ACF the diffusional and triplet state components using parameters of the analytical fit (Equation 1; Table 2). This results in time courses with two exponential decays (Figure 16b).

\section{Kinetic model for the dynamics of nascent chains.}

To fit the ACF data, we built several kinetic models to determine the minimal model that could best fit the recorded data. First, we tried the simplest two equilibria model - Model 1. This presents three possible states of the nascent chain: State $\mathrm{C}$ - nascent chain on the ribosome, with unquenched ATTO655 dye; R - nascent chain interacting with the ribosome and the dye quenched by guanines of the ribosome; $\mathrm{W}$ - dye quenched by the intrachain tryptophan Trp6 (Figure 16a). The fitting resulted in poorly defined rates and high residual values (Figure 16b).

Next, we performed a fitting to a three equilibria model - Model 2. Here we introduced an additional equilibrium that could characterize the conformational fluctuations of the nascent chain on the ribosome; however, we maintained that only one of these states could undergo interactions with the ribosome and intrachain tryptophan that would result in fluorescence quenching (Figure 17a). This model was unable to sufficiently fit the data, again resulting in poorly defined rates and comparing the fit to the recorded data we observed high residual values (Figure 17b). 
a<smiles>[R]C1=C([As])[C@@H]1[W]</smiles>

b
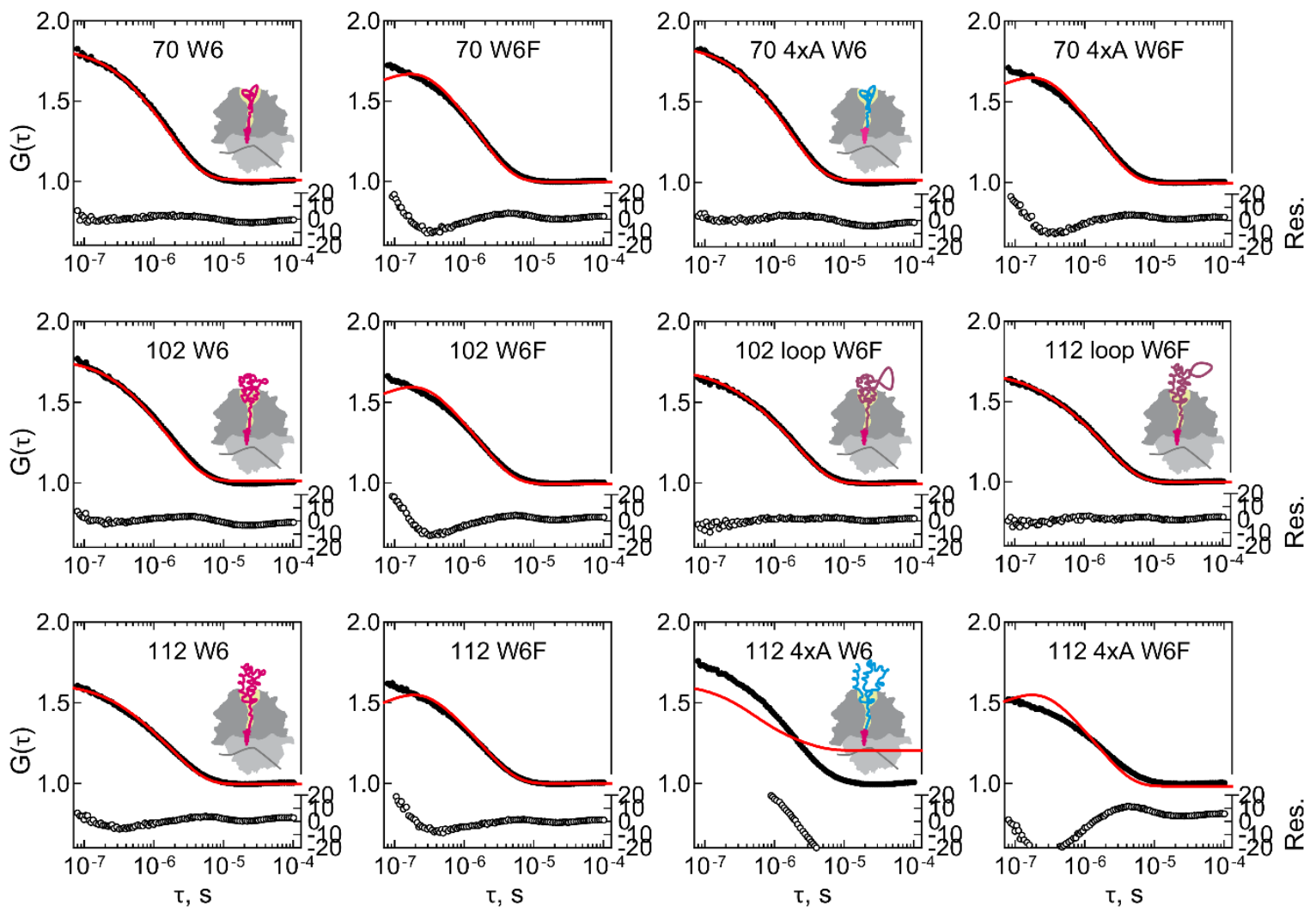

Figure 16 Kinetic model 1 of conformational dynamics of HemK RNCs

a. The kinetic model: state $\mathrm{C}$ is the nascent chain on the ribosome, $\mathrm{W}-\operatorname{Trp}$-quenched state; $\mathrm{R}-$ ribosome-quenched state.

b. Results of global fitting of autocorrelation data to the kinetic model. Black - measured data, red - kinetic model simulation curve, both plotted on the left-hand y axis; open circles - residual values plotted on right-hand y axis (Res.); Cartoons indicate the presumed position of the nascent chains on the ribosome, with the wt nascent chains shown in magenta, without and with an extra loop, and the 4xA variant shown in blue. 
a

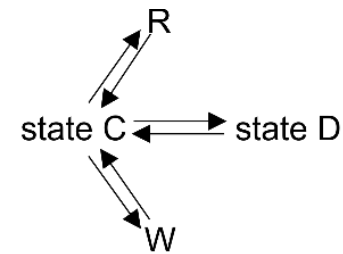

b
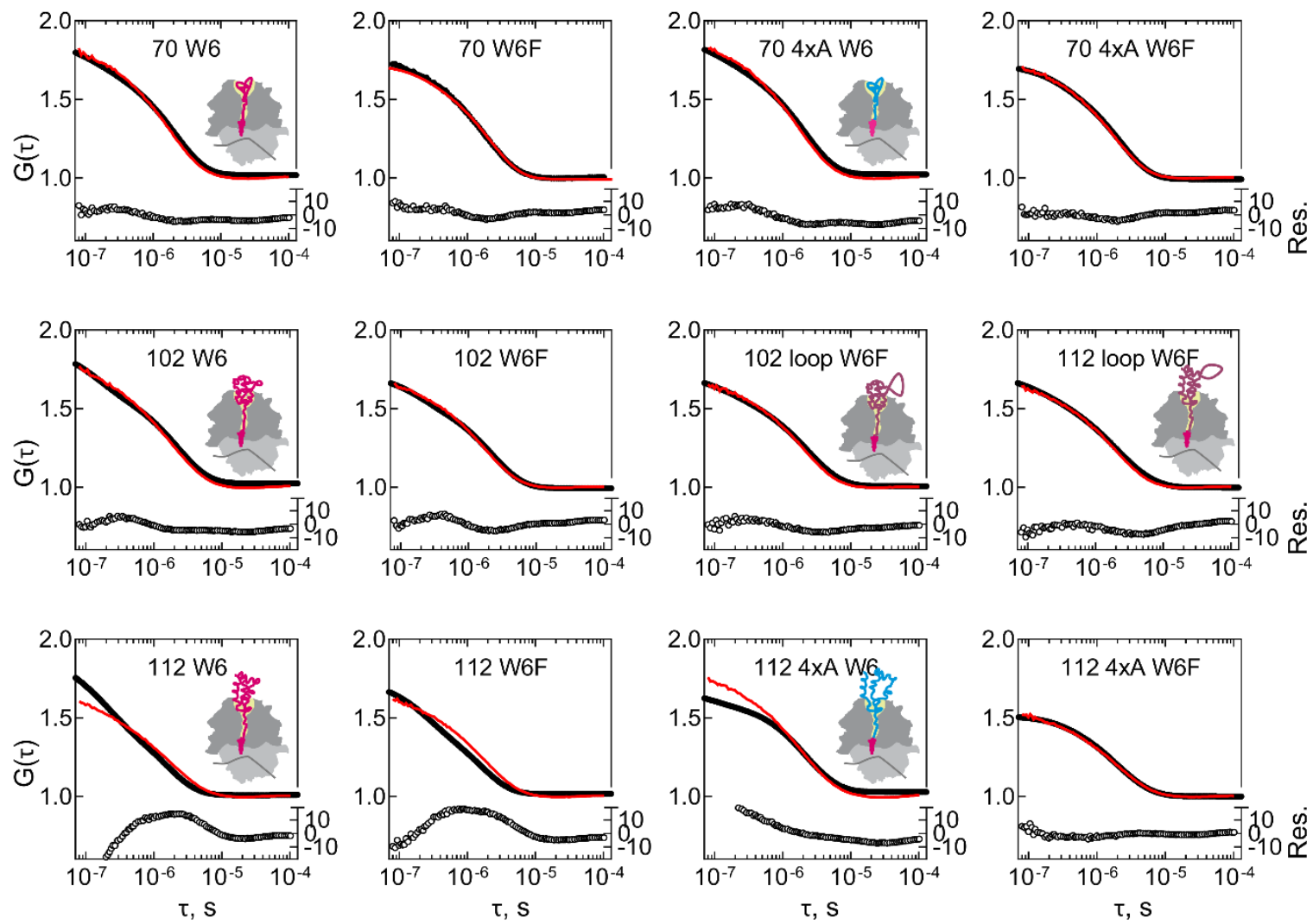

Figure 17 Kinetic model 2 of conformational dynamics of HemK RNCs

a. The kinetic model: State $C$ and $D$ are the nascent chain conformations on the ribosome, $W$ Trp-quenched state; $\mathrm{R}$ - ribosome-quenched state.

b. Results of global fitting of autocorrelation data to the kinetic model (Labeling as in Figure 16).

In kinetic model 3 to account for the exponential term on the $\mu$ s time scale, we assume that nascent chains can undergo a conformational change from state $C$ to state $D(C \leftrightarrow D)$ (Figure 18a), and that in each of these states the N-terminal dye can interact with the internal Trp6. The resulting quenched state is denoted as $W$, leading to the equilibria $C \leftrightarrow W$ and $D \leftrightarrow W$. By analogy, a quencher on the ribosome surface can quench the fluorescence of $C$ or $D$, yielding a non-fluorescent state $R$ and the equilibria $C \leftrightarrow R$ and $D \leftrightarrow R$ (Figure 18a). This is the minimal model that could reliably fit the ACF data (Figure 18b). 
a

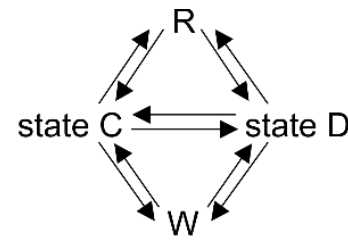

b
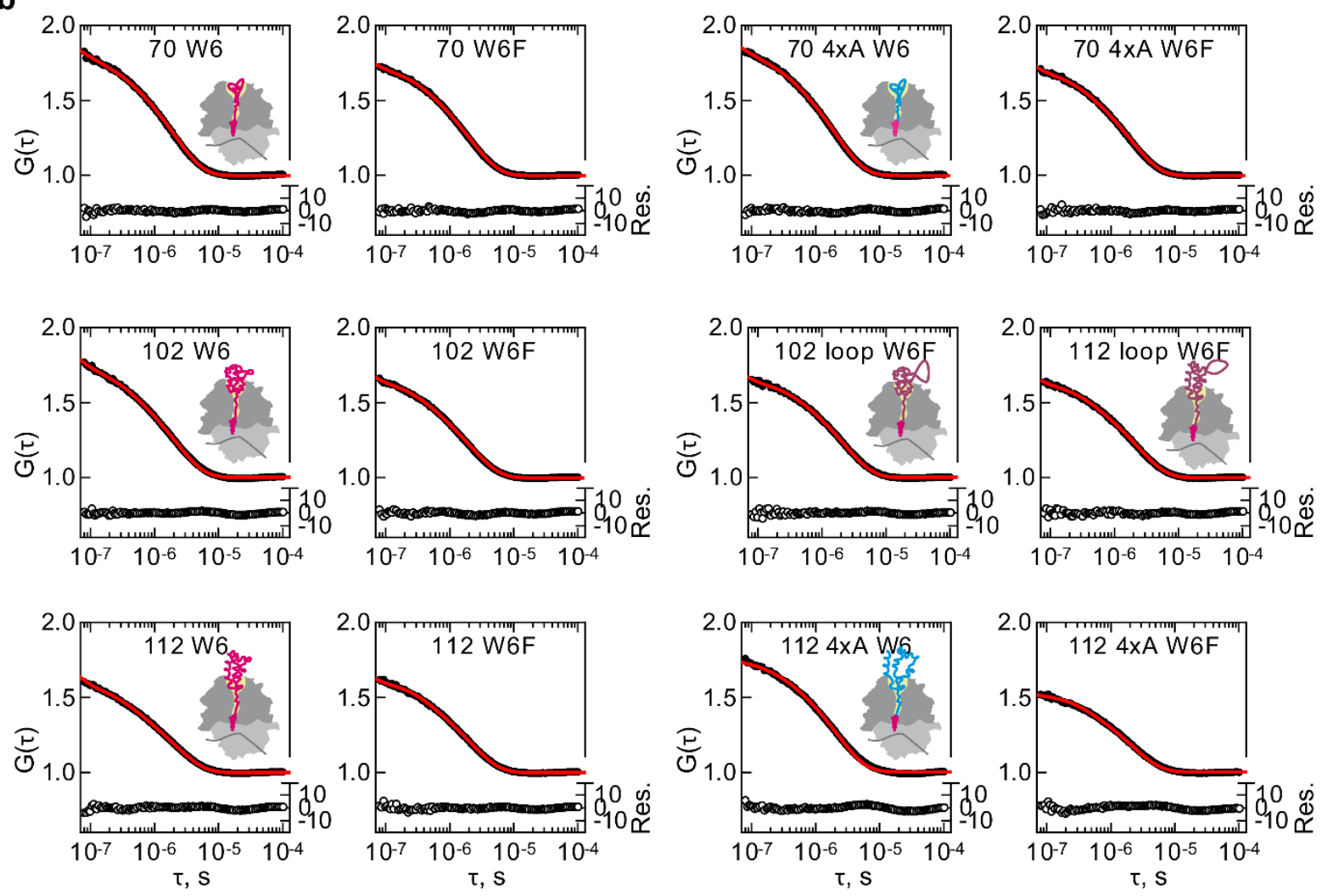

Figure 18 Kinetic model 3 of conformational dynamics of HemK RNCs

Shown are results of global fitting of autocorrelation data to the kinetic model (panel a) C and D are two peptide conformations; $W$-Trp-quenched state; $R$ - ribosome-quenched state. $b$ - Black measured data; red - kinetic model simulation curve, open circles - residuals. Cartoons indicate the presumed position of the nascent chains on the ribosome (color-coding same as Figure 16).

We perform a global fit of the entire dataset in order to determine the elemental rates of the outlined interactions. Because there is no solution to calculate elemental rates from the two apparent rate constants, we additionally measured ACF of RNC 70 W6F and RNC 102 W6F at different free Trp concentrations (Figure 19, Table 3). 
a

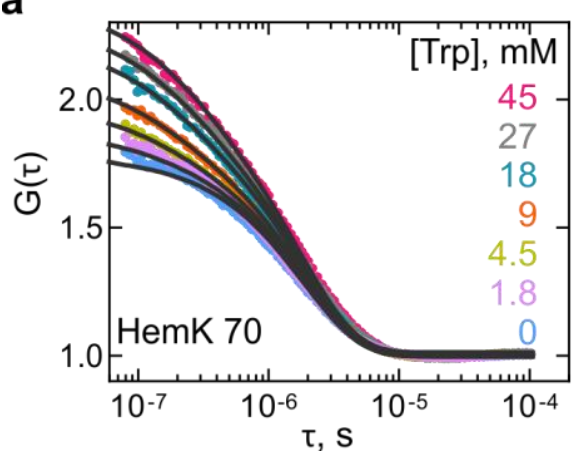

b

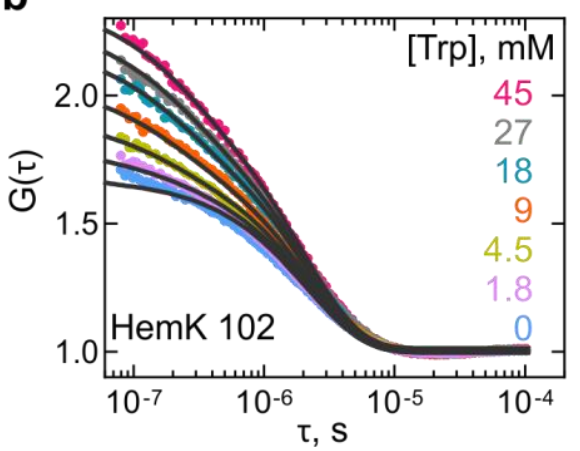

C

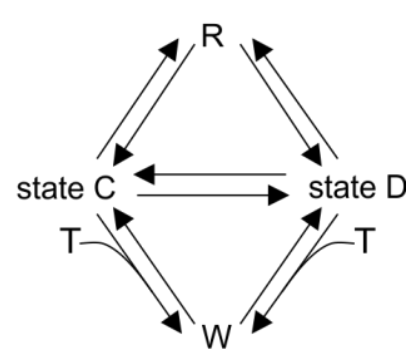

Figure 19 Global fitting of HemK W6F RNC ACFs with increasing free Trp concentration

Free Trp concentration is indicated on the right of each graph.

a. HemK 70 W6F RNC

b. HemK 102 W6F RNC

c. Global fit kinetic model. R - Ribosome quenched state, W - tryptophan quenched state,

State $C$ and state $D$ - conformational states of the nascent peptide, $T$ - free tryptophan.

\section{Table 3 Results of global fitting of the free Trp titration dataset}

Rates that were linked during global fitting are shown in the same cell shade. Rates are in $\mu^{-1}$, except the $W \mathrm{k}_{\text {on }}$ rates that are in $\mathrm{mM}^{-1} \mu \mathrm{s}^{-1}$. A covariance matrix derived using nonlinear regression algorithms is used to estimate the standard errors by the Kintek Explorer software.

\begin{tabular}{|c|c|c|c|c|c|c|}
\hline Construct & $k$ & $\mathrm{D} \leftrightarrow \mathrm{R}$ & $\mathrm{C} \leftrightarrow \mathrm{R}$ & $\mathrm{D} \leftrightarrow \mathrm{W}$ & $\mathrm{C} \leftrightarrow \mathrm{W}$ & $\mathrm{D} \leftrightarrow \mathrm{C}$ \\
\hline \multirow{2}{*}{$70 \mathrm{~W} 6 \mathrm{~F}$} & on & $83 \pm 17$ & $0.5 \pm 0.06$ & $0.006 \pm 0.003$ & $(8.0 \pm 0.5) \times 10^{-7}$ & $9.6 \pm 12.1$ \\
\cline { 2 - 7 } & off & $0.0002 \pm 0.002$ & $0.01 \pm 0.06$ & $2 \pm 0.1$ & $2 \pm 0.1$ & $0.001 \pm 0.1$ \\
\hline \multirow{2}{*}{$102 \mathrm{~W} 6 \mathrm{~F}$} & on & $85 \pm 15$ & $0.5 \pm 0.05$ & $0.007 \pm 0.003$ & $(8.0 \pm 0.5) \times 10^{-7}$ & $3 \pm 10.4$ \\
\cline { 2 - 7 } & off & $0.0002 \pm 0.002$ & $0.01 \pm 0.06$ & $2 \pm 0.1$ & $2 \pm 0.1$ & $0.0004 \pm 0.1$ \\
\hline
\end{tabular}

Global fit of the Trp titration data yielded the ATTP655-Trp dequenching rate (Methods), $2.0 \pm 0.1 \mu^{-1}$ (Table 3), which was fixed during global fitting of the main dataset (Figure 18, Figure 20, Table 4) and allowed us to obtain statistically significant values for rate constants describing nascent chain dynamics of each HemK NTD variant on the ribosome (Figure 20, Table 4 and Table 8 in Methods). The only parameters that were poorly defined are those where the rate constants were extremely low and therefore significantly slower than the RNC diffusion through the confocal volume. This is expected, as reliable FCS measurements become increasingly difficult when the relaxation times exceed $1 \mathrm{~s}$ (Elson, 2018; Meseth et al., 1999). 

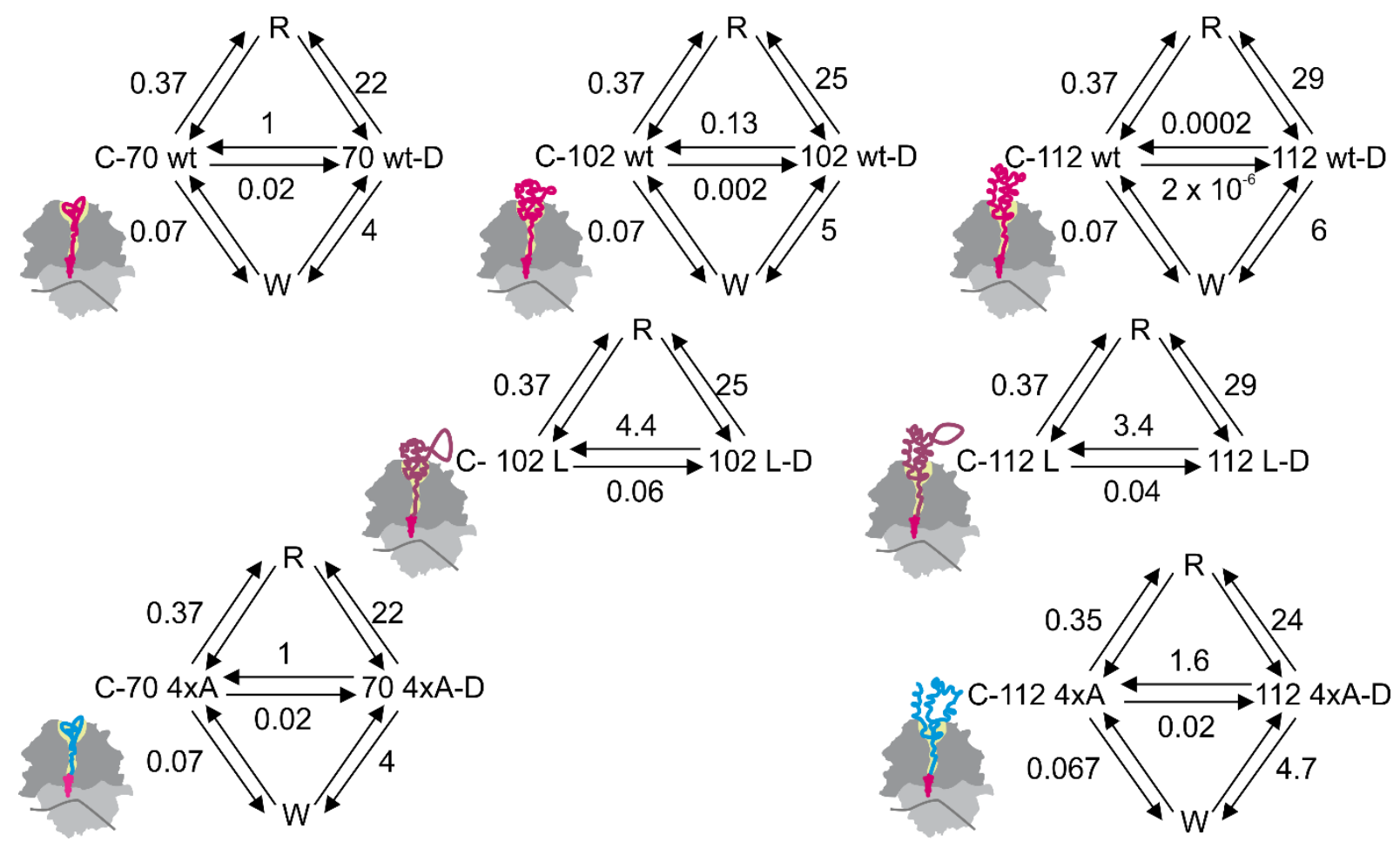

Figure 20 Rates of the conformational dynamics of HemK RNCs

All color-coding and cartoons consistent with figure 16. Errors of rates displayed in Table 4.

\section{Table 4 Results of global fitting of HemK RNCs}

Rates that were linked during global fit are shown in the same cell shade; locked rates are in red. Rates are in $\mu \mathrm{s}^{-1}$. A covariance matrix derived using nonlinear regression algorithms is used to estimate the standard errors by the Kintek Explorer software.

\begin{tabular}{|l|c|c|c|c|c|c|}
\hline Construct & $\mathrm{k}$ & $\mathrm{D} \leftrightarrow \mathrm{R}$ & $\mathrm{C} \leftrightarrow \mathrm{R}$ & $\mathrm{D} \leftrightarrow \mathrm{W}$ & $\mathrm{C} \leftrightarrow \mathrm{W}$ & $\mathrm{D} \leftrightarrow \mathrm{C}$ \\
\hline $70 \mathrm{wt}$ & on & $22 \pm 3.3$ & $0.37 \pm 0.02$ & $4.2 \pm 3$ & $0.07 \pm 0.03$ & $0.975 \pm 0.3$ \\
\cline { 2 - 7 } & off & $0.3 \pm 0.02$ & $0.3 \pm 0.02$ & 2 & 2 & $0.0167 \pm 0.02$ \\
\hline $704 \times \mathrm{A}$ & on & $22 \pm 3.3$ & $0.37 \pm 0.02$ & $4.2 \pm 3$ & $0.07 \pm 0.03$ & $0.975 \pm 0.3$ \\
\cline { 2 - 7 } & off & $0.3 \pm 0.02$ & $0.3 \pm 0.02$ & 2 & 2 & $0.0167 \pm 0.02$ \\
\hline \multirow{3}{*}{$102 \mathrm{wt}$} & on & $25 \pm 1.2$ & $0.37 \pm 0.02$ & $4.8 \pm 1$ & $0.07 \pm 0.03$ & $0.13 \pm 0.3$ \\
\cline { 2 - 7 } & off & $0.3 \pm 0.02$ & $0.3 \pm 0.02$ & 2 & 2 & $0.002 \pm 0.02$ \\
\hline 102 loop & on & $25 \pm 1.2$ & $0.37 \pm 0.02$ & & & $4.39 \pm 0.5$ \\
\cline { 2 - 7 } & off & $0.3 \pm 0.02$ & $0.3 \pm 0.02$ & & & $0.0653 \pm 0.03$ \\
\hline 112 loop & on & $29 \pm 1.3$ & $0.37 \pm 0.02$ & & & $3.44 \pm 0.5$ \\
\cline { 2 - 7 } & off & $0.3 \pm 0.02$ & $0.3 \pm 0.02$ & & & $0.0442 \pm 0.03$ \\
\hline $112 \mathrm{wt}$ & on & $29 \pm 1.3$ & $0.37 \pm 0.02$ & $5.6 \pm 1$ & $0.07 \pm 0.03$ & $0.0002 \pm 0.3$ \\
\cline { 2 - 7 } & off & $0.3 \pm 0.02$ & $0.3 \pm 0.02$ & 2 & 2 & $0.000002 \pm 0.02$ \\
\hline $1124 \times A$ & on & $24 \pm 8.2$ & $0.35 \pm 0.02$ & $4.7 \pm 8$ & $0.07 \pm 0.01$ & $1.62 \pm 0.4$ \\
\cline { 2 - 7 } & off & $0.3 \pm 0.02$ & $0.3 \pm 0.02$ & 2 & 2 & $0.0232 \pm 0.1$ \\
\hline
\end{tabular}


Comparison of the rate constants for different complexes reveals, first, that the two structural states of the nascent chain, $C$ and $D$, are very different with respect to their dynamics (Figure 20 , Table 4). The rate constants of ATTO655 quenching by the ribosome is $60-80$-fold slower in $C$ compared to $D$ in different RNCs. Likewise, quenching by intramolecular Trp is 60-100fold slower in $\mathrm{C}$ than in $\mathrm{D}$. We attribute this to lower dynamics of state $\mathrm{C}$ and thus denote it as compact, compared to state $\mathrm{D}$, which we call dynamic. Thus, we identify two global conformational ensembles of ribosome-bound HemK NTD.

The second observation is that for a given conformational state $C$ or $D$, the rates of interaction with Trp or the ribosome are almost insensitive to the extent of folding or the position of the $\mathrm{N}$ terminus on the ribosome, as the $\mathrm{k}_{\mathrm{on}}$ value for Trp quenching is about $0.07 \mu \mathrm{s}^{-1}$ for state $\mathrm{C}$ and 4-6 $\mu \mathrm{s}^{-1}$ for state $\mathrm{D}$ of all constructs (Figure 20). The low quenching rate constant in the compact states suggests that Trp is confined in the stable hydrophobic core of the respective intermediates. In contrast, the dynamic state presents a less compact structure wherein the more exposed native Trp interacts more frequently with the $\mathrm{N}$-terminal dye. It is possible that the position of the $\mathrm{N}$-terminus is defined at early stages of folding, which explains why it does not change with the peptide length over 70 aa. More surprisingly, the $k_{\text {on }}$ for ribosome quenching is not strongly dependent on the position of the nascent chain with respect to the ribosome, with $\mathrm{k}_{\mathrm{on}}=0.37 \mu \mathrm{s}^{-1}$ for state $\mathrm{C}$ and ranging from $22 \mu \mathrm{s}^{-1}$ to $29 \mu \mathrm{s}^{-1}$ for state $\mathrm{D}$. The dequenching rate of the ATTO655-ribosome complex is $0.3 \mu \mathrm{s}^{-1}$.

The largest difference related to the stage of nascent peptide folding and its position on the ribosome pertains to the equilibrium between states $C$ and $D$. The compact state $C$ is favored in all complexes, but the rate constants of the $C \leftrightarrow D$ transitions differ dramatically, both decreasing with increasing protein length and its emergence outside the ribosome (Figure 20). Disruption of the hydrophobic core by 4xA mutations does not alter the equilibrium between the $C$ and $D$ states in HemK70, but strongly destabilizes the NTD when it emerges from the ribosome (HemK112). Furthermore, extension of a loop by 6 aa dramatically increases the rates of transitions in both HemK102 and HemK112 (Table 4). Thus, as predicted (Dagan et al., 2013), the loop extensions increase the helix dynamics of the nascent chain, but do not alter the interaction with Trp or the ribosome surface.

Comparison of the free energy of the transition state $\left(\Delta \mathrm{G}^{\ddagger}\right)$ barrier between the compact and dynamic states at different nascent chain lengths shows that as the nascent chain grows, transition state free energy increases from $38 \mathrm{~kJ} \mathrm{~mol}^{-1}$ for HemK70 to $43 \mathrm{~kJ} \mathrm{~mol}^{-1}$ for HemK102 and to $59 \mathrm{~kJ} \mathrm{~mol}^{-1}$ for HemK112 (Figure 21b, Table 5). 

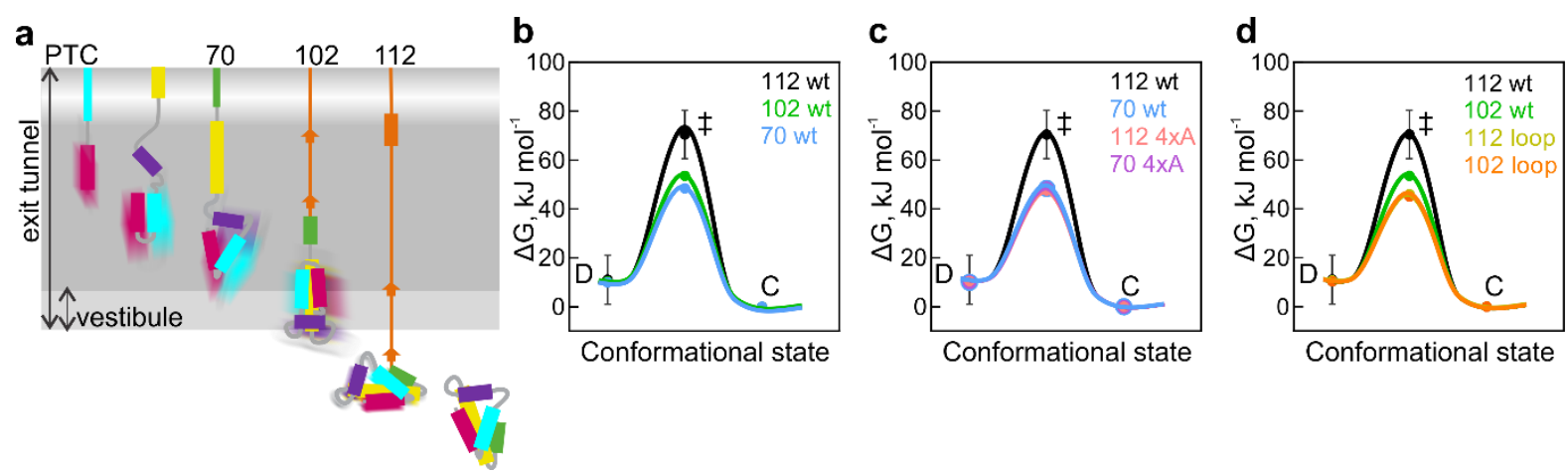

\section{Figure 21 Free energy barriers between different chain conformations}

D-dynamic state; C - compact state; $\ddagger$ - transition state. Error bars represent propagated errors from elemental rates derived from the kinetic fits.

a. Schematic diagram of cotranslational folding steps of HemK NTD with a visualization of nascent chain dynamics.

b. HemK wt constructs of increasing length, 70 aa (blue), 102 (green), and 112 (black).

c. HemK wt and 4xA variants, 70 wt (blue) 70 4xA (lilac), 112 (black), and 112 4xA (red).

d. HemK wt and loop variants, 102 wt (green), 112 wt (black), 102 loop (orange), and 112 loop (yellow).

\section{Table 5 Free energy calculations for HemK RNC constructs}

Elemental rate errors from the kinetic fits are propagated through Equation 2 (Methods).

\begin{tabular}{|l|l|l|l|}
\hline Construct & $\Delta \mathrm{G}^{\circ} \mathrm{D}-\Delta \mathrm{G}^{\ddagger}, \mathrm{kJ} \mathrm{mol}^{-1}$ & $\Delta \mathrm{G}^{\circ}{ }_{\mathrm{C}}-\Delta \mathrm{G}^{\ddagger}, \mathrm{kJ} \mathrm{mol}^{-1}$ & $\Delta \mathrm{G}^{\circ} \mathrm{D}-\Delta \mathrm{G}^{\circ}{ }_{\mathrm{C}}, \mathrm{kJ} \mathrm{mol}^{-1}$ \\
\hline $70 \mathrm{wt}$ & $38.4 \pm 0.0003$ & $48.4 \pm 0.001$ & $10.0 \pm 0.001$ \\
\hline $704 \times \mathrm{A}$ & $38.4 \pm 0.0003$ & $48.4 \pm 0.0000$ & $10.0 \pm 0.0003$ \\
\hline $102 \mathrm{wt}$ & $43.3 \pm 0.002$ & $53.6 \pm 0.01$ & $10.3 \pm 0.01$ \\
\hline 102 loop & $34.7 \pm 0.0001$ & $45.0 \pm 0.0005$ & $10.3 \pm 0.0005$ \\
\hline 112 loop & $35.3 \pm 0.0001$ & $46.0 \pm 0.0007$ & $10.7 \pm 0.0007$ \\
\hline $112 \mathrm{wt}$ & $59.2 \pm 1.5$ & $70.5 \pm 10$ & $11.3 \pm 10.1$ \\
\hline $1124 \times A$ & $37.2 \pm 0.0002$ & $47.6 \pm 0.004$ & $10.4 \pm 0.004$ \\
\hline
\end{tabular}




\section{SECTION 4 - Discussion}

In this work, we combined FPA and PET-FCS to reconstruct the trajectory of cotranslational folding and to evaluate the stability of the folding intermediates of HemK NTD. The highresolution FPA data show that nascent chains undergo sequential force-generating rearrangements that start inside the exit tunnel as soon as individual $\alpha$-helices pass the constriction site. The earliest detected force-generating folding intermediate entails as little as a single helix $(\mathrm{H} 1)$ of the HemK NTD. Upon continued synthesis, emerging helices begin to interact with one another forming tertiary intermediates inside the peptide exit tunnel (Figure 10d). Our results combined with previous data (Kemp et al., 2019; Mercier and Rodnina, 2018) show that folding of HemK NTD is sequential and that there are several tension-generating steps corresponding to folding intermediates inside and outside of the ribosome. The formation of individual $\alpha$-helices and tertiary interactions between $\alpha$-helical elements within the exit tunnel are well documented (Bhushan et al., 2010; Farias-Rico et al., 2018; Lu and Deutsch, 2005; Nilsson et al., 2015; Nissley and O'Brien, 2018). FPA studies also demonstrate that the growing nascent chain continues to undergo structural adjustments after emerging from the exit tunnel; some, but not all, of these rearrangements are sensitive to the packing of the protein's hydrophobic core. In contrast, folding of the HemK NTD in solution is concerted, with only two discernible states, native and unfolded (Holtkamp et al., 2015). Thus, translation rate and sequential addition of amino acids during translation affects nascent-protein folding not only inside the exit tunnel, but also at the surface of the ribosome and results in a complex folding pathway with multiple folding intermediates. Cotranslational folding is under combined thermodynamic and kinetic control. The kinetics of translation can change the states that the nascent chain can access on the ribosome, while different conditions in the surrounding solvent or effects from the ribosome itself as well as the properties of amino acids in a nascent chain segment determine the free energy landscape for the particular conformation (Baker and Agard, 1994; Varela et al., 2019). There is an implicit assumption in folding studies that the final native state is the most thermodynamically stable state, however this might be true only in the context of kinetically accessible states (Baker and Agard, 1994). The purpose of kinetically optimized sequential cotranslational folding may be to ensure that only the productive on-pathway states are accessible to the nascent chain.

Rapid sequential cotranslational folding observed here for HemK NTD can be rationalized using the concept of folding via cooperative folding units, foldons, which was suggested for several proteins based on a combination of NMR, mass spectrometry, and hydrogen-exchange pulse-labeling experiments (Bai et al., 1995; Hu et al., 2013; Walters et al., 2013). The emerging view is that the foldons, comprised of one to two secondary structure elements, form rapidly (e.g. at a rate of $2000 \mathrm{~s}^{-1}$ in $\mathrm{RNaseH}$ (Hu et al., 2013)), and once a foldon is formed, 
the protein undergoes a series of fast folding steps, with native-like foldons added rapidly at each step. The trajectory of how a particular protein folds is determined by the nature of foldon units, because each preceding unit guides and stabilizes the incoming foldons in a thermodynamically downhill energy landscape (Englander and Mayne, 2014). The vectorial emergence of nascent peptide into the constrained space of the exit tunnel may allow nucleation of such folding units and restrict the number of potential interactions/conformations at a given chain length, thereby guiding folding through a relatively narrow energy landscape. This would also explain why folding of the HemK NTD on the ribosome is sequential, guided by the vectorial appearance of foldons during translation. In solution, folding is concerted because formation of local folding units defines the rate of the concerted collapse into the native structure.

The PET-FCS experiments show that nascent peptides are dynamic and undergo internal conformational rearrangements on a $\mu$ s time scale. We identify two subpopulations of folded nascent chains corresponding to the predominant compact and dynamic states, which differ in their ability to interact with the local environment. The local dynamics of the N-terminus as monitored by intramolecular PET between the N-terminal ATTO655 and Trp6 is relatively slow for the compact state $\left(0.07 \mu \mathrm{s}^{-1}\right)$. The dynamic state has quenching $\left(4-6 \mu \mathrm{s}^{-1}\right)$ and dequenching $\left(2 \mu \mathrm{s}^{-1}\right)$ rates similar to those reported for the dynamic motion of proteins (Luitz et al., 2017; Lum et al., 2012a; Neuweiler et al., 2010b; Neuweiler et al., 2009; Stanley et al., 2014). The quenching interaction with the ribosome is also slow for the compact (0.3-0.4 $\left.\mu \mathrm{s}^{-1}\right)$ and fast for the dynamic $\left(20-30 \mu \mathrm{s}^{-1}\right)$ state; the dequenching rate is $0.3 \mu \mathrm{s}^{-1}$. The latter rates differ from the dequenching of the ATTO655-Trp complex and most probably reflect the interactions of ATTO655 with guanine residues in rRNA. This is the first time these interactions have been characterized in a context of an RNA-containing macromolecule.

During folding in solution, the rates of fluctuations slow down several-fold as proteins advance from unfolded towards more compact conformations (Nettels et al., 2007; Waldauer et al., 2010). On the ribosome, the rates of fluctuations between the compact and dynamic states decrease as the nascent chain moves down the exit tunnel and emerges from the ribosome (Figure 21a). The large increase in $\Delta G^{\ddagger}$ as the NTD moves away from the ribosome demonstrates how the proximity of the ribosome alters the dynamics of the nascent protein domain. Consistently with these results, isolated HemK NTD did not show any PET dynamics. Although the protein can unfold in solution (at a rate of $1 \mathrm{~s}^{-1}$ at $37^{\circ} \mathrm{C}$ ), such dynamics is probably too slow for the FCS and the unfolded state is too rare to be detected due to rapid folding (2000 $\mathrm{s}^{-1}$ ) (Holtkamp et al., 2015). The rates of the free HemK NTD protein folding in solution (Holtkamp et al., 2015) are within the range of rates of conformational fluctuations $C \leftrightarrow D$ of HemK112 on the ribosome, suggesting that the dynamic conformation of HemK112 is similar 
to the unfolded state in solution. These data provide further support to the notion that the stability of protein domains increases with the distance to the ribosome (Alexander et al., 2019; Cabrita et al., 2016; Kaiser et al., 2011; Liu et al., 2017; Samelson et al., 2016) and provide estimations for the rates of rapid conformational fluctuations of nascent proteins at different stages of folding.

The 4xA mutations destabilize the native hydrophobic packing of the domain and have a significant effect on native tertiary interactions (Holtkamp et al., 2015). The FPA results show that nascent wt and 4XA NTDs form very similar compact intermediates inside the exit tunnel. PET-FCS analysis indicates that the dynamic fluctuations between compact and dynamic states have identical transition state barriers $\left(\Delta \mathrm{G}^{\ddagger}=38.4 \mathrm{~kJ} \mathrm{~mol}^{-1}\right)$ for wt and $4 \times \mathrm{XA} \mathrm{HemK70}$ (Figure $21 \mathrm{c}$ ) and, in both cases, the respective intermediates are highly dynamic. In contrast, outside the tunnel, mutations in the hydrophobic core or loop extensions increase dynamic fluctuations of the nascent chains (Figure 21c,d; Table 5). In particular for the stably folded HemK112, the effect of the mutations is very large, decreasing the $\Delta \mathrm{G}^{\ddagger}$ value by $22-25 \mathrm{~kJ} \mathrm{~mol}^{-1}$ (Figure $21 \mathrm{c}, \mathrm{d}$ ). The free-energy landscapes of protein folding in solution are generally shallow, i.e. the differences between the highs (barriers) and lows (energy minima) are in the tens of $\mathrm{kJ}$ $\mathrm{mol}^{-1}$ rather than hundreds (Gruebele et al., 2016). For example, the differences between partially unfolded high-energy states and the native states of proteins are between 17-54 kJ $\mathrm{mol}^{-1}$ (Englander and Mayne, 2014). In the case of the HemK NTD, the folding free energy of this isolated domain is $20.8 \mathrm{~kJ} \mathrm{~mol}^{-1}$ (Holtkamp et al., 2015). This suggests that the $4 \times A$ and loop extension variants of the NTD on the ribosome are energetically analogous to the unfolded isolated domain in solution.

In summary, the present work shows how a small a-helical protein domain folds cotranslationally. It starts folding as soon as the first helical elements pass the constriction of the exit tunnel of the ribosome. With growing nascent chain, the emerging helical segments dock onto each other sequentially. The folding pathway entails numerous intermediates that continue to rearrange even when the domain emerges from the ribosome. Inside the nascent tunnel or in the proximity of the ribosome the nascent peptide is highly dynamic, undergoing structural fluctuations on the $\mu$ s time scale. The fluctuations slow down as the domain moves away (or is released) from the ribosome. Destabilizing mutations have little effect on folding within the exit tunnel, but abolish the domain stabilization after its separation from the ribosome. The results show the power of FPA and PET-FCS in solving the trajectory of cotranslational protein folding and in characterizing the dynamic properties of folding intermediates. 
One consistent aspect of cotranslational folding seems to be that during translation the cotranslational intermediates formed in the vicinity of the ribosome surface are destabilized. We show this destabilizing effect for an a-helical protein HemK NTD, but it was also previously shown for an $\alpha$-helical T4 lysozyme (Kaiser et al., 2011), and a predominantly $\beta$-sheet proteins GFP, RFP (Kelkar et al., 2012) and FLN5 (Deckert et al., 2016) proteins, as well as a complex $\alpha / \beta$ fold RnaseH (Samelson et al., 2016). The fact that this phenomena has been observed in many cases and on many diverse folds suggests that the destabilization of structures in the vicinity of the ribosome is the rule rather than exception during cotranslational folding.

The nearly perfect vectorial folding as helixes emerge sequentially from the constriction site at this time has only been observed for HemK NTD. Therefore, it is still unclear whether the case of HemK is an exception or indication of a previously overlooked phenomenon. The force profile assay has previously indicated potential folding intermediates (i.e., multiple high-tension peaks) for all $\alpha$-helical proteins villin and $\lambda$-repressor (Marino et al., 2016) as well as an all $\beta$ sheet protein FLN5 (Kemp et al., 2019). In all these cases, the folding was investigated with an aid of a linker sequence, and possible high-tension intermediates formed inside the exit tunnel have not been investigated. It would be very interesting to investigate a large number of different folds to study the prevalence of true vectorial folding for single domain proteins.

There are several studies that have attempted to estimate the evolutionary age of different protein folds (reviewed in (Ma et al., 2008)). It has been suggested that folds where only one of the secondary structures dominates is a relatively recent evolutionary invention, whereas $\alpha / \beta$ containing folds (Choi and Kim, 2006) like the P-loop containing nucleoside triphosphate hydrolases or the TIM $\alpha / \beta$-barrel folds are significantly older (Choi and Kim, 2006; Ma et al., 2008). A systematic investigation of cotranslational folding for different folds that represent potentially different evolutionary ages could aid in the understanding of the timing of sequential folding emergence, unless sequential folding is an imperative of cotranslational folding. It is important to note that these types of studies of protein age may disproportionately favor catalytically active proteins and can be relatively blind to regulatory proteins (Jain et al., 2019). Therefore, in such a systematic study a variety of functional as well as fold classes should be included. Force profile assay would be an excellent method for such an undertaking, as it has capacity for detecting highly dynamic folding intermediates that would be invisible to structural methods like NMR or cryo-EM.

The PET-FCS together with a kinetic analysis of traces that we presented in this thesis provides a tool to study the essential feature of cotranslational folding that remained obscure so far - the dynamics of cotranslational intermediates. The relatively broad accessibility to the 
optical setup and modest sample requirements means that a relatively high number of different ribosome nascent chain complexes can be measured. This creates conditions for studying a number of various folds and folding intermediates to ascertain the range of kinetically accessible states at each folding event. The combination of PET-FCS together with FPA methods each use a single fluorophore that can be easily introduced at the $\mathrm{N}$-terminus of every protein. The minimally invasive reporter could be used with proteins where structure is unknown and it would provide information on the changes in dynamics during translation.

The relatively recent advances in cotranslational folding and relatively few examples of proteins folding on the ribosome means that a unifying theory of cotranslational folding is absent. In such a situation every experimental finding must be interrogated on whether this is a unique feature for a particular protein or whether a finding is a systematic feature of this biological process. Future studies will have to focus on ways to study cotranslational folding more systematically to disentangle unique protein folding features from the underpinning principles of cotranslational folding. Building a comprehensive cotranslational folding model will allow us to interrogate what kind of deviations from this model result in folding aberrations, protein aggregation and diseases (Powers et al., 2009; Santra et al., 2019). The combined PET-FCS and FPA approaches presented in this thesis will serve to continue to investigate these questions. 


\section{SECTION 5 - Materials and Methods}

\section{HemK constructs for PET and FPA}

The HemK (methyl transferase HemK) coding sequence (ECBD_2409, 834 p, 277aa) was derived from the pET-24a vector(Holtkamp et al., 2015). For PET measurements, Trp at position 78 and - where indicated - Trp6 were mutated to Phe either in the wt or the 4xA HemK NTD sequence (Table 6). The looped construct was generated using Gibson assembly reaction protocol (Gibson et al., 2010; Gibson et al., 2009) introducing five additional glycines in the loop between helix 3 and helix 4, before the wt Gly at position 43 (Table 6). Primers used to generate the glycine insert:

forward 5'-CTCGCCTTTGGCGGCGGC

reverse 5'-GCGTTTCACCGCCGCCGCC

primers used to linearize the vector:

forward 5'-GGCGGCGGCGGCGGCGGTGAAACGCAGCT

reverse 5'-AAAGGCGAGGATAAAAGTACGCCCTTTGCC.

mRNA transcription templates were generated for all lengths using universal commercially available T7 forward primer (Eurofins Genomics, Ebersberg) and three unique reverse primers for the required constructs:

70 (5'-ATGAGCAATGGGTTCACCATCG),

102 (5'-TGCCTGCTCCACCAGACACTCC),

112 (5'-ACGGCAAGGTTGTTCAGGCA) (Eurofins Genomics, Ebersberg).

Table 6 PET-FCS constructs

Amino acid sequences $\mathrm{N}$ to $\mathrm{C}$ terminus

\begin{tabular}{|c|c|}
\hline HemK & $\mathrm{N}$ - to $\mathrm{C}$-terminus $112 \mathrm{aa}$ constructs \\
\hline wt & $\begin{array}{l}\text { MEFQHWLREA ISQLQASESP RRDAEILLEH VTGKGRTFIL AFGETQLTDE } \\
\text { QCQQLDALLT RRRDGEPIAH LTGVREFFSL PLFVSPATLI PRPDTECLVE } \\
\text { QALARLPEQP CR }\end{array}$ \\
\hline $\begin{array}{c}\text { wt } \\
\text { W6F }\end{array}$ & $\begin{array}{l}\text { MEFQHFLREA ISQLQASESP RRDAEILLEH VTGKGRTFIL AFGETQLTDE } \\
\text { QCQQLDALLT RRRDGEPIAH LTGVREFFSL PLFVSPATLI PRPDTECLVE } \\
\text { QALARLPEQP CR }\end{array}$ \\
\hline looped & $\begin{array}{l}\text { MEFQHFLREA ISQLQASESP RRDAEILLEH VTGKGRTFIL AFGGGGGGET } \\
\text { QLTDEQCQQL DALLTRRRDG EPIAHLTGVR EFFSLPLFVS PATLIPRPDT } \\
\text { ECLVEQALAR LPEQPCR }\end{array}$ \\
\hline $4 \times A$ & $\begin{array}{l}\text { MEFQHWLREA ISQLQASESP RRDAEIAAEH VTGKGRTFIL AFGETQLTDE } \\
\text { QCQQADAALT RRRDGEPIAH LTGVREFFSL PLFVSPATLI PRPDTECLVE } \\
\text { QALARLPEQP CR }\end{array}$ \\
\hline $\begin{array}{l}4 \times A \\
W 6 F\end{array}$ & $\begin{array}{l}\text { MEFQHFLREA ISQLQASESP RRDAEIAAEH VTGKGRTFIL AFGETQLTDE } \\
\text { QCQQADAALT RRRDGEPIAH LTGVREFFSL PLFVSPATLI PRPDTECLVE } \\
\text { QALARLPEQP CR }\end{array}$ \\
\hline
\end{tabular}


The FPA reporters contained a fragments of wt or 4xA HemK (aa 1-101) followed by the 17 aa SecM stalling peptide (Nakatogawa and Ito, 2002), and a fragment (aa 1-20) of cold shock protein A (CspA) (UniProt ID: P0A9X9), all truncations shown in Table 7. The full-length plasmids were synthesized by Eurofins Genomics (Ebersberg, Germany) using a pEX-A128 vector carrying a kanamycin resistance cassette. C-terminal HemK truncations were performed via a cloning protocol that involved vector linearization during PCR (all primer sequences available upon request). All generated constructs were verified by Sanger sequencing (Microsynth AG, Göttingen, Germany). mRNA transcription templates were generated for all FPA constructs using universal T7 forward and CspA reverse primer (5'AGGAGTGATGAAGCCGAAGCCT) (Eurofins Genomics, Ebersberg).

\section{Table 7 Force profile constructs of full-length wt HemK}

Amino acid sequence from $\mathrm{N}$ - to $\mathrm{C}$-terminus, superscript numbers indicate construct truncations in HemK length.

\begin{tabular}{|c|c|c|}
\hline HemK & SecM & CspA \\
\hline 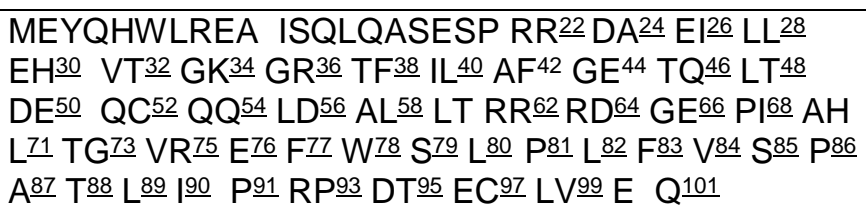 & $\begin{array}{l}\text { FSTPVWIS } \\
\text { QAQGIRAGP }\end{array}$ & $\begin{array}{l}\text { MSGKMTGIVK } \\
\text { WFNADKGFGFITP }\end{array}$ \\
\hline
\end{tabular}

All mRNAs lacked a stop codon and were transcribed in vitro in buffer $(40 \mathrm{mM}$ Tris- $\mathrm{HCl}, \mathrm{pH}$ 7.5, $15 \mathrm{mM} \mathrm{MgCl}_{2}, 2 \mathrm{mM}$ spermidine, $10 \mathrm{mM} \mathrm{NaCl}, 10 \mathrm{mM}$ DTT, $5 \mathrm{mM}$ GMP). The DNA template $(10 \%(\mathrm{v} / \mathrm{v}))$ was incubated with $3 \mathrm{mM}$ each of GTP, ATP, CTP and UTP, pyrophosphatase (5 u/ml), RiboLock RNase inhibitor (1.5\% (v/v), Fermentas), and T7 RNApolymerase $(1.6 \mathrm{u} / \mu \mathrm{L})$, for $4 \mathrm{~h}$ at $37^{\circ} \mathrm{C}$. The mRNA was purified by anion exchange chromatography on a HiTrap Q HP column (GE Healthcare) operated on Äkta FPLC system in buffer $30 \mathrm{mM}$ Bis-Tris pH 6, $1 \mathrm{mM}$ EDTA, $300 \mathrm{mM} \mathrm{NaCl}$. mRNA was eluted using a linear gradient from $300 \mathrm{mM}$ to $1.5 \mathrm{M} \mathrm{NaCl}$ over 20 column volumes. The mRNA-containing fractions were pooled, the mRNAs precipitated with ethanol and the mRNAs pelleted by centrifugation at $4000 \mathrm{~g}$ for $1 \mathrm{~h}$ at $4^{\circ} \mathrm{C}$. The mRNA pellets were resuspended in RNase- and DNase-free water and the concentration was measured using Nanodrop 2000c (Thermo Scientific). $8 \mathrm{M}$ urea $10 \%$ polyacrylamide gel electrophoresis was used for mRNA quality control.

\section{In vitro translation}

Translation components, including 70 S ribosomes, initiation factors, elongation factors (EF-G and EF-Tu) and total aminoacyl-tRNAs (aa-tRNAs) were prepared as described (Doerfel et al., 
2013; Holtkamp et al., 2015; Milon et al., 2007; Mittelstaet et al., 2013; Rodnina and Wintermeyer, 1995). Initiation complexes were formed in buffer A (50 mM HEPES pH 7.5, 70 $\mathrm{mM} \mathrm{NH}_{4} \mathrm{Cl}, 30 \mathrm{mM} \mathrm{KCl}, 7 \mathrm{mM} \mathrm{MgCl}$, with $2 \mathrm{mM}$ DTT, and $\left.2 \mathrm{mM} \mathrm{GTP}\right)$. Ribosomes (0.5 $\left.\mu \mathrm{M}\right)$ were incubated with initiation factors (IF1, IF2, and IF3; $2.25 \mu \mathrm{M}$ each), mRNA (1.5 $\mu \mathrm{M})$, and

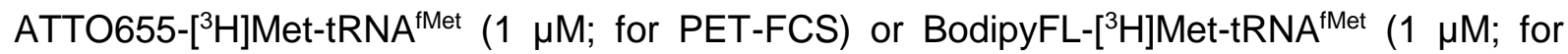
arrest peptide assay) for $45 \mathrm{~min}$ at $37^{\circ} \mathrm{C}$. Fluorescence-labeled tRNAs were prepared as described (Mittelstaet et al., 2013). EF-Tu-GTP was prepared in buffer A by incubating EFTu-GDP $(120 \mu \mathrm{M})$ with phosphoenol pyruvate $(3 \mathrm{mM})$ pyruvate kinase $(0.05 \mathrm{mg} / \mathrm{mL})$ for 15 $\min$ at $37^{\circ} \mathrm{C}$. The ternary complex EF-Tu-GTP-aa-tRNA was formed by adding total aminoacyl-tRNA $(200 \mu \mathrm{M})$ to EF-Tu-GTP followed by a $1 \mathrm{~min}$ incubation at $37^{\circ} \mathrm{C}$.

All in vitro translation reactions were performed in buffer $\mathrm{B}(50 \mathrm{mM}$ HEPES pH 7.5, $70 \mathrm{mM}$ $\mathrm{NH}_{4} \mathrm{Cl}, 30 \mathrm{mM} \mathrm{KCl}, 3.5 \mathrm{mM} \mathrm{MgCl}$, $1 \mathrm{mM}$ DTT, $0.5 \mathrm{mM}$ spermidine and $8 \mathrm{mM}$ putrescine). Initiation complexes (40 nM) were mixed with EF-Tu-GTP-aa-tRNA $(50 \mu \mathrm{M})$ and EF-G $(1 \mu \mathrm{M})$, and incubated for $5 \mathrm{~min}$ at $37^{\circ} \mathrm{C}$. All mRNAs lacked the final stop codon and produced RNCs stalled with a peptidyl-tRNA in the $\mathrm{P}$ site. For PET-FCS, the RNCs were purified from translation factors and unbound fluorescence-labeled tRNA by sucrose cushion centrifugation using 2.2 $\mathrm{M}$ sucrose in buffer $\mathrm{B}$. The ribosomes were pelleted using the TLA-100 rotor (Beckman Coulter) at $68,000 \mathrm{rpm}$ for $40 \mathrm{~min}$ at $4^{\circ} \mathrm{C}$. The pellet containing RNC was resuspended in buffer $B$, and the RNC concentration was determined by liquid-liquid radioactivity counting of ${ }^{3} \mathrm{H}$-labeled Met. The complexes were flash frozen in liquid nitrogen and stored in $-80^{\circ} \mathrm{C}$ until use. Translation efficiency was monitored on Tris-tricine SDS PAGE.

All translation samples were prepared for Tris-tricine SDS PAGE as follows. The nascent chains were released from the ribosome by adding $1.5 \mathrm{M}$ hydroxylamine and incubating the samples for $1 \mathrm{~h}$ at $37^{\circ} \mathrm{C}$. The samples were then diluted with gel loading buffer (50 mM Tris$\mathrm{HCl} \mathrm{pH} \mathrm{6.8,4 \%} \mathrm{w/v} \mathrm{SDS,} 2 \% \mathrm{v} / \mathrm{v} 2$-mercaptoethanol and 12\% w/v glycerol) and translation products were separated using Tris-Tricine SDS-PAGE (Schagger, 2006). A 16.5\% separating gel $(49.5 \% \mathrm{~T}, 6 \% \mathrm{C}), 10 \%$ spacer gel and $4 \%$ stacking gel were used. The in vitro translation products were visualized by detecting the N-terminal dye using a Fujifilm FLA-9000 fluorescence scanner equipped with a laser of $488 \mathrm{~nm}$ wavelength to detect Bodipy FL, or the $680 \mathrm{~nm}$ laser to detect ATTO-655. The band intensities on the gel were quantified and analyzed using LI-COR Biosciences $\mathrm{GmbH}$ Image Studio version 5.2.5. The fraction of fulllength product was calculated by dividing the full-length band intensity by the sum of the fulllength and arrested band intensities. 
To produce free peptide, nascent chains were released from the ribosome with hydroxylamine. RNC was incubated with hydroxylamine $(5 \% \mathrm{w} / \mathrm{v}$; $\mathrm{pH} \leq 8)$ for $1 \mathrm{~h}$ at $37^{\circ} \mathrm{C}$. This was followed by a sucrose cushion centrifugation as above. The supernatant containing the released nascent chains was collected for PET-FCS measurements.

\section{Fluorescence correlation spectroscopy}

Fluorescence correlation measurements were performed using the MicroTime 200 system (PicoQuant, Berlin, Germany), which is based on a modified Olympus IX 73 confocal microscope and equipped with a water objective lens with 60x magnification and 1.2 N.A. (Olympus UPlanSApo). For excitation, a collimated laser (LDH-D-C-640, PicoQuant GmbH) beam with $636.5 \mathrm{~nm}$ wavelength (operated in continuous wave mode) with large diameter was focused through the objective into the sample solution. The laser power was set to $\sim 40 \mu \mathrm{W}$ to minimize Atto655 triplet state formation and to avoid photobleaching. Fluorescence signals were collected using the same objective (epifluorescence configuration) and separated from the excitation light by a dichroic mirror. After that, the collected fluorescence light was focused through a $50 \mu \mathrm{m}$ pinhole to eliminate fluorescence coming from axial positions away from the focal plane (confocal detection). A 50/50 beam splitter was used to split the fluorescence signal into two channels, where light was focused onto two single-photon avalanche photodiodes (SPAD) after passing through a band pass filter $(690 / 70 \mathrm{~nm})$. The signals of two SPADs were cross-correlated to eliminate SPAD after-pulsing effects.

Purified RNCs were measured at $\sim 4 \mathrm{nM}$ in buffer $\mathrm{B}$, and sample concentration was adjusted in such a way as to yield an average of one molecule within the confocal detection volume for all measurements. For the Trp titrations, ACFs were recorded for purified RNCs of HemK 70 W6F and HemK 102 W6F at different concentrations of added Trp. A solution of $70 \mathrm{mM}$ Trp was prepared in buffer $B$, and final free Trp concentrations ranged from $1.8 \mathrm{mM}$ to $45 \mathrm{mM}$. The recorded ACFs were then fitted (Equation 1), and the parameters obtained were processed as described below. Measurements were performed at ambient temperature $\left(22^{\circ} \mathrm{C}\right)$. For each RNC solution, single-photon fluorescence detection events were recorded for at least four consecutive time intervals of $10 \mathrm{~min}$. The auto-correlation functions (ACF) were computed using the SymPhoTime 64 software (PicoQuant). After normalization, these ACFs were compared to confirm that the RNCs were stable throughout the duration of the measurement, and the technical replicates were averaged. The experiments were repeated a minimum of 23 times for the same class of RNCs from different preparations. 
The microscope is setup with a detection volume where the lateral dimension is much larger than the horizontal dimension, therefore fitting of initial ACFs was carried out using a model for $2 \mathrm{D}$ single species diffusion with two relaxation rate constants, a triplet rate constant, and a diffusion rate constant,

$G(\tau)=\left(1+c_{1} e^{-k_{1} t}+c_{2} e^{-k_{2} t}\right)\left(\frac{1-F+F e^{-k_{f} t}}{1-F}\right)\left(\frac{1}{N}\right)\left(1+k_{d} t\right)^{-1} \quad$ Equation 1

where $k_{1}$ and $k_{2}$ are apparent relaxation rate constants with respective amplitudes $c_{1}$ and $c_{2}$, $N$ is the average number of molecules in the confocal volume, $F$ is the amplitude for the triplet component with rate constant $k_{f}$, and $k_{d}$ is the inverse diffusion time.

\section{Kinetic modeling}

To fit the ACFs of the PET-FCS measurements in the commercial KinTek software, the diffusion and triplet state components were subtracted from each curve using the respective fitted parameters (Equation 1; Table 2). The software KinTek Global Kinetic Explorer V 6.3 was used for kinetic modelling (Johnson et al., 2009a, b). In all cases, the exponential decays of different PET quenching curves were simulated by the KinTek software as a sum of species $C, D, R$, and W (see text) for a given HemK construct, multiplied by a species-specific amplitude coefficient. The amplitude coefficients were assumed to be identical for all simulated traces in a particular dataset.

The dominant Trp quenching mechanism for ATTO655 is through static quenching due to stacking interactions in the fluorophore-quencher pair; the dissociation rate of the quenched complex is defined by the specific properties of these stacking interactions (Limpouchova and Prochazka, 2016; Sharma et al., 2017). Therefore, the dissociation rate (dequenching) constant of the ATTO655-Trp complex is expected to be independent of the source of Trp or the structure of the RNC. To estimate the $k_{\text {off }}$ for the ATTO655-Trp complex, we measured ACFs for HemK70 W6F and HemK102 W6F RNCs with increasing concentrations of free Trp in solution (Figure 15). Each RNC construct contained 7 ACFs for each tryptophan concentration of $[\operatorname{Trp}]=0,1.8,4.5,9,18,27,45 \mathrm{mM}$ (dataset $A$ ), and each of these data curves contained the average of two independent replicates. The amplitudes of reactions in the ns and $\mu$ s time domain increased with free Trp concentrations.

To determine the dequenching constant, we globally fit the titration dataset $A$ to the kinetic model (Figure 15) after introducing a term that is concentration-dependent (Trp binding to ATTO655), while all other rate constants remained concentration-independent and constant in 
all of these experiments. Global fitting of the data yielded a $2.0 \pm 0.1 \mu \mathrm{s}^{-1}$ dequenching rate constant of the ATTO655-Trp pair (Table 3). Other rates were not sufficiently constrained by the Trp titration dataset, as evident from large standard errors (Table 3), and were not used in the following fitting.

In the second step, we locked the ATTO655-Trp dissociation rate at $2 \mu \mathrm{s}^{-1}$ and globally fit the experimental dataset for different RNCs: 70, 102, 112 aa long HemK wt with Trp (W6) and without Trp (W6F), the 70 and 112 HemK 4xA W6 and W6F, HemK 102 loop W6F and HemK 112 loop W6F curves (dataset B) ( Figure 18). There were 16 unlinked independent rates. The $W$ state dequenching $k_{\text {off }}$ value was locked at $2 \mu \mathrm{s}^{-1}$. The $k_{\text {off }}$ rate from state $R$ were coupled across all constructs, as this rate also depend on the properties of the dye-quenching pair, which should be uniform across the different complexes. In the case of HemK112 4xA, all rates were separated from other constructs except the $k_{\text {off }}$ of $R$ state. The rates of each step were linked between HemK70 wt and 4xA. The loop construct $R$ state $k_{\text {on }}$ rates were coupled to the corresponding wt construct $R$ state $k_{\text {on }}$ rates. For all constructs (except $1124 \times A$ ), state $C$ to $R$ and state $\mathrm{C}$ to $\mathrm{W} \mathrm{k}_{\text {on }}$ were also linked, due to similarity. All standard errors reported in Table 3 and Table 4 were calculated using a covariance matrix derived using nonlinear regression algorithms (Johnson et al., 2009b). Using the Kintek Explorer software the fit quality of the entire dataset was subject to a confidence contour analysis to evaluate the numerical space over which the parameters could vary and still provide high quality fit (Johnson et al., 2009a). The analysis is based on measuring the dependence of the sum square error on a pair of given parameters while the remaining parameters are left free to vary while producing the best fit (Table 8) (Johnson et al., 2009a).

\section{Calculations of the transition state energy barrier}

Transition state theory (Fersht et al., 1999) was used to calculate the energy barrier between the conformational state ensembles of different HemK constructs. The $k_{\text {on }}$ and rates of conformational fluctuations between states that were obtained from the kinetic modelling were used to solve for the transition state $\left(\Delta G^{\ddagger}\right)$ energy barrier between the states (Baryshnikova et al., 2005; Fersht et al., 1999)

$$
k_{\text {on }}=\mathbf{k}_{\mathrm{B}} \frac{\mathrm{T}}{\mathrm{h}} \boldsymbol{\kappa} \cdot \exp \left(\frac{-\Delta \mathrm{G}_{\neq-\mathrm{D}}}{\mathrm{RT}}\right) \quad \text { Equation } 2
$$

where $R$ is the gas constant $8.3145 \mathrm{~J} \mathrm{~mol}^{-1} ; T$ is temperature $\left(295^{\circ} \mathrm{K}\right) ; \mathrm{K}$ is transmission coefficient (approximated to 1.0 in transition state theory) (Fersht et al., 1999); $k_{B}$ is Boltzmann's constant $1.38 \times 10^{-23} \mathrm{~J} \mathrm{~K}^{-1}$; and $h$ is Planck's constant $6.6 \times 10^{-34} \mathrm{~m}^{2} \mathrm{~kg} \mathrm{~s}^{-1}$. 
Table 8 Upper and lower boundaries of the kinetic fit

Boundaries at $\mathrm{minChi}^{2} / \mathrm{Chi}^{2}$ threshold 0.8333 , cells coded in the same shade were linked during fitting, values in red were locked.

\begin{tabular}{|c|c|c|c|c|}
\hline Construct & Elemental Rate & Best-fit Value & Lower Boundary & Upper Boundary \\
\hline \multirow[t]{8}{*}{$70 w t$} & $\mathrm{k}_{\text {on }}(\mathrm{D} \leftrightarrow \mathrm{R})$ & 21.8 & 17.4 & 28.9 \\
\hline & $\mathrm{k}_{\text {on }}(\mathrm{C} \leftrightarrow \mathrm{R})$ & 0.372 & 0.354 & 0.389 \\
\hline & $\mathrm{k}_{\text {off }}(\mathrm{D} / \mathrm{C} \leftrightarrow \mathrm{R})$ & 0.309 & 0.247 & 0.458 \\
\hline & $\mathrm{k}_{\text {on }}(\mathrm{D} \leftrightarrow \mathrm{W})$ & 4.24 & 2.64 & 6.29 \\
\hline & $\mathrm{k}_{\text {on }}(\mathrm{C} \leftrightarrow \mathrm{W})$ & 0.0725 & 0.0441 & 0.102 \\
\hline & $\mathrm{k}_{\text {off }}(\mathrm{D} / \mathrm{C} \leftrightarrow \mathrm{W})$ & 2 & $n / a$ & $n / a$ \\
\hline & $\mathrm{k}_{\text {on }}(\mathrm{D} \leftrightarrow \mathrm{C})$ & 0.976 & 0.45 & 2.83 \\
\hline & $\mathrm{k}_{\text {off }}(\mathrm{D} \leftrightarrow C)$ & 0.0167 & 0.00846 & 0.0391 \\
\hline \multirow[t]{8}{*}{$704 \times A$} & $k_{\text {on }}(D \leftrightarrow R)$ & 21.8 & 17.4 & 28.9 \\
\hline & $\mathrm{k}_{\text {on }}(\mathrm{C} \leftrightarrow \mathrm{R})$ & 0.372 & 0.354 & 0.389 \\
\hline & $\mathrm{k}_{\text {off }}(\mathrm{D} / \mathrm{C} \leftrightarrow \mathrm{R})$ & 0.309 & 0.247 & 0.458 \\
\hline & $\mathrm{k}_{\text {on }}(\mathrm{D} \leftrightarrow \mathrm{W})$ & 4.24 & 2.64 & 6.29 \\
\hline & $\mathrm{k}_{\text {on }}(\mathrm{C} \leftrightarrow \mathrm{W})$ & 0.0725 & 0.0441 & 0.102 \\
\hline & $\mathrm{k}_{\text {off }}(\mathrm{D} / \mathrm{C} \leftrightarrow \mathrm{W})$ & 2 & $n / a$ & $n / a$ \\
\hline & $\mathrm{k}_{\text {on }}(\mathrm{D} \leftrightarrow \mathrm{C})$ & 0.976 & 0.45 & 2.83 \\
\hline & $\mathrm{k}_{\text {off }}(\mathrm{D} \leftrightarrow \mathrm{C})$ & 0.0167 & 0.00846 & 0.0391 \\
\hline \multirow[t]{8}{*}{$102 \mathrm{wt}$} & $\mathrm{k}_{\text {on }}(\mathrm{D} \leftrightarrow \mathrm{R})$ & 25 & 20 & 35.2 \\
\hline & $\mathrm{k}_{\text {on }}(\mathrm{C} \leftrightarrow \mathrm{R})$ & 0.372 & 0.354 & 0.389 \\
\hline & $\mathrm{k}_{\text {off }}(\mathrm{D} / \mathrm{C} \leftrightarrow \mathrm{R})$ & 0.309 & 0.247 & 0.458 \\
\hline & $\mathrm{k}_{\text {on }}(\mathrm{D} \leftrightarrow \mathrm{W})$ & & & \\
\hline & $\mathrm{k}_{\text {on }}(\mathrm{C} \leftrightarrow \mathrm{W})$ & 0.0725 & 0.0441 & 0.102 \\
\hline & $\mathrm{k}_{\text {off }}(\mathrm{D} / \mathrm{C} \leftrightarrow \mathrm{W})$ & 2 & $\mathrm{n} / \mathrm{a}$ & $\mathrm{n} / \mathrm{a}$ \\
\hline & $\mathrm{k}_{\text {on }}(\mathrm{D} \leftrightarrow \mathrm{C})$ & 0.133 & $7.71 \mathrm{e}-007$ & 1.84 \\
\hline & $\mathrm{k}_{\text {off }}(\mathrm{D} \leftrightarrow \mathrm{C})$ & 0.00198 & $1.3 e-008$ & 0.0245 \\
\hline \multirow[t]{5}{*}{102 loop } & $\mathrm{k}_{\text {on }}(\mathrm{D} \leftrightarrow \mathrm{R})$ & 25 & 20 & 35.2 \\
\hline & $\mathrm{k}_{\text {on }}(\mathrm{C} \leftrightarrow \mathrm{R})$ & 0.372 & 0.354 & 0.389 \\
\hline & $\mathrm{k}_{\text {off }}(\mathrm{D} / \mathrm{C} \leftrightarrow \mathrm{R})$ & 0.309 & 0.247 & 0.458 \\
\hline & $\mathrm{k}_{\text {on }}(\mathrm{D} \leftrightarrow \mathrm{C})$ & 4.4 & 3.52 & 7.73 \\
\hline & $\mathrm{k}_{\text {off }}(\mathrm{D} \leftrightarrow \mathrm{C})$ & 0.0653 & 0.0555 & 0.0885 \\
\hline
\end{tabular}




\begin{tabular}{|c|c|c|c|c|}
\hline \multirow[t]{5}{*}{112 loop } & $\mathrm{k}_{\text {on }}(\mathrm{D} \leftrightarrow \mathrm{R})$ & 29 & 23.2 & 40.7 \\
\hline & $\mathrm{k}_{\text {on }}(\mathrm{C} \leftrightarrow \mathrm{R})$ & 0.372 & 0.354 & 0.389 \\
\hline & $\mathrm{k}_{\text {off }}(\mathrm{D} / \mathrm{C} \leftrightarrow \mathrm{R})$ & 0.309 & 0.247 & 0.458 \\
\hline & $\mathrm{k}_{\text {on }}(\mathrm{D} \leftrightarrow \mathrm{C})$ & 3.44 & 2.75 & 6 \\
\hline & $\mathrm{k}_{\text {off }}(\mathrm{D} \leftrightarrow C)$ & 0.0442 & 0.0376 & 0.0659 \\
\hline \multirow[t]{8}{*}{$112 \mathrm{wt}$} & $\mathrm{k}_{\text {on }}(\mathrm{D} \leftrightarrow \mathrm{R})$ & 29 & 23.2 & 40.7 \\
\hline & $\mathrm{k}_{\text {on }}(\mathrm{C} \leftrightarrow \mathrm{R})$ & 0.372 & 0.354 & 0.389 \\
\hline & $\mathrm{k}_{\text {off }}(\mathrm{D} / \mathrm{C} \leftrightarrow \mathrm{R})$ & 0.309 & 0.247 & 0.458 \\
\hline & $\mathrm{k}_{\text {on }}(\mathrm{D} \leftrightarrow \mathrm{W})$ & 5.64 & 3.41 & 8.82 \\
\hline & $\mathrm{k}_{\mathrm{on}}(\mathrm{C} \leftrightarrow \mathrm{W})$ & 0.0725 & 0.0441 & 0.102 \\
\hline & $\mathrm{k}_{\text {off }}(\mathrm{D} / \mathrm{C} \leftrightarrow \mathrm{W})$ & 2 & $\mathrm{n} / \mathrm{a}$ & $\mathrm{n} / \mathrm{a}$ \\
\hline & $\mathrm{k}_{\text {on }}(\mathrm{D} \leftrightarrow \mathrm{C})$ & 0.000179 & $1.78 \mathrm{e}-008$ & 1.6 \\
\hline & $\mathrm{k}_{\mathrm{off}}(\mathrm{D} \leftrightarrow \mathrm{C})$ & $2.29 e-006$ & $2.29 \mathrm{e}-010$ & 0.0182 \\
\hline \multirow[t]{8}{*}{$1124 \times A$} & $\mathrm{k}_{\mathrm{on}}(\mathrm{D} \leftrightarrow \mathrm{R})$ & 24.1 & 17.4 & 40 \\
\hline & $\mathrm{k}_{\mathrm{on}}(\mathrm{C} \leftrightarrow \mathrm{R})$ & 0.346 & 0.329 & 0.361 \\
\hline & $\mathrm{k}_{\text {off }}(\mathrm{D} / \mathrm{C} \leftrightarrow \mathrm{R})$ & 0.309 & 0.247 & 0.458 \\
\hline & $\mathrm{k}_{\mathrm{on}}(\mathrm{D} \leftrightarrow \mathrm{W})$ & 4.7 & 2.9 & 8.26 \\
\hline & $\mathrm{k}_{\text {on }}(\mathrm{C} \leftrightarrow \mathrm{W})$ & 0.0674 & 0.041 & 0.0948 \\
\hline & $\mathrm{k}_{\text {off }}(\mathrm{D} / \mathrm{C} \leftrightarrow \mathrm{W})$ & 2 & $\mathrm{n} / \mathrm{a}$ & $\mathrm{n} / \mathrm{a}$ \\
\hline & $\mathrm{k}_{\text {on }}(\mathrm{D} \leftrightarrow \mathrm{C})$ & 1.61 & 0.661 & 4.68 \\
\hline & $\mathrm{k}_{\mathrm{off}}(\mathrm{D} \leftrightarrow \mathrm{C})$ & 0.0231 & 0.0116 & 0.0454 \\
\hline
\end{tabular}




\section{References}

Adio, S., Sharma, H., Senyushkina, T., Karki, P., Maracci, C., Wohlgemuth, I., Holtkamp, W., Peske, F., and Rodnina, M.V. (2018). Dynamics of ribosomes and release factors during translation termination in E. coli. Elife 7 .

Agashe, V.R., Shastry, M.C., and Udgaonkar, J.B. (1995). Initial hydrophobic collapse in the folding of barstar. Nature 377, 754-757.

Alexander, L.M., Goldman, D.H., Wee, L.M., and Bustamante, C. (2019). Non-equilibrium dynamics of a nascent polypeptide during translation suppress its misfolding. Nat Commun 10 , 2709.

Anfinsen, C.B. (1973). Principles That Govern Folding of Protein Chains. Science 181, $223-$ 230.

Anfinsen, C.B., Haber, E., Sela, M., and White, F.H., Jr. (1961). The kinetics of formation of native ribonuclease during oxidation of the reduced polypeptide chain. Proc Natl Acad Sci U S A 47, 1309-1314.

Bai, Y., Sosnick, T.R., Mayne, L., and Englander, S.W. (1995). Protein folding intermediates: native-state hydrogen exchange. Science 269, 192-197.

Baker, D., and Agard, D.A. (1994). Kinetics versus thermodynamics in protein folding. Biochemistry 33, 7505-7509.

Baldwin, R.L., and Rose, G.D. (1999a). Is protein folding hierarchic? I. Local structure and peptide folding. Trends Biochem Sci 24, 26-33.

Baldwin, R.L., and Rose, G.D. (1999b). Is protein folding hierarchic? II. Folding intermediates and transition states. Trends Biochem Sci 24, 77-83.

Bars, I., and Terning, J. (2010). Extra Dimensions in Space and Time. Multiversal Journeys, 1-217.

Baryshnikova, E.N., Melnik, B.S., Finkelstein, A.V., Semisotnov, G.V., and Bychkova, V.E. (2005). Three-state protein folding: experimental determination of free-energy profile. Protein Sci 14, 2658-2667.

Bashford, D., Cohen, F.E., Karplus, M., Kuntz, I.D., and Weaver, D.L. (1988). Diffusioncollision model for the folding kinetics of myoglobin. Proteins 4, 211-227.

Beechem, J.M., Sherman, M.A., and Mas, M.T. (1995). Sequential domain unfolding in phosphoglycerate kinase: fluorescence intensity and anisotropy stopped-flow kinetics of several tryptophan mutants. Biochemistry 34, 13943-13948.

Belardinelli, R., Sharma, H., Peske, F., Wintermeyer, W., and Rodnina, M.V. (2016). Translocation as continuous movement through the ribosome. RNA Biol 13, 1197-1203. 
Ben-Shem, A., Jenner, L.B., Yusupova, G., and Yusupov, M. (2011). Crystal structure of the eukaryotic 80 S ribosome. In Ribosomes: Structure, Function, and Dynamics, M.V. Rodnina, W. Wintermeyer, and R. Green, eds. (Vienna: Springer Vienna), pp. 75-81.

Best, R.B., and Clarke, J. (2002). What can atomic force microscopy tell us about protein folding? Chem Commun (Camb), 183-192.

Bhushan, S., Gartmann, M., Halic, M., Armache, J.P., Jarasch, A., Mielke, T., Berninghausen, O., Wilson, D.N., and Beckmann, R. (2010). alpha-Helical nascent polypeptide chains visualized within distinct regions of the ribosomal exit tunnel. Nature structural \& molecular biology 17, 313-317.

Buhr, F., Jha, S., Thommen, M., Mittelstaet, J., Kutz, F., Schwalbe, H., Rodnina, M.V., and Komar, A.A. (2016). Synonymous Codons Direct Cotranslational Folding toward Different Protein Conformations. Mol Cell 61, 341-351.

Cabrita, L.D., Cassaignau, A.M.E., Launay, H.M.M., Waudby, C.A., Wlodarski, T., Camilloni, C., Karyadi, M.E., Robertson, A.L., Wang, X., Wentink, A.S., et al. (2016). A structural ensemble of a ribosome-nascent chain complex during cotranslational protein folding. Nature structural \& molecular biology 23, 278-285.

Canet, D., Doering, K., Dobson, C.M., and Dupont, Y. (2001). High-sensitivity fluorescence anisotropy detection of protein-folding events: application to alpha-lactalbumin. Biophysical journal 80, 1996-2003.

Choi, I.G., and Kim, S.H. (2006). Evolution of protein structural classes and protein sequence families. Proc Natl Acad Sci U S A 103, 14056-14061.

Chung, H.S., McHale, K., Louis, J.M., and Eaton, W.A. (2012). Single-molecule fluorescence experiments determine protein folding transition path times. Science 335, 981-984.

Dagan, S., Hagai, T., Gavrilov, Y., Kapon, R., Levy, Y., and Reich, Z. (2013). Stabilization of a protein conferred by an increase in folded state entropy. Proceedings of the National Academy of Sciences of the United States of America 110, 10628-10633.

Daggett, V., and Fersht, A.R. (2003). Is there a unifying mechanism for protein folding? Trends Biochem Sci 28, 18-25.

Deckert, A., Waudby, C.A., Wlodarski, T., Wentink, A.S., Wang, X., Kirkpatrick, J.P., Paton, J.F., Camilloni, C., Kukic, P., Dobson, C.M., et al. (2016). Structural characterization of the interaction of alpha-synuclein nascent chains with the ribosomal surface and trigger factor. Proc Natl Acad Sci U S A 113, 5012-5017.

Deniz, A.A., Laurence, T.A., Beligere, G.S., Dahan, M., Martin, A.B., Chemla, D.S., Dawson, P.E., Schultz, P.G., and Weiss, S. (2000). Single-molecule protein folding: diffusion fluorescence resonance energy transfer studies of the denaturation of chymotrypsin inhibitor 2. Proc Natl Acad Sci U S A 97, 5179-5184. 
Dever, T.E., Dinman, J.D., and Green, R. (2018). Translation Elongation and Recoding in Eukaryotes. Cold Spring Harb Perspect Biol 10.

Dill, K.A., Bromberg, S., Yue, K., Fiebig, K.M., Yee, D.P., Thomas, P.D., and Chan, H.S. (1995). Principles of protein folding--a perspective from simple exact models. Protein Sci 4, 561-602.

Dobson, C.M. (2019). Biophysical Techniques in Structural Biology. Annu Rev Biochem 88, 25-33.

Doerfel, L.K., Wohlgemuth, I., Kothe, C., Peske, F., Urlaub, H., and Rodnina, M.V. (2013). EF$P$ is essential for rapid synthesis of proteins containing consecutive proline residues. Science 339, 85-88.

Dolgikh, D.A., Gilmanshin, R.I., Brazhnikov, E.V., Bychkova, V.E., Semisotnov, G.V., Venyaminov, S., and Ptitsyn, O.B. (1981). Alpha-Lactalbumin: compact state with fluctuating tertiary structure? FEBS letters 136, 311-315.

Doose, S., Neuweiler, H., Barsch, H., and Sauer, M. (2007). Probing polyproline structure and dynamics by photoinduced electron transfer provides evidence for deviations from a regular polyproline type II helix. Proc Natl Acad Sci U S A 104, 17400-17405.

Doose, S., Neuweiler, H., and Sauer, M. (2009). Fluorescence quenching by photoinduced electron transfer: a reporter for conformational dynamics of macromolecules. Chemphyschem 10, 1389-1398.

Dyson, H.J., and Wright, P.E. (2004). Unfolded proteins and protein folding studied by NMR. Chem Rev 104, 3607-3622.

Eftink, M.R. (1994). The use of fluorescence methods to monitor unfolding transitions in proteins. Biophysical journal 66, 482-501.

Elson, E.L. (2018). Introduction to fluorescence correlation Spectroscopy-Brief and simple. Methods 140-141, 3-9.

Englander, S.W., and Mayne, L. (2014). The nature of protein folding pathways. Proc Natl Acad Sci U S A 111, 15873-15880.

Fa, M., Karolin, J., Aleshkov, S., Strandberg, L., Johansson, L.B., and Ny, T. (1995). Timeresolved polarized fluorescence spectroscopy studies of plasminogen activator inhibitor type 1: conformational changes of the reactive center upon interactions with target proteases, vitronectin and heparin. Biochemistry 34, 13833-13840.

Fabian, H., and Naumann, D. (2004). Methods to study protein folding by stopped-flow FT-IR. Methods 34, 28-40.

Farias-Rico, J.A., Ruud Selin, F., Myronidi, I., Fruhauf, M., and von Heijne, G. (2018). Effects of protein size, thermodynamic stability, and net charge on cotranslational folding on the ribosome. Proc Natl Acad Sci U S A 115, E9280-E9287. 
Fersht, A., Fersht, U.A., Freeman, W.H., and Company (1999). Structure and Mechanism in Protein Science: A Guide to Enzyme Catalysis and Protein Folding (W. H. Freeman).

Fersht, A.R. (1995). Optimization of rates of protein folding: the nucleation-condensation mechanism and its implications. Proc Natl Acad Sci U S A 92, 10869-10873.

Fersht, A.R. (1997). Nucleation mechanisms in protein folding. Curr Opin Struct Biol 7, 3-9.

Finkelstein, A.V., and Ptitsyn, O.B. (2016a). Lecture 4. In Protein Physics (Second Edition), A.V. Finkelstein, and O.B. Ptitsyn, eds. (Amsterdam: Academic Press), pp. 39-50.

Finkelstein, A.V., and Ptitsyn, O.B. (2016b). Lecture 5. In Protein Physics (Second Edition), A.V. Finkelstein, and O.B. Ptitsyn, eds. (Amsterdam: Academic Press), pp. 51-65.

Finkelstein, A.V., and Ptitsyn, O.B. (2016c). Lecture 17. In Protein Physics (Second Edition), A.V. Finkelstein, and O.B. Ptitsyn, eds. (Amsterdam: Academic Press), pp. 253-273.

Finkelstein, A.V., and Ptitsyn, O.B. (2016d). Lecture 19. In Protein Physics (Second Edition), A.V. Finkelstein, and O.B. Ptitsyn, eds. (Amsterdam: Academic Press), pp. 289-306.

Fu, Z., Kaledhonkar, S., Borg, A., Sun, M., Chen, B., Grassucci, R.A., Ehrenberg, M., and Frank, J. (2016). Key Intermediates in Ribosome Recycling Visualized by Time-Resolved Cryoelectron Microscopy. Structure 24, 2092-2101.

Galzitskaya, O.V., and Finkelstein, A.V. (1995). Folding of chains with random and edited sequences: similarities and differences. Protein Eng 8, 883-892.

Gao, N., Zavialov, A.V., Li, W., Sengupta, J., Valle, M., Gursky, R.P., Ehrenberg, M., and Frank, J. (2005). Mechanism for the disassembly of the posttermination complex inferred from cryo-EM studies. Mol Cell 18,663-674.

Garbuzynskiy, S.O., Ivankov, D.N., Bogatyreva, N.S., and Finkelstein, A.V. (2013). Golden triangle for folding rates of globular proteins. Proc Natl Acad Sci U S A 110, 147-150.

Gershenson, A., Gosavi, S., Faccioli, P., and Wintrode, P.L. (2020). Successes and challenges in simulating the folding of large proteins. J Biol Chem 295, 15-33.

Gibson, D.G., Glass, J.I., Lartigue, C., Noskov, V.N., Chuang, R.Y., Algire, M.A., Benders, G.A., Montague, M.G., Ma, L., Moodie, M.M., et al. (2010). Creation of a bacterial cell controlled by a chemically synthesized genome. Science 329, 52-56.

Gibson, D.G., Young, L., Chuang, R.Y., Venter, J.C., Hutchison, C.A., 3rd, and Smith, H.O. (2009). Enzymatic assembly of DNA molecules up to several hundred kilobases. Nat Methods 6, 343-345.

Goyal, A., Belardinelli, R., Maracci, C., Milon, P., and Rodnina, M.V. (2015). Directional transition from initiation to elongation in bacterial translation. Nucleic Acids Res 43, 10700 10712.

Goyal, A., Belardinelli, R., and Rodnina, M.V. (2017). Non-canonical Binding Site for Bacterial Initiation Factor 3 on the Large Ribosomal Subunit. Cell reports 20, 3113-3122. 
Greenfield, N.J. (2006a). Analysis of the kinetics of folding of proteins and peptides using circular dichroism. Nat Protoc 1, 2891-2899.

Greenfield, N.J. (2006b). Determination of the folding of proteins as a function of denaturants, osmolytes or ligands using circular dichroism. Nat Protoc 1, 2733-2741.

Greenfield, N.J. (2006c). Using circular dichroism spectra to estimate protein secondary structure. Nat Protoc 1, 2876-2890.

Gregersen, N., Bross, P., Vang, S., and Christensen, J.H. (2006). Protein misfolding and human disease. Annu Rev Genomics Hum Genet 7, 103-124.

Griffiths-Jones, S.R., Sharman, G.J., Maynard, A.J., and Searle, M.S. (1998). Modulation of intrinsic phi,psi propensities of amino acids by neighbouring residues in the coil regions of protein structures: NMR analysis and dissection of a beta-hairpin peptide. J Mol Biol 284, 15971609.

Gruebele, M. (2005). Downhill protein folding: evolution meets physics. C R Biol 328, 701-712. Gruebele, M., Dave, K., and Sukenik, S. (2016). Globular Protein Folding In Vitro and In Vivo. Annu Rev Biophys 45, 233-251.

Gutin, A.M., Abkevich, V.I., and Shakhnovich, E.I. (1995). Is burst hydrophobic collapse necessary for protein folding? Biochemistry 34, 3066-3076.

Hellen, C.U.T. (2018). Translation Termination and Ribosome Recycling in Eukaryotes. Cold Spring Harb Perspect Biol 10.

Holtkamp, W., Kokic, G., Jager, M., Mittelstaet, J., Komar, A.A., and Rodnina, M.V. (2015). Cotranslational protein folding on the ribosome monitored in real time. Science 350, 11041107.

Hu, W., Walters, B.T., Kan, Z.Y., Mayne, L., Rosen, L.E., Marqusee, S., and Englander, S.W. (2013). Stepwise protein folding at near amino acid resolution by hydrogen exchange and mass spectrometry. Proc Natl Acad Sci U S A 110, 7684-7689.

Huang, P.S., Boyken, S.E., and Baker, D. (2016). The coming of age of de novo protein design. Nature 537, 320-327.

Itzhaki, L.S., Otzen, D.E., and Fersht, A.R. (1995). The structure of the transition state for folding of chymotrypsin inhibitor 2 analysed by protein engineering methods: evidence for a nucleation-condensation mechanism for protein folding. J Mol Biol 254, 260-288.

Ivankov, D.N., and Finkelstein, A.V. (2020). Solution of Levinthal's Paradox and a Physical Theory of Protein Folding Times. Biomolecules 10.

Jain, A., Perisa, D., Fliedner, F., von Haeseler, A., and Ebersberger, I. (2019). The Evolutionary Traceability of a Protein. Genome Biol Evol 11, 531-545.

Jha, S.K., and Udgaonkar, J.B. (2010). Free energy barriers in protein folding and unfolding reactions. Current Science 99, 457-475. 
Johnson, K.A., Simpson, Z.B., and Blom, T. (2009a). FitSpace explorer: an algorithm to evaluate multidimensional parameter space in fitting kinetic data. Anal Biochem 387, 30-41. Johnson, K.A., Simpson, Z.B., and Blom, T. (2009b). Global kinetic explorer: a new computer program for dynamic simulation and fitting of kinetic data. Anal Biochem 387, 20-29.

Kaiser, C.M., Goldman, D.H., Chodera, J.D., Tinoco, I., Jr., and Bustamante, C. (2011). The ribosome modulates nascent protein folding. Science 334, 1723-1727.

Karplus, M., and Weaver, D.L. (1976). Protein-folding dynamics. Nature 260, 404-406.

Karplus, M., and Weaver, D.L. (1994). Protein folding dynamics: the diffusion-collision model and experimental data. Protein Sci 3, 650-668.

Kelkar, D.A., Khushoo, A., Yang, Z., and Skach, W.R. (2012). Kinetic analysis of ribosomebound fluorescent proteins reveals an early, stable, cotranslational folding intermediate. J Biol Chem 287, 2568-2578.

Kemp, G., Kudva, R., de la Rosa, A., and von Heijne, G. (2019). Force-Profile Analysis of the Cotranslational Folding of HemK and Filamin Domains: Comparison of Biochemical and Biophysical Folding Assays. J Mol Biol 431, 1308-1314.

Kim, P.S., and Baldwin, R.L. (1982). Specific intermediates in the folding reactions of small proteins and the mechanism of protein folding. Annu Rev Biochem 51, 459-489.

Klaholz, B.P. (2011). Molecular recognition and catalysis in translation termination complexes. Trends Biochem Sci 36, 282-292.

Komar, A.A. (2018). Unraveling co-translational protein folding: Concepts and methods. Methods 137, 71-81.

Kong, J., and Yu, S. (2007). Fourier transform infrared spectroscopic analysis of protein secondary structures. Acta Biochim Biophys Sin (Shanghai) 39, 549-559.

Korniy, N., Samatova, E., Anokhina, M.M., Peske, F., and Rodnina, M.V. (2019). Mechanisms and biomedical implications of -1 programmed ribosome frameshifting on viral and bacterial mRNAs. FEBS letters 593, 1468-1482.

Krichevsky, O., and Bonnet, G. (2002). Fluorescence correlation spectroscopy: the technique and its applications. Rep Prog Phys 65, 251-297.

Krishna, M.M., Hoang, L., Lin, Y., and Englander, S.W. (2004). Hydrogen exchange methods to study protein folding. Methods 34, 51-64.

Kuhlman, B., and Bradley, P. (2019). Advances in protein structure prediction and design. Nat Rev Mol Cell Biol 20, 681-697.

Lange, R., and Balny, C. (2002). UV-visible derivative spectroscopy under high pressure. Biochim Biophys Acta 1595, 80-93.

Leininger, S.E., Trovato, F., Nissley, D.A., and O'Brien, E.P. (2019). Domain topology, stability, and translation speed determine mechanical force generation on the ribosome. Proc Natl Acad Sci U S A 116, 5523-5532. 
Lenne, P.F., Raae, A.J., Altmann, S.M., Saraste, M., and Horber, J.K. (2000). States and transitions during forced unfolding of a single spectrin repeat. FEBS letters 476, 124-128.

Levinthal, C. (1969). How to Fold Graciously. Mossbauer Spectroscopy in Biological Systems Proceedings 67, 22-26.

Limpouchova, Z., and Prochazka, K. (2016). Theoretical principles of fluorescence spectroscopy. In Springer Ser Fluores, pp. 91-149.

Liu, K., Rehfus, J.E., Mattson, E., and Kaiser, C.M. (2017). The ribosome destabilizes native and non-native structures in a nascent multidomain protein. Protein Sci 26, 1439-1451.

Lu, J., and Deutsch, C. (2005). Folding zones inside the ribosomal exit tunnel. Nature structural \& molecular biology 12, 1123-1129.

Luitz, M.P., Barth, A., Crevenna, A.H., Bomblies, R., Lamb, D.C., and Zacharias, M. (2017). Covalent dye attachment influences the dynamics and conformational properties of flexible peptides. PLOS ONE 12, e0177139.

Lum, J.K., Neuweiler, H., and Fersht, A.R. (2012a). Long-range modulation of chain motions within the intrinsically disordered transactivation domain of tumor suppressor p53. J Am Chem Soc 134, 1617-1622.

Lum, J.K., Neuweiler, H., and Fersht, A.R. (2012b). Long-Range Modulation of Chain Motions within the Intrinsically Disordered Transactivation Domain of Tumor Suppressor p53. Journal of the American Chemical Society 134, 1617-1622.

Ma, B.G., Chen, L., Ji, H.F., Chen, Z.H., Yang, F.R., Wang, L., Qu, G., Jiang, Y.Y., Ji, C., and Zhang, H.Y. (2008). Characters of very ancient proteins. Biochem Biophys Res Commun 366, 607-611.

Marino, J., von Heijne, G., and Beckmann, R. (2016). Small protein domains fold inside the ribosome exit tunnel. FEBS letters 590, 655-660.

Marion, D. (2013). An introduction to biological NMR spectroscopy. Mol Cell Proteomics 12, 3006-3025.

Matouschek, A., Kellis, J.T., Jr., Serrano, L., and Fersht, A.R. (1989). Mapping the transition state and pathway of protein folding by protein engineering. Nature 340, 122-126.

Mercier, E., and Rodnina, M.V. (2018). Co-Translational Folding Trajectory of the HemK Helical Domain. Biochemistry 57, 3460-3464.

Merrick, W.C., and Pavitt, G.D. (2018). Protein Synthesis Initiation in Eukaryotic Cells. Cold Spring Harb Perspect Biol 10.

Meseth, U., Wohland, T., Rigler, R., and Vogel, H. (1999). Resolution of fluorescence correlation measurements. Biophysical journal 76, 1619-1631.

Michalet, X., Weiss, S., and Jager, M. (2006). Single-molecule fluorescence studies of protein folding and conformational dynamics. Chem Rev 106, 1785-1813. 
Milon, P., Konevega, A.L., Peske, F., Fabbretti, A., Gualerzi, C.O., and Rodnina, M.V. (2007). Transient kinetics, fluorescence, and FRET in studies of initiation of translation in bacteria. Methods Enzymol 430, 1-30.

Milon, P., and Rodnina, M. (2012). Kinetic control of translation initiation in bacteria. Critical Reviews in Biochemistry and Molecular Biology 47, 334-348.

Mittelstaet, J., Konevega, A.L., and Rodnina, M.V. (2013). A kinetic safety gate controlling the delivery of unnatural amino acids to the ribosome. Journal of the American Chemical Society 135, 17031-17038.

Morris, E.R., and Searle, M.S. (2012). Overview of protein folding mechanisms: experimental and theoretical approaches to probing energy landscapes. Curr Protoc Protein Sci Chapter 28, Unit 2822 21-22.

Nakatogawa, H., and Ito, K. (2002). The ribosomal exit tunnel functions as a discriminating gate. Cell 108, 629-636.

Nettels, D., Gopich, I.V., Hoffmann, A., and Schuler, B. (2007). Ultrafast dynamics of protein collapse from single-molecule photon statistics. Proceedings of the National Academy of Sciences of the United States of America 104, 2655-2660.

Neuman, K.C., and Nagy, A. (2008). Single-molecule force spectroscopy: optical tweezers, magnetic tweezers and atomic force microscopy. Nat Methods 5, 491-505.

Neuweiler, H., Banachewicz, W., and Fersht, A.R. (2010a). Kinetics of chain motions within a protein-folding intermediate. Proceedings of the National Academy of Sciences 107, 2210622110.

Neuweiler, H., Banachewicz, W., and Fersht, A.R. (2010b). Kinetics of chain motions within a protein-folding intermediate. Proc Natl Acad Sci U S A 107, 22106-22110.

Neuweiler, H., Johnson, C.M., and Fersht, A.R. (2009). Direct observation of ultrafast folding and denatured state dynamics in single protein molecules. Proc Natl Acad Sci U S A 106, 18569-18574.

Neuweiler, H., Schulz, A., Bohmer, M., Enderlein, J., and Sauer, M. (2003a). Measurement of submicrosecond intramolecular contact formation in peptides at the single-molecule level. $\mathrm{J}$ Am Chem Soc 125, 5324-5330.

Neuweiler, H., Schulz, A., Böhmer, M., Enderlein, J., and Sauer, M. (2003b). Measurement of Submicrosecond Intramolecular Contact Formation in Peptides at the Single-Molecule Level. Journal of the American Chemical Society 125, 5324-5330.

Nickson, A.A., and Clarke, J. (2010). What lessons can be learned from studying the folding of homologous proteins? Methods 52, 38-50.

Nilsson, O.B., Hedman, R., Marino, J., Wickles, S., Bischoff, L., Johansson, M., Muller-Lucks, A., Trovato, F., Puglisi, J.D., O'Brien, E.P., et al. (2015). Cotranslational Protein Folding inside the Ribosome Exit Tunnel. Cell reports 12, 1533-1540. 
Nissley, D.A., and O'Brien, E.P. (2018). Structural Origins of FRET-Observed Nascent Chain Compaction on the Ribosome. J Phys Chem B 122, 9927-9937.

Noe, F., Doose, S., Daidone, I., Lollmann, M., Sauer, M., Chodera, J.D., and Smith, J.C. (2011). Dynamical fingerprints for probing individual relaxation processes in biomolecular dynamics with simulations and kinetic experiments. Proc Natl Acad Sci U S A 108, 4822-4827. Noller, H.F., Ermolenko, D.N., Korostelev, A., Laurberg, M., Zhu, J., Asahara, H., Lancaster, L., Horan, L., Hirschi, A., Donohue, J.P., et al. (2011). Studies on the mechanisms of translocation and termination. In Ribosomes: Structure, Function, and Dynamics, M.V. Rodnina, W. Wintermeyer, and R. Green, eds. (Vienna: Springer Vienna), pp. 349-360.

Peske, F., Kuhlenkoetter, S., Rodnina, M.V., and Wintermeyer, W. (2014). Timing of GTP binding and hydrolysis by translation termination factor RF3. Nucleic Acids Res 42, 1812-1820. Peske, F., Rodnina, M.V., and Wintermeyer, W. (2005). Sequence of steps in ribosome recycling as defined by kinetic analysis. Mol Cell 18, 403-412.

Powers, E.T., Morimoto, R.I., Dillin, A., Kelly, J.W., and Balch, W.E. (2009). Biological and chemical approaches to diseases of proteostasis deficiency. Annu Rev Biochem 78, 959-991. Privalov, P.L. (1979). Stability of proteins: small globular proteins. Adv Protein Chem 33, 167241.

Rief, M., Gautel, M., Oesterhelt, F., Fernandez, J.M., and Gaub, H.E. (1997). Reversible unfolding of individual titin immunoglobulin domains by AFM. Science 276, 1109-1112.

Robson, B., and Pain, R.H. (1971). Analysis of the code relating sequence to conformation in proteins: possible implications for the mechanism of formation of helical regions. J Mol Biol 58, 237-259.

Rodnina, M.V. (2012). Quality control of mRNA decoding on the bacterial ribosome. Adv Protein Chem Struct Biol 86, 95-128.

Rodnina, M.V. (2013). The ribosome as a versatile catalyst: reactions at the peptidyl transferase center. Curr Opin Struct Biol 23, 595-602.

Rodnina, M.V. (2018). Translation in Prokaryotes. Cold Spring Harb Perspect Biol 10.

Rodnina, M.V., Beringer, M., and Wintermeyer, W. (2006). Mechanism of peptide bond formation on the ribosome. Q Rev Biophys 39, 203-225.

Rodnina, M.V., Fischer, N., Maracci, C., and Stark, H. (2017). Ribosome dynamics during decoding. Philos Trans R Soc Lond B Biol Sci 372.

Rodnina, M.V., and Wintermeyer, W. (1995). GTP consumption of elongation factor Tu during translation of heteropolymeric mRNAs. Proc Natl Acad Sci U S A 92, 1945-1949.

Rodnina, M.V., and Wintermeyer, W. (2001a). Fidelity of aminoacyl-tRNA selection on the ribosome: kinetic and structural mechanisms. Annu Rev Biochem 70, 415-435.

Rodnina, M.V., and Wintermeyer, W. (2001b). Ribosome fidelity: tRNA discrimination, proofreading and induced fit. Trends Biochem Sci 26, 124-130. 
Royer, C.A. (2006). Probing protein folding and conformational transitions with fluorescence. Chem Rev 106, 1769-1784.

Sadqi, M., Lapidus, L.J., and Munoz, V. (2003). How fast is protein hydrophobic collapse? Proc Natl Acad Sci U S A 100, 12117-12122.

Sali, A., Shakhnovich, E., and Karplus, M. (1994). How does a protein fold? Nature 369, 248251.

Samelson, A.J., Jensen, M.K., Soto, R.A., Cate, J.H., and Marqusee, S. (2016). Quantitative determination of ribosome nascent chain stability. Proc Natl Acad Sci U S A 113, 13402-13407. Santra, M., Dill, K.A., and de Graff, A.M.R. (2019). Proteostasis collapse is a driver of cell aging and death. Proc Natl Acad Sci U S A 116, 22173-22178.

Sauer, M., and Neuweiler, H. (2014). PET-FCS: probing rapid structural fluctuations of proteins and nucleic acids by single-molecule fluorescence quenching. Methods in molecular biology (Clifton, NJ) 1076, 597-615.

Saviotti, M.L., and Galley, W.C. (1974). Room temperature phosphorescence and the dynamic aspects of protein structure. Proc Natl Acad Sci U S A 71, 4154-4158.

Schagger, H. (2006). Tricine-SDS-PAGE. Nat Protoc 1, 16-22.

Scheraga, H.A., Khalili, M., and Liwo, A. (2007). Protein-folding dynamics: overview of molecular simulation techniques. Annu Rev Phys Chem 58, 57-83.

Schuler, B., and Hofmann, H. (2013). Single-molecule spectroscopy of protein folding dynamics--expanding scope and timescales. Curr Opin Struct Biol 23, 36-47.

Shakhnovich, E. (2006). Protein folding thermodynamics and dynamics: where physics, chemistry, and biology meet. Chem Rev 106, 1559-1588.

Shakhnovich, E.I., and Gutin, A.M. (1990). Implications of thermodynamics of protein folding for evolution of primary sequences. Nature 346, 773-775.

Sharma, A., Enderlein, J., and Kumbhakar, M. (2017). Photon Antibunching Reveals Static and Dynamic Quenching Interaction of Tryptophan with Atto-655. The journal of physical chemistry letters 8, 5821-5826.

Sharma, P.K., Xiang, Y., Kato, M., and Warshel, A. (2005). What are the roles of substrateassisted catalysis and proximity effects in peptide bond formation by the ribosome? Biochemistry 44, 11307-11314.

Simonetti, A., Marzi, S., Jenner, L., Myasnikov, A., Romby, P., Yusupova, G., Klaholz, B.P., and Yusupov, M. (2009). A structural view of translation initiation in bacteria. Cell Mol Life Sci 66, 423-436.

Sinha, K.K., and Udgaonkar, J.B. (2007). Dissecting the non-specific and specific components of the initial folding reaction of barstar by multi-site FRET measurements. J Mol Biol 370, 385405. 
Snow, C.D., Sorin, E.J., Rhee, Y.M., and Pande, V.S. (2005). How well can simulation predict protein folding kinetics and thermodynamics? Annu Rev Biophys Biomol Struct 34, 43-69.

Sridevi, K., Juneja, J., Bhuyan, A.K., Krishnamoorthy, G., and Udgaonkar, J.B. (2000). The slow folding reaction of barstar: the core tryptophan region attains tight packing before substantial secondary and tertiary structure formation and final compaction of the polypeptide chain. J Mol Biol 302, 479-495.

Stanley, N., Esteban-Martin, S., and De Fabritiis, G. (2014). Kinetic modulation of a disordered protein domain by phosphorylation. Nat Commun 5, 5272.

Swindells, M.B., MacArthur, M.W., and Thornton, J.M. (1995). Intrinsic phi, psi propensities of amino acids, derived from the coil regions of known structures. Nat Struct Biol 2, 596-603.

Taverna, D.M., and Goldstein, R.A. (2002). Why are proteins marginally stable? Proteins 46, 105-109.

Udgaonkar, J.B., and Baldwin, R.L. (1988). NMR evidence for an early framework intermediate on the folding pathway of ribonuclease A. Nature 335, 694-699.

Varela, A.E., England, K.A., and Cavagnero, S. (2019). Kinetic trapping in protein folding. Protein Eng Des Sel.

Waldauer, S.A., Bakajin, O., and Lapidus, L.J. (2010). Extremely slow intramolecular diffusion in unfolded protein L. Proceedings of the National Academy of Sciences of the United States of America 107, 13713-13717.

Wallin, G., and Aqvist, J. (2010). The transition state for peptide bond formation reveals the ribosome as a water trap. Proc Natl Acad Sci U S A 107, 1888-1893.

Walters, B.T., Mayne, L., Hinshaw, J.R., Sosnick, T.R., and Englander, S.W. (2013). Folding of a large protein at high structural resolution. Proc Natl Acad Sci U S A 110, 18898-18903.

Wetlaufer, D.B. (1973). Nucleation, rapid folding, and globular intrachain regions in proteins. Proc Natl Acad Sci U S A 70, 697-701.

Yang, G., Cecconi, C., Baase, W.A., Vetter, I.R., Breyer, W.A., Haack, J.A., Matthews, B.W., Dahlquist, F.W., and Bustamante, C. (2000). Solid-state synthesis and mechanical unfolding of polymers of T4 lysozyme. Proc Natl Acad Sci U S A 97, 139-144.

Yang, Z., Shipman, L., Zhang, M., Anton, B.P., Roberts, R.J., and Cheng, X. (2004). Structural characterization and comparative phylogenetic analysis of Escherichia coli HemK, a protein (N5)-glutamine methyltransferase. Journal of molecular biology 340, 695-706.

Zhou, J., Korostelev, A., Lancaster, L., and Noller, H.F. (2012). Crystal structures of 70 S ribosomes bound to release factors RF1, RF2 and RF3. Curr Opin Struct Biol 22, 733-742.

Zhuang, X., Ha, T., Kim, H.D., Centner, T., Labeit, S., and Chu, S. (2000). Fluorescence quenching: A tool for single-molecule protein-folding study. Proc Natl Acad Sci U S A 97, 14241-14244. 


\section{Appendix}

\section{List of Figures}

Figure 1 Ribosome structure .11

Figure 2 Translation Initiation .12

Figure 3 Elongation cycle .14

Figure 4 Release and Recycling .16

Figure 5 Hierarchy of protein structure .17

Figure 6 Protein folding models .19

Figure 7 Amino acid hydrophobicity .20

Figure 8 Folding energy landscape. 21

Figure 9 Fluorescence correlation spectroscopy... .25

Figure 10 Co-translational folding of HemK NTD revealed by high-resolution FPA . .45

Figure 11 Representative SDS PAGE of FPA for HemK 4xA variant. .48

Figure 12 Monitoring dynamics of HemK constructs in solution by PET-FCS .49

Figure 13 Dynamics of HemK on the ribosome monitored by PET-FCS .51

Figure 14 FPA of HemK W6F .52

Figure 15 ATTO 655 triplet state in RNC . .53

Figure 16 Kinetic model 1 of conformational dynamics of HemK RNCs .56

Figure 17 Kinetic model 2 of conformational dynamics of HemK RNCs. .57

Figure 18 Kinetic model 3 of conformational dynamics of HemK RNCs .58

Figure 19 Global fitting of HemK W6F RNC ACFs with increasing free Trp concentration ....59

Figure 20 Rates of the conformational dynamics of HemK RNCs .60

Figure 21 Free energy barriers between different chain conformations 62 


\section{List of Tables}

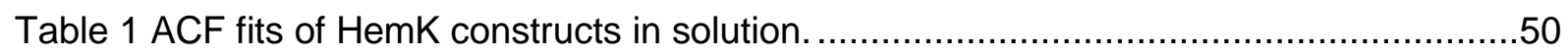

Table 2 Results of analytical fits of PET-FCS ACF for RNCs...........................................

Table 3 Results of global fitting of the free Trp titration dataset .......................................59

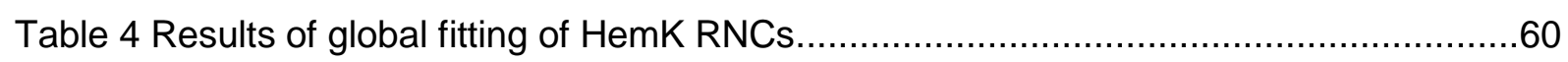

Table 5 Free energy calculations for HemK RNC constructs .........................................62

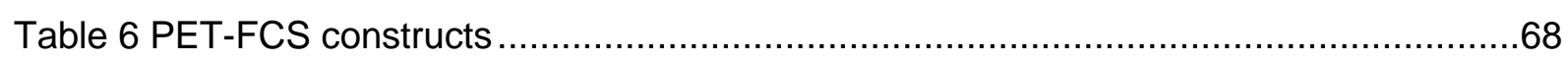

Table 7 Force profile constructs of full-length wt HemK .............................................69

Table 8 Upper and lower boundaries of the kinetic fit ............................................... 74

\section{List of Equations}

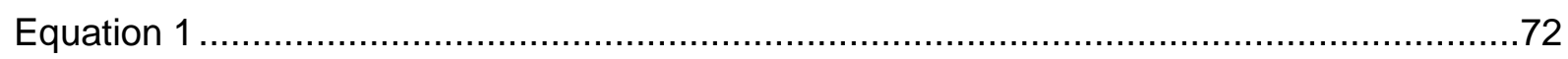

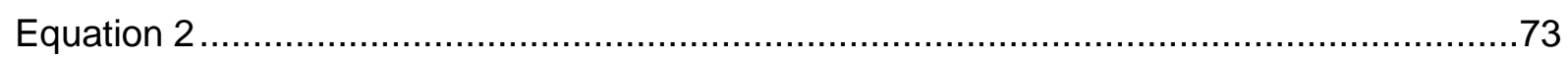




\section{DNA sequence 5' to 3' of HemK wt FPA constructs.}

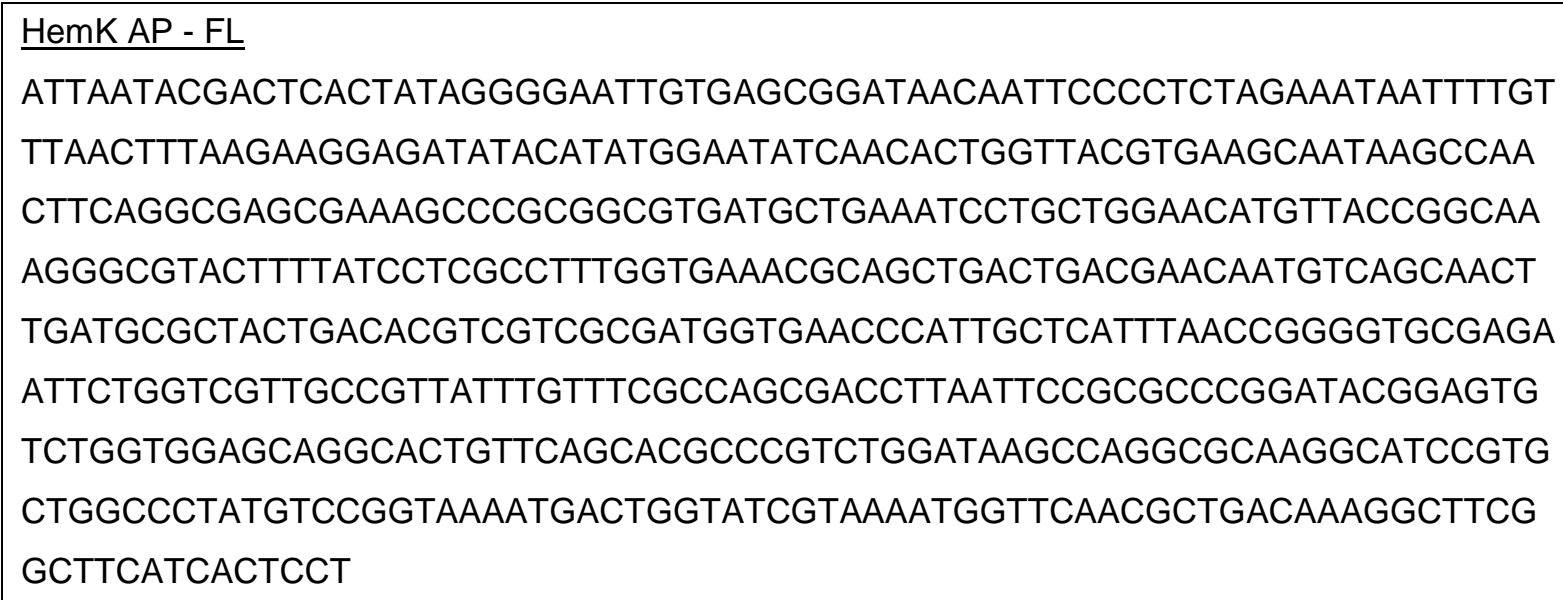

ATTAATACGACTCACTATAGGGGAATTGTGAGCGGATAACAATTCCCCTCTAGAAATAATTTTGT TTAACTTTAAGAAGGAGATATACATATGGAATATCAACACTGGTTACGTGAAGCAATAAGCCAA CTTCAGGCGAGCGAAAGCCCGCGGCGTGATGCTGAAATCCTGCTGGAACATGTTACCGGCAA AGGGCGTACTTTTATCCTCGCCTTTGGTGAAACGCAGCTGACTGACGAACAATGTCAGCAACT TGATGCGCTACTGACACGTCGTCGCGATGGTGAACCCATTGCTCATTTAACCGGGGTGCGAGA ATTCTGGTCGTTGCCGTTATTTGTTTCGCCAGCGACCTTAATTCCGCGCCCGGATACGGAGTG TCTGGTGGAGCAGTTCAGCACGCCCGTCTGGATAAGCCAGGCGCAAGGCATCCGTGCTGGCC CTATGTCCGGTAAAATGACTGGTATCGTAAAATGGTTCAACGCTGACAAAGGCTTCGGCTTCAT CACTCCT

HemK AP - 99

ATTAATACGACTCACTATAGGGGAATTGTGAGCGGATAACAATTCCCCTCTAGAAATAATTTTGT TTAACTTTAAGAAGGAGATATACATATGGAATATCAACACTGGTTACGTGAAGCAATAAGCCAA CTTCAGGCGAGCGAAAGCCCGCGGCGTGATGCTGAAATCCTGCTGGAACATGTTACCGGCAA AGGGCGTACTTTTATCCTCGCCTTTGGTGAAACGCAGCTGACTGACGAACAATGTCAGCAACT TGATGCGCTACTGACACGTCGTCGCGATGGTGAACCCATTGCTCATTTAACCGGGGTGCGAGA ATTCTGGTCGTTGCCGTTATTTGTTTCGCCAGCGACCTTAATTCCGCGCCCGGATACGGAGTG TCTGGTGTTCAGCACGCCCGTCTGGATAAGCCAGGCGCAAGGCATCCGTGCTGGCCCTATGT CCGGTAAAATGACTGGTATCGTAAAATGGTTCAACGCTGACAAAGGCTTCGGCTTCATCACTCC $\mathrm{T}$

HemK AP -97

ATTAATACGACTCACTATAGGGGAATTGTGAGCGGATAACAATTCCCCTCTAGAAATAATTTTGT TTAACTTTAAGAAGGAGATATACATATGGAATATCAACACTGGTTACGTGAAGCAATAAGCCAA CTTCAGGCGAGCGAAAGCCCGCGGCGTGATGCTGAAATCCTGCTGGAACATGTTACCGGCAA 
AGGGCGTACTTTTATCCTCGCCTTTGGTGAAACGCAGCTGACTGACGAACAATGTCAGCAACT TGATGCGCTACTGACACGTCGTCGCGATGGTGAACCCATTGCTCATTTAACCGGGGTGCGAGA ATTCTGGTCGTTGCCGTTATTTGTTTCGCCAGCGACCTTAATTCCGCGCCCGGATACGGAGTG TTTCAGCACGCCCGTCTGGATAAGCCAGGCGCAAGGCATCCGTGCTGGCCCTATGTCCGGTA AAATGACTGGTATCGTAAAATGGTTCAACGCTGACAAAGGCTTCGGCTTCATCACTCCT

HemK AP - 95

ATTAATACGACTCACTATAGGGGAATTGTGAGCGGATAACAATTCCCCTCTAGAAATAATTTTGT TTAACTTTAAGAAGGAGATATACATATGGAATATCAACACTGGTTACGTGAAGCAATAAGCCAA CTTCAGGCGAGCGAAAGCCCGCGGCGTGATGCTGAAATCCTGCTGGAACATGTTACCGGCAA AGGGCGTACTTTTATCCTCGCCTTTGGTGAAACGCAGCTGACTGACGAACAATGTCAGCAACT TGATGCGCTACTGACACGTCGTCGCGATGGTGAACCCATTGCTCATTTAACCGGGGTGCGAGA ATTCTGGTCGTTGCCGTTATTTGTTTCGCCAGCGACCTTAATTCCGCGCCCGGATACGTTCAGC ACGCCCGTCTGGATAAGCCAGGCGCAAGGCATCCGTGCTGGCCCTATGTCCGGTAAAATGAC TGGTATCGTAAAATGGTTCAACGCTGACAAAGGCTTCGGCTTCATCACTCCT

HemK AP - 93

ATTAATACGACTCACTATAGGGGAATTGTGAGCGGATAACAATTCCCCTCTAGAAATAATTTTGT TTAACTTTAAGAAGGAGATATACATATGGAATATCAACACTGGTTACGTGAAGCAATAAGCCAA CTTCAGGCGAGCGAAAGCCCGCGGCGTGATGCTGAAATCCTGCTGGAACATGTTACCGGCAA AGGGCGTACTTTTATCCTCGCCTTTGGTGAAACGCAGCTGACTGACGAACAATGTCAGCAACT TGATGCGCTACTGACACGTCGTCGCGATGGTGAACCCATTGCTCATTTAACCGGGGTGCGAGA ATTCTGGTCGTTGCCGTTATTTGTTTCGCCAGCGACCTTAATTCCGCGCCCGTTCAGCACGCC CGTCTGGATAAGCCAGGCGCAAGGCATCCGTGCTGGCCCTATGTCCGGTAAAATGACTGGTAT CGTAAAATGGTTCAACGCTGACAAAGGCTTCGGCTTCATCACTCCT

HemK AP - 91

ATTAATACGACTCACTATAGGGGAATTGTGAGCGGATAACAATTCCCCTCTAGAAATAATTTTGT TTAACTTTAAGAAGGAGATATACATATGGAATATCAACACTGGTTACGTGAAGCAATAAGCCAA CTTCAGGCGAGCGAAAGCCCGCGGCGTGATGCTGAAATCCTGCTGGAACATGTTACCGGCAA AGGGCGTACTTTTATCCTCGCCTTTGGTGAAACGCAGCTGACTGACGAACAATGTCAGCAACT TGATGCGCTACTGACACGTCGTCGCGATGGTGAACCCATTGCTCATTTAACCGGGGTGCGAGA ATTCTGGTCGTTGCCGTTATTTGTTTCGCCAGCGACCTTAATTCCGTTCAGCACGCCCGTCTGG ATAAGCCAGGCGCAAGGCATCCGTGCTGGCCCTATGTCCGGTAAAATGACTGGTATCGTAAAA TGGTTCAACGCTGACAAAGGCTTCGGCTTCATCACTCCT

HemK AP - 90

ATTAATACGACTCACTATAGGGGAATTGTGAGCGGATAACAATTCCCCTCTAGAAATAATTTTGT TTAACTTTAAGAAGGAGATATACATATGGAATATCAACACTGGTTACGTGAAGCAATAAGCCAA 
CTTCAGGCGAGCGAAAGCCCGCGGCGTGATGCTGAAATCCTGCTGGAACATGTTACCGGCAA AGGGCGTACTTTTATCCTCGCCTTTGGTGAAACGCAGCTGACTGACGAACAATGTCAGCAACT TGATGCGCTACTGACACGTCGTCGCGATGGTGAACCCATTGCTCATTTAACCGGGGTGCGAGA ATTCTGGTCGTTGCCGTTATTTGTTTCGCCAGCGACCTTAATTTTCAGCACGCCCGTCTGGATA AGCCAGGCGCAAGGCATCCGTGCTGGCCCTATGTCCGGTAAAATGACTGGTATCGTAAAATGG TTCAACGCTGACAAAGGCTTCGGCTTCATCACTCCT

$\underline{\text { HemK AP }-89}$

ATTAATACGACTCACTATAGGGGAATTGTGAGCGGATAACAATTCCCCTCTAGAAATAATTTTGT TTAACTTTAAGAAGGAGATATACATATGGAATATCAACACTGGTTACGTGAAGCAATAAGCCAA CTTCAGGCGAGCGAAAGCCCGCGGCGTGATGCTGAAATCCTGCTGGAACATGTTACCGGCAA AGGGCGTACTTTTATCCTCGCCTTTGGTGAAACGCAGCTGACTGACGAACAATGTCAGCAACT TGATGCGCTACTGACACGTCGTCGCGATGGTGAACCCATTGCTCATTTAACCGGGGTGCGAGA ATTCTGGTCGTTGCCGTTATTTGTTTCGCCAGCGACCTTATTCAGCACGCCCGTCTGGATAAGC CAGGCGCAAGGCATCCGTGCTGGCCCTATGTCCGGTAAAATGACTGGTATCGTAAAATGGTTC AACGCTGACAAAGGCTTCGGCTTCATCACTCCT

HemK AP -88

ATTAATACGACTCACTATAGGGGAATTGTGAGCGGATAACAATTCCCCTCTAGAAATAATTTTGT TTAACTTTAAGAAGGAGATATACATATGGAATATCAACACTGGTTACGTGAAGCAATAAGCCAA CTTCAGGCGAGCGAAAGCCCGCGGCGTGATGCTGAAATCCTGCTGGAACATGTTACCGGCAA AGGGCGTACTTTTATCCTCGCCTTTGGTGAAACGCAGCTGACTGACGAACAATGTCAGCAACT TGATGCGCTACTGACACGTCGTCGCGATGGTGAACCCATTGCTCATTTAACCGGGGTGCGAGA ATTCTGGTCGTTGCCGTTATTTGTTTCGCCAGCGACCTTCAGCACGCCCGTCTGGATAAGCCA GGCGCAAGGCATCCGTGCTGGCCCTATGTCCGGTAAAATGACTGGTATCGTAAAATGGTTCAA CGCTGACAAAGGCTTCGGCTTCATCACTCCT

HemK AP - 87

ATTAATACGACTCACTATAGGGGAATTGTGAGCGGATAACAATTCCCCTCTAGAAATAATTTTGT TTAACTTTAAGAAGGAGATATACATATGGAATATCAACACTGGTTACGTGAAGCAATAAGCCAA CTTCAGGCGAGCGAAAGCCCGCGGCGTGATGCTGAAATCCTGCTGGAACATGTTACCGGCAA AGGGCGTACTTTTATCCTCGCCTTTGGTGAAACGCAGCTGACTGACGAACAATGTCAGCAACT TGATGCGCTACTGACACGTCGTCGCGATGGTGAACCCATTGCTCATTTAACCGGGGTGCGAGA ATTCTGGTCGTTGCCGTTATTTGTTTCGCCAGCGTTCAGCACGCCCGTCTGGATAAGCCAGGC GCAAGGCATCCGTGCTGGCCCTATGTCCGGTAAAATGACTGGTATCGTAAAATGGTTCAACGC TGACAAAGGCTTCGGCTTCATCACTCCT 
HemK AP - 86

ATTAATACGACTCACTATAGGGGAATTGTGAGCGGATAACAATTCCCCTCTAGAAATAATTTTGT TTAACTTTAAGAAGGAGATATACATATGGAATATCAACACTGGTTACGTGAAGCAATAAGCCAA CTTCAGGCGAGCGAAAGCCCGCGGCGTGATGCTGAAATCCTGCTGGAACATGTTACCGGCAA AGGGCGTACTTTTATCCTCGCCTTTGGTGAAACGCAGCTGACTGACGAACAATGTCAGCAACT TGATGCGCTACTGACACGTCGTCGCGATGGTGAACCCATTGCTCATTTAACCGGGGTGCGAGA ATTCTGGTCGTTGCCGTTATTTGTTTCGCCATTCAGCACGCCCGTCTGGATAAGCCAGGCGCA AGGCATCCGTGCTGGCCCTATGTCCGGTAAAATGACTGGTATCGTAAAATGGTTCAACGCTGA CAAAGGCTTCGGCTTCATCACTCCT

HemK AP - 85

ATTAATACGACTCACTATAGGGGAATTGTGAGCGGATAACAATTCCCCTCTAGAAATAATTTTGT TTAACTTTAAGAAGGAGATATACATATGGAATATCAACACTGGTTACGTGAAGCAATAAGCCAA CTTCAGGCGAGCGAAAGCCCGCGGCGTGATGCTGAAATCCTGCTGGAACATGTTACCGGCAA AGGGCGTACTTTTATCCTCGCCTTTGGTGAAACGCAGCTGACTGACGAACAATGTCAGCAACT TGATGCGCTACTGACACGTCGTCGCGATGGTGAACCCATTGCTCATTTAACCGGGGTGCGAGA ATTCTGGTCGTTGCCGTTATTTGTTTCGTTCAGCACGCCCGTCTGGATAAGCCAGGCGCAAGG CATCCGTGCTGGCCCTATGTCCGGTAAAATGACTGGTATCGTAAAATGGTTCAACGCTGACAA AGGCTTCGGCTTCATCACTCCT

HemK AP - 84

ATTAATACGACTCACTATAGGGGAATTGTGAGCGGATAACAATTCCCCTCTAGAAATAATTTTGT TTAACTTTAAGAAGGAGATATACATATGGAATATCAACACTGGTTACGTGAAGCAATAAGCCAA CTTCAGGCGAGCGAAAGCCCGCGGCGTGATGCTGAAATCCTGCTGGAACATGTTACCGGCAA AGGGCGTACTTTTATCCTCGCCTTTGGTGAAACGCAGCTGACTGACGAACAATGTCAGCAACT TGATGCGCTACTGACACGTCGTCGCGATGGTGAACCCATTGCTCATTTAACCGGGGTGCGAGA ATTCTGGTCGTTGCCGTTATTTGTTTTCAGCACGCCCGTCTGGATAAGCCAGGCGCAAGGCAT CCGTGCTGGCCCTATGTCCGGTAAAATGACTGGTATCGTAAAATGGTTCAACGCTGACAAAGG CTTCGGCTTCATCACTCCT

HemK AP - 83

ATTAATACGACTCACTATAGGGGAATTGTGAGCGGATAACAATTCCCCTCTAGAAATAATTTTGT TTAACTTTAAGAAGGAGATATACATATGGAATATCAACACTGGTTACGTGAAGCAATAAGCCAA CTTCAGGCGAGCGAAAGCCCGCGGCGTGATGCTGAAATCCTGCTGGAACATGTTACCGGCAA AGGGCGTACTTTTATCCTCGCCTTTGGTGAAACGCAGCTGACTGACGAACAATGTCAGCAACT TGATGCGCTACTGACACGTCGTCGCGATGGTGAACCCATTGCTCATTTAACCGGGGTGCGAGA ATTCTGGTCGTTGCCGTTATTTTTCAGCACGCCCGTCTGGATAAGCCAGGCGCAAGGCATCCG 
TGCTGGCCCTATGTCCGGTAAAATGACTGGTATCGTAAAATGGTTCAACGCTGACAAAGGCTT CGGCTTCATCACTCCT

HemK AP - 82

ATTAATACGACTCACTATAGGGGAATTGTGAGCGGATAACAATTCCCCTCTAGAAATAATTTTGT TTAACTTTAAGAAGGAGATATACATATGGAATATCAACACTGGTTACGTGAAGCAATAAGCCAA CTTCAGGCGAGCGAAAGCCCGCGGCGTGATGCTGAAATCCTGCTGGAACATGTTACCGGCAA AGGGCGTACTTTTATCCTCGCCTTTGGTGAAACGCAGCTGACTGACGAACAATGTCAGCAACT TGATGCGCTACTGACACGTCGTCGCGATGGTGAACCCATTGCTCATTTAACCGGGGTGCGAGA ATTCTGGTCGTTGCCGTTATTCAGCACGCCCGTCTGGATAAGCCAGGCGCAAGGCATCCGTGC TGGCCCTATGTCCGGTAAAATGACTGGTATCGTAAAATGGTTCAACGCTGACAAAGGCTTCGG CTTCATCACTCCT

HemK AP - 81

ATTAATACGACTCACTATAGGGGAATTGTGAGCGGATAACAATTCCCCTCTAGAAATAATTTTGT TTAACTTTAAGAAGGAGATATACATATGGAATATCAACACTGGTTACGTGAAGCAATAAGCCAA CTTCAGGCGAGCGAAAGCCCGCGGCGTGATGCTGAAATCCTGCTGGAACATGTTACCGGCAA AGGGCGTACTTTTATCCTCGCCTTTGGTGAAACGCAGCTGACTGACGAACAATGTCAGCAACT TGATGCGCTACTGACACGTCGTCGCGATGGTGAACCCATTGCTCATTTAACCGGGGTGCGAGA ATTCTGGTCGTTGCCGTTCAGCACGCCCGTCTGGATAAGCCAGGCGCAAGGCATCCGTGCTG GCCCTATGTCCGGTAAAATGACTGGTATCGTAAAATGGTTCAACGCTGACAAAGGCTTCGGCT TCATCACTCCT

HemK AP -80

ATTAATACGACTCACTATAGGGGAATTGTGAGCGGATAACAATTCCCCTCTAGAAATAATTTTGT TTAACTTTAAGAAGGAGATATACATATGGAATATCAACACTGGTTACGTGAAGCAATAAGCCAA CTTCAGGCGAGCGAAAGCCCGCGGCGTGATGCTGAAATCCTGCTGGAACATGTTACCGGCAA AGGGCGTACTTTTATCCTCGCCTTTGGTGAAACGCAGCTGACTGACGAACAATGTCAGCAACT TGATGCGCTACTGACACGTCGTCGCGATGGTGAACCCATTGCTCATTTAACCGGGGTGCGAGA ATTCTGGTCGTTGTTCAGCACGCCCGTCTGGATAAGCCAGGCGCAAGGCATCCGTGCTGGCC CTATGTCCGGTAAAATGACTGGTATCGTAAAATGGTTCAACGCTGACAAAGGCTTCGGCTTCAT CACTCCT

HemK AP - 79

ATTAATACGACTCACTATAGGGGAATTGTGAGCGGATAACAATTCCCCTCTAGAAATAATTTTGT TTAACTTTAAGAAGGAGATATACATATGGAATATCAACACTGGTTACGTGAAGCAATAAGCCAA CTTCAGGCGAGCGAAAGCCCGCGGCGTGATGCTGAAATCCTGCTGGAACATGTTACCGGCAA AGGGCGTACTTTTATCCTCGCCTTTGGTGAAACGCAGCTGACTGACGAACAATGTCAGCAACT TGATGCGCTACTGACACGTCGTCGCGATGGTGAACCCATTGCTCATTTAACCGGGGTGCGAGA 


\begin{abstract}
ATTCTGGTCGTTCAGCACGCCCGTCTGGATAAGCCAGGCGCAAGGCATCCGTGCTGGCCCTA TGTCCGGTAAAATGACTGGTATCGTAAAATGGTTCAACGCTGACAAAGGCTTCGGCTTCATCAC TCCT

HemK AP - 78

ATTAATACGACTCACTATAGGGGAATTGTGAGCGGATAACAATTCCCCTCTAGAAATAATTTTGT TTAACTTTAAGAAGGAGATATACATATGGAATATCAACACTGGTTACGTGAAGCAATAAGCCAA CTTCAGGCGAGCGAAAGCCCGCGGCGTGATGCTGAAATCCTGCTGGAACATGTTACCGGCAA AGGGCGTACTTTTATCCTCGCCTTTGGTGAAACGCAGCTGACTGACGAACAATGTCAGCAACT TGATGCGCTACTGACACGTCGTCGCGATGGTGAACCCATTGCTCATTTAACCGGGGTGCGAGA ATTCTGGTTCAGCACGCCCGTCTGGATAAGCCAGGCGCAAGGCATCCGTGCTGGCCCTATGT CCGGTAAAATGACTGGTATCGTAAAATGGTTCAACGCTGACAAAGGCTTCGGCTTCATCACTCC $\mathrm{T}$

HemK AP -77

ATTAATACGACTCACTATAGGGGAATTGTGAGCGGATAACAATTCCCCTCTAGAAATAATTTTGT TTAACTTTAAGAAGGAGATATACATATGGAATATCAACACTGGTTACGTGAAGCAATAAGCCAA CTTCAGGCGAGCGAAAGCCCGCGGCGTGATGCTGAAATCCTGCTGGAACATGTTACCGGCAA AGGGCGTACTTTTATCCTCGCCTTTGGTGAAACGCAGCTGACTGACGAACAATGTCAGCAACT TGATGCGCTACTGACACGTCGTCGCGATGGTGAACCCATTGCTCATTTAACCGGGGTGCGAGA ATTCTTCAGCACGCCCGTCTGGATAAGCCAGGCGCAAGGCATCCGTGCTGGCCCTATGTCCG GTAAAATGACTGGTATCGTAAAATGGTTCAACGCTGACAAAGGCTTCGGCTTCATCACTCCT

HemK AP - 76

ATTAATACGACTCACTATAGGGGAATTGTGAGCGGATAACAATTCCCCTCTAGAAATAATTTTGT TTAACTTTAAGAAGGAGATATACATATGGAATATCAACACTGGTTACGTGAAGCAATAAGCCAA CTTCAGGCGAGCGAAAGCCCGCGGCGTGATGCTGAAATCCTGCTGGAACATGTTACCGGCAA AGGGCGTACTTTTATCCTCGCCTTTGGTGAAACGCAGCTGACTGACGAACAATGTCAGCAACT TGATGCGCTACTGACACGTCGTCGCGATGGTGAACCCATTGCTCATTTAACCGGGGTGCGAGA ATTCAGCACGCCCGTCTGGATAAGCCAGGCGCAAGGCATCCGTGCTGGCCCTATGTCCGGTA AAATGACTGGTATCGTAAAATGGTTCAACGCTGACAAAGGCTTCGGCTTCATCACTCCT

HemK AP -75

ATTAATACGACTCACTATAGGGGAATTGTGAGCGGATAACAATTCCCCTCTAGAAATAATTTTGT TTAACTTTAAGAAGGAGATATACATATGGAATATCAACACTGGTTACGTGAAGCAATAAGCCAA CTTCAGGCGAGCGAAAGCCCGCGGCGTGATGCTGAAATCCTGCTGGAACATGTTACCGGCAA AGGGCGTACTTTTATCCTCGCCTTTGGTGAAACGCAGCTGACTGACGAACAATGTCAGCAACT TGATGCGCTACTGACACGTCGTCGCGATGGTGAACCCATTGCTCATTTAACCGGGGTGCGATT
\end{abstract}


CAGCACGCCCGTCTGGATAAGCCAGGCGCAAGGCATCCGTGCTGGCCCTATGTCCGGTAAAA TGACTGGTATCGTAAAATGGTTCAACGCTGACAAAGGCTTCGGCTTCATCACTCCT

HemK AP - 73

ATTAATACGACTCACTATAGGGGAATTGTGAGCGGATAACAATTCCCCTCTAGAAATAATTTTGT TTAACTTTAAGAAGGAGATATACATATGGAATATCAACACTGGTTACGTGAAGCAATAAGCCAA CTTCAGGCGAGCGAAAGCCCGCGGCGTGATGCTGAAATCCTGCTGGAACATGTTACCGGCAA AGGGCGTACTTTTATCCTCGCCTTTGGTGAAACGCAGCTGACTGACGAACAATGTCAGCAACT TGATGCGCTACTGACACGTCGTCGCGATGGTGAACCCATTGCTCATTTAACCGGGTTCAGCAC GCCCGTCTGGATAAGCCAGGCGCAAGGCATCCGTGCTGGCCCTATGTCCGGTAAAATGACTG GTATCGTAAAATGGTTCAACGCTGACAAAGGCTTCGGCTTCATCACTCCT

HemK AP - 71

ATTAATACGACTCACTATAGGGGAATTGTGAGCGGATAACAATTCCCCTCTAGAAATAATTTTGT TTAACTTTAAGAAGGAGATATACATATGGAATATCAACACTGGTTACGTGAAGCAATAAGCCAA CTTCAGGCGAGCGAAAGCCCGCGGCGTGATGCTGAAATCCTGCTGGAACATGTTACCGGCAA AGGGCGTACTTTTATCCTCGCCTTTGGTGAAACGCAGCTGACTGACGAACAATGTCAGCAACT TGATGCGCTACTGACACGTCGTCGCGATGGTGAACCCATTGCTCATTTATTCAGCACGCCCGT CTGGATAAGCCAGGCGCAAGGCATCCGTGCTGGCCCTATGTCCGGTAAAATGACTGGTATCGT AAAATGGTTCAACGCTGACAAAGGCTTCGGCTTCATCACTCCT

HemK AP - 70

ATTAATACGACTCACTATAGGGGAATTGTGAGCGGATAACAATTCCCCTCTAGAAATAATTTTGT TTAACTTTAAGAAGGAGATATACATATGGAATATCAACACTGGTTACGTGAAGCAATAAGCCAA CTTCAGGCGAGCGAAAGCCCGCGGCGTGATGCTGAAATCCTGCTGGAACATGTTACCGGCAA AGGGCGTACTTTTATCCTCGCCTTTGGTGAAACGCAGCTGACTGACGAACAATGTCAGCAACT TGATGCGCTACTGACACGTCGTCGCGATGGTGAACCCATTGCTCATTTCAGCACGCCCGTCTG GATAAGCCAGGCGCAAGGCATCCGTGCTGGCCCTATGTCCGGTAAAATGACTGGTATCGTAAA ATGGTTCAACGCTGACAAAGGCTTCGGCTTCATCACTCCT

HemK AP -68

ATTAATACGACTCACTATAGGGGAATTGTGAGCGGATAACAATTCCCCTCTAGAAATAATTTTGT TTAACTTTAAGAAGGAGATATACATATGGAATATCAACACTGGTTACGTGAAGCAATAAGCCAA CTTCAGGCGAGCGAAAGCCCGCGGCGTGATGCTGAAATCCTGCTGGAACATGTTACCGGCAA AGGGCGTACTTTTATCCTCGCCTTTGGTGAAACGCAGCTGACTGACGAACAATGTCAGCAACT TGATGCGCTACTGACACGTCGTCGCGATGGTGAACCCATTTTCAGCACGCCCGTCTGGATAAG 
CCAGGCGCAAGGCATCCGTGCTGGCCCTATGTCCGGTAAAATGACTGGTATCGTAAAATGGTT CAACGCTGACAAAGGCTTCGGCTTCATCACTCCT

HemK AP -66

ATTAATACGACTCACTATAGGGGAATTGTGAGCGGATAACAATTCCCCTCTAGAAATAATTTTGT TTAACTTTAAGAAGGAGATATACATATGGAATATCAACACTGGTTACGTGAAGCAATAAGCCAA CTTCAGGCGAGCGAAAGCCCGCGGCGTGATGCTGAAATCCTGCTGGAACATGTTACCGGCAA AGGGCGTACTTTTATCCTCGCCTTTGGTGAAACGCAGCTGACTGACGAACAATGTCAGCAACT TGATGCGCTACTGACACGTCGTCGCGATGGTGAATTCAGCACGCCCGTCTGGATAAGCCAGG CGCAAGGCATCCGTGCTGGCCCTATGTCCGGTAAAATGACTGGTATCGTAAAATGGTTCAACG CTGACAAAGGCTTCGGCTTCATCACTCCT

HemK AP - 64

ATTAATACGACTCACTATAGGGGAATTGTGAGCGGATAACAATTCCCCTCTAGAAATAATTTTGT TTAACTTTAAGAAGGAGATATACATATGGAATATCAACACTGGTTACGTGAAGCAATAAGCCAA CTTCAGGCGAGCGAAAGCCCGCGGCGTGATGCTGAAATCCTGCTGGAACATGTTACCGGCAA AGGGCGTACTTTTATCCTCGCCTTTGGTGAAACGCAGCTGACTGACGAACAATGTCAGCAACT TGATGCGCTACTGACACGTCGTCGCGATTTCAGCACGCCCGTCTGGATAAGCCAGGCGCAAG GCATCCGTGCTGGCCCTATGTCCGGTAAAATGACTGGTATCGTAAAATGGTTCAACGCTGACA AAGGCTTCGGCTTCATCACTCCT

HemK AP - 62

ATTAATACGACTCACTATAGGGGAATTGTGAGCGGATAACAATTCCCCTCTAGAAATAATTTTGT TTAACTTTAAGAAGGAGATATACATATGGAATATCAACACTGGTTACGTGAAGCAATAAGCCAA CTTCAGGCGAGCGAAAGCCCGCGGCGTGATGCTGAAATCCTGCTGGAACATGTTACCGGCAA AGGGCGTACTTTTATCCTCGCCTTTGGTGAAACGCAGCTGACTGACGAACAATGTCAGCAACT TGATGCGCTACTGACACGTCGTTTCAGCACGCCCGTCTGGATAAGCCAGGCGCAAGGCATCC GTGCTGGCCCTATGTCCGGTAAAATGACTGGTATCGTAAAATGGTTCAACGCTGACAAAGGCT TCGGCTTCATCACTCCT

HemK AP - 58

ATTAATACGACTCACTATAGGGGAATTGTGAGCGGATAACAATTCCCCTCTAGAAATAATTTTGT TTAACTTTAAGAAGGAGATATACATATGGAATATCAACACTGGTTACGTGAAGCAATAAGCCAA CTTCAGGCGAGCGAAAGCCCGCGGCGTGATGCTGAAATCCTGCTGGAACATGTTACCGGCAA AGGGCGTACTTTTATCCTCGCCTTTGGTGAAACGCAGCTGACTGACGAACAATGTCAGCAACT TGATGCGCTATTCAGCACGCCCGTCTGGATAAGCCAGGCGCAAGGCATCCGTGCTGGCCCTA 
TGTCCGGTAAAATGACTGGTATCGTAAAATGGTTCAACGCTGACAAAGGCTTCGGCTTCATCAC TCCT

$\underline{\text { HemK AP }-56}$

ATTAATACGACTCACTATAGGGGAATTGTGAGCGGATAACAATTCCCCTCTAGAAATAATTTTGT TTAACTTTAAGAAGGAGATATACATATGGAATATCAACACTGGTTACGTGAAGCAATAAGCCAA CTTCAGGCGAGCGAAAGCCCGCGGCGTGATGCTGAAATCCTGCTGGAACATGTTACCGGCAA AGGGCGTACTTTTATCCTCGCCTTTGGTGAAACGCAGCTGACTGACGAACAATGTCAGCAACT TGATTTCAGCACGCCCGTCTGGATAAGCCAGGCGCAAGGCATCCGTGCTGGCCCTATGTCCG GTAAAATGACTGGTATCGTAAAATGGTTCAACGCTGACAAAGGCTTCGGCTTCATCACTCCT

HemK AP - 54

ATTAATACGACTCACTATAGGGGAATTGTGAGCGGATAACAATTCCCCTCTAGAAATAATTTTGT TTAACTTTAAGAAGGAGATATACATATGGAATATCAACACTGGTTACGTGAAGCAATAAGCCAA CTTCAGGCGAGCGAAAGCCCGCGGCGTGATGCTGAAATCCTGCTGGAACATGTTACCGGCAA AGGGCGTACTTTTATCCTCGCCTTTGGTGAAACGCAGCTGACTGACGAACAATGTCAGCAATT CAGCACGCCCGTCTGGATAAGCCAGGCGCAAGGCATCCGTGCTGGCCCTATGTCCGGTAAAA TGACTGGTATCGTAAAATGGTTCAACGCTGACAAAGGCTTCGGCTTCATCACTCCT

$\underline{\text { HemK AP }-52}$

ATTAATACGACTCACTATAGGGGAATTGTGAGCGGATAACAATTCCCCTCTAGAAATAATTTTGT TTAACTTTAAGAAGGAGATATACATATGGAATATCAACACTGGTTACGTGAAGCAATAAGCCAA CTTCAGGCGAGCGAAAGCCCGCGGCGTGATGCTGAAATCCTGCTGGAACATGTTACCGGCAA AGGGCGTACTTTTATCCTCGCCTTTGGTGAAACGCAGCTGACTGACGAACAATGTTTCAGCAC GCCCGTCTGGATAAGCCAGGCGCAAGGCATCCGTGCTGGCCCTATGTCCGGTAAAATGACTG GTATCGTAAAATGGTTCAACGCTGACAAAGGCTTCGGCTTCATCACTCCT

HemK AP - 50

ATTAATACGACTCACTATAGGGGAATTGTGAGCGGATAACAATTCCCCTCTAGAAATAATTTTGT TTAACTTTAAGAAGGAGATATACATATGGAATATCAACACTGGTTACGTGAAGCAATAAGCCAA CTTCAGGCGAGCGAAAGCCCGCGGCGTGATGCTGAAATCCTGCTGGAACATGTTACCGGCAA AGGGCGTACTTTTATCCTCGCCTTTGGTGAAACGCAGCTGACTGACGAATTCAGCACGCCCGT CTGGATAAGCCAGGCGCAAGGCATCCGTGCTGGCCCTATGTCCGGTAAAATGACTGGTATCGT AAAATGGTTCAACGCTGACAAAGGCTTCGGCTTCATCACTCCT

HemK AP - 48

ATTAATACGACTCACTATAGGGGAATTGTGAGCGGATAACAATTCCCCTCTAGAAATAATTTTGT TTAACTTTAAGAAGGAGATATACATATGGAATATCAACACTGGTTACGTGAAGCAATAAGCCAA 
CTTCAGGCGAGCGAAAGCCCGCGGCGTGATGCTGAAATCCTGCTGGAACATGTTACCGGCAA AGGGCGTACTTTTATCCTCGCCTTTGGTGAAACGCAGCTGACTTTCAGCACGCCCGTCTGGAT AAGCCAGGCGCAAGGCATCCGTGCTGGCCCTATGTCCGGTAAAATGACTGGTATCGTAAAATG GTTCAACGCTGACAAAGGCTTCGGCTTCATCACTCCT

$\underline{\text { HemK AP }-46}$

ATTAATACGACTCACTATAGGGGAATTGTGAGCGGATAACAATTCCCCTCTAGAAATAATTTTGT TTAACTTTAAGAAGGAGATATACATATGGAATATCAACACTGGTTACGTGAAGCAATAAGCCAA CTTCAGGCGAGCGAAAGCCCGCGGCGTGATGCTGAAATCCTGCTGGAACATGTTACCGGCAA AGGGCGTACTTTTATCCTCGCCTTTGGTGAAACGCAGTTCAGCACGCCCGTCTGGATAAGCCA GGCGCAAGGCATCCGTGCTGGCCCTATGTCCGGTAAAATGACTGGTATCGTAAAATGGTTCAA CGCTGACAAAGGCTTCGGCTTCATCACTCCT

HemK AP - 44

ATTAATACGACTCACTATAGGGGAATTGTGAGCGGATAACAATTCCCCTCTAGAAATAATTTTGT TTAACTTTAAGAAGGAGATATACATATGGAATATCAACACTGGTTACGTGAAGCAATAAGCCAA CTTCAGGCGAGCGAAAGCCCGCGGCGTGATGCTGAAATCCTGCTGGAACATGTTACCGGCAA AGGGCGTACTTTTATCCTCGCCTTTGGTGAATTCAGCACGCCCGTCTGGATAAGCCAGGCGCA AGGCATCCGTGCTGGCCCTATGTCCGGTAAAATGACTGGTATCGTAAAATGGTTCAACGCTGA CAAAGGCTTCGGCTTCATCACTCCT

HemK AP - 42

ATTAATACGACTCACTATAGGGGAATTGTGAGCGGATAACAATTCCCCTCTAGAAATAATTTTGT TTAACTTTAAGAAGGAGATATACATATGGAATATCAACACTGGTTACGTGAAGCAATAAGCCAA CTTCAGGCGAGCGAAAGCCCGCGGCGTGATGCTGAAATCCTGCTGGAACATGTTACCGGCAA AGGGCGTACTTTTATCCTCGCCTTTTTCAGCACGCCCGTCTGGATAAGCCAGGCGCAAGGCAT CCGTGCTGGCCCTATGTCCGGTAAAATGACTGGTATCGTAAAATGGTTCAACGCTGACAAAGG CTTCGGCTTCATCACTCCT

$\underline{\text { HemK AP }-40}$

ATTAATACGACTCACTATAGGGGAATTGTGAGCGGATAACAATTCCCCTCTAGAAATAATTTTGT TTAACTTTAAGAAGGAGATATACATATGGAATATCAACACTGGTTACGTGAAGCAATAAGCCAA CTTCAGGCGAGCGAAAGCCCGCGGCGTGATGCTGAAATCCTGCTGGAACATGTTACCGGCAA AGGGCGTACTTTTATCCTCTTCAGCACGCCCGTCTGGATAAGCCAGGCGCAAGGCATCCGTGC TGGCCCTATGTCCGGTAAAATGACTGGTATCGTAAAATGGTTCAACGCTGACAAAGGCTTCGG CTTCATCACTCCT 
HemK AP - 38

ATTAATACGACTCACTATAGGGGAATTGTGAGCGGATAACAATTCCCCTCTAGAAATAATTTTGT TTAACTTTAAGAAGGAGATATACATATGGAATATCAACACTGGTTACGTGAAGCAATAAGCCAA CTTCAGGCGAGCGAAAGCCCGCGGCGTGATGCTGAAATCCTGCTGGAACATGTTACCGGCAA AGGGCGTACTTTTTTCAGCACGCCCGTCTGGATAAGCCAGGCGCAAGGCATCCGTGCTGGCC CTATGTCCGGTAAAATGACTGGTATCGTAAAATGGTTCAACGCTGACAAAGGCTTCGGCTTCAT CACTCCT

$\underline{\text { HemK AP }-36}$

ATTAATACGACTCACTATAGGGGAATTGTGAGCGGATAACAATTCCCCTCTAGAAATAATTTTGT TTAACTTTAAGAAGGAGATATACATATGGAATATCAACACTGGTTACGTGAAGCAATAAGCCAA CTTCAGGCGAGCGAAAGCCCGCGGCGTGATGCTGAAATCCTGCTGGAACATGTTACCGGCAA AGGGCGTTTCAGCACGCCCGTCTGGATAAGCCAGGCGCAAGGCATCCGTGCTGGCCCTATGT CCGGTAAAATGACTGGTATCGTAAAATGGTTCAACGCTGACAAAGGCTTCGGCTTCATCACTCC $\mathrm{T}$

HemK AP - 34

ATTAATACGACTCACTATAGGGGAATTGTGAGCGGATAACAATTCCCCTCTAGAAATAATTTTGT TTAACTTTAAGAAGGAGATATACATATGGAATATCAACACTGGTTACGTGAAGCAATAAGCCAA CTTCAGGCGAGCGAAAGCCCGCGGCGTGATGCTGAAATCCTGCTGGAACATGTTACCGGCAA ATTCAGCACGCCCGTCTGGATAAGCCAGGCGCAAGGCATCCGTGCTGGCCCTATGTCCGGTA AAATGACTGGTATCGTAAAATGGTTCAACGCTGACAAAGGCTTCGGCTTCATCACTCCT

HemK AP - 32

ATTAATACGACTCACTATAGGGGAATTGTGAGCGGATAACAATTCCCCTCTAGAAATAATTTTGT TTAACTTTAAGAAGGAGATATACATATGGAATATCAACACTGGTTACGTGAAGCAATAAGCCAA CTTCAGGCGAGCGAAAGCCCGCGGCGTGATGCTGAAATCCTGCTGGAACATGTTACCTTCAG CACGCCCGTCTGGATAAGCCAGGCGCAAGGCATCCGTGCTGGCCCTATGTCCGGTAAAATGA CTGGTATCGTAAAATGGTTCAACGCTGACAAAGGCTTCGGCTTCATCACTCCT

HemK AP - 30

ATTAATACGACTCACTATAGGGGAATTGTGAGCGGATAACAATTCCCCTCTAGAAATAATTTTGT TTAACTTTAAGAAGGAGATATACATATGGAATATCAACACTGGTTACGTGAAGCAATAAGCCAA CTTCAGGCGAGCGAAAGCCCGCGGCGTGATGCTGAAATCCTGCTGGAACATTTCAGCACGCC CGTCTGGATAAGCCAGGCGCAAGGCATCCGTGCTGGCCCTATGTCCGGTAAAATGACTGGTAT CGTAAAATGGTTCAACGCTGACAAAGGCTTCGGCTTCATCACTCCT 
HemK AP - 28

ATTAATACGACTCACTATAGGGGAATTGTGAGCGGATAACAATTCCCCTCTAGAAATAATTTTGT TTAACTTTAAGAAGGAGATATACATATGGAATATCAACACTGGTTACGTGAAGCAATAAGCCAA CTTCAGGCGAGCGAAAGCCCGCGGCGTGATGCTGAAATCCTGCTGTTCAGCACGCCCGTCTG GATAAGCCAGGCGCAAGGCATCCGTGCTGGCCCTATGTCCGGTAAAATGACTGGTATCGTAAA ATGGTTCAACGCTGACAAAGGCTTCGGCTTCATCACTCCT

$\underline{\text { HemK AP }-26}$

ATTAATACGACTCACTATAGGGGAATTGTGAGCGGATAACAATTCCCCTCTAGAAATAATTTTGT TTAACTTTAAGAAGGAGATATACATATGGAATATCAACACTGGTTACGTGAAGCAATAAGCCAA CTTCAGGCGAGCGAAAGCCCGCGGCGTGATGCTGAAATCTTCAGCACGCCCGTCTGGATAAG CCAGGCGCAAGGCATCCGTGCTGGCCCTATGTCCGGTAAAATGACTGGTATCGTAAAATGGTT CAACGCTGACAAAGGCTTCGGCTTCATCACTCCT

$\underline{\text { HemK AP }-24}$

ATTAATACGACTCACTATAGGGGAATTGTGAGCGGATAACAATTCCCCTCTAGAAATAATTTTGT TTAACTTTAAGAAGGAGATATACATATGGAATATCAACACTGGTTACGTGAAGCAATAAGCCAA CTTCAGGCGAGCGAAAGCCCGCGGCGTGATGCTTTCAGCACGCCCGTCTGGATAAGCCAGGC GCAAGGCATCCGTGCTGGCCCTATGTCCGGTAAAATGACTGGTATCGTAAAATGGTTCAACGC TGACAAAGGCTTCGGCTTCATCACTCCT

HemK AP - 22

ATTAATACGACTCACTATAGGGGAATTGTGAGCGGATAACAATTCCCCTCTAGAAATAATTTTGT TTAACTTTAAGAAGGAGATATACATATGGAATATCAACACTGGTTACGTGAAGCAATAAGCCAA CTTCAGGCGAGCGAAAGCCCGCGGCGTTTCAGCACGCCCGTCTGGATAAGCCAGGCGCAAG GCATCCGTGCTGGCCCTATGTCCGGTAAAATGACTGGTATCGTAAAATGGTTCAACGCTGACA AAGGCTTCGGCTTCATCACTCCT 


\section{Acknowledgements}

No person is an island and no work is done in isolation, so I would like to specifically thank a few of the people without whom I would not have been able to complete this work.

First, thank you to Prof. Marina Rodnina, for your guidance and advice throughout my time in this lab. Through thoughtful discussions, you have taught me the importance of attention to detail, merit in trying out many strategies for solving a problem, and how most protocols require some tinkering, while others are just set in stone.

I would also like to thank Dr. Ekaterina Samatova. I appreciate your perfectly considerate and timely motivational gestures throughout the highs and lows of this project. I enjoyed our regular critical discussion and data interpretation sessions and I benefited greatly from your valuable experimental advice.

To every person in the department of Physical Biochemistry past and present, thank you for booking the equipment diligently, generously sharing reagents and research tips and always being open and flexible to accommodate each other's experiments. It was splendid to conduct my $\mathrm{PhD}$ among conscientious and attentive colleagues. Special thank you goes out to Wolf Holtkamp, who at the very beginning of my $\mathrm{PhD}$ taught me that when a bench crisis arrives the only way is not to panic and "just proceed", I continue to benefit from this advice.

I am grateful for every bit of support and every opportunity that I have received from Steffen Burckhardt and the Molecular Biology office. To be in the Molbio program has been an extremely enriching experience and I feel grateful having been able to conduct my PhD work while being a part of it.

I would like to thank all my friends ----- for memes and laughs and holiday trips and stories and witness proclamations of life outside of the quite singular academic environment. And to my gang of ride-or-dies - Franzi, Madhobi and Cathie - I am grateful that you *knew* and I did not have to explain the trials and tribulations. Thank you for every lunch, brunch, dinner, drinks, stroll and hike, and every accompanying conversation, it was indispensable.

I would never have been able to reach this PhD milestone without the support of my family. Ačiū, Jorūne ir Raimondai, Morta, Kostai, diedukai ir Vlada, už patikimą ir nesvyruojanti palaikymą ir nuolatinį, energingą rūpinimąsi mano būviu ir sèkme. Jūsų dèka kiekvienos atostogos namuose prisuko mano valios ir energijos spyruoklę stengtis labiau ir tęsti toliau.

Finally, Dylan and Carling, your classes were a wonderful exercise in practicing to be present and learning to let go. 
